\title{
Diet, postprandial metabolism and endothelial function : effects of lutein-enriched eggs and trans- resveratrol
}

\author{
Citation for published version (APA):
}

van der Made, S. M. (2015). Diet, postprandial metabolism and endothelial function : effects of luteinenriched eggs and trans-resveratrol. [Doctoral Thesis, Maastricht University]. Maastricht University. https://doi.org/10.26481/dis.20150612sm

Document status and date:

Published: 01/01/2015

DOI:

10.26481/dis.20150612sm

Document Version:

Publisher's PDF, also known as Version of record

\section{Please check the document version of this publication:}

- A submitted manuscript is the version of the article upon submission and before peer-review. There can be important differences between the submitted version and the official published version of record. People interested in the research are advised to contact the author for the final version of the publication, or visit the DOI to the publisher's website.

- The final author version and the galley proof are versions of the publication after peer review.

- The final published version features the final layout of the paper including the volume, issue and page numbers.

Link to publication

\footnotetext{
General rights rights.

- You may freely distribute the URL identifying the publication in the public portal. please follow below link for the End User Agreement:

www.umlib.nl/taverne-license

Take down policy

If you believe that this document breaches copyright please contact us at:

repository@maastrichtuniversity.nl

providing details and we will investigate your claim.
}

Copyright and moral rights for the publications made accessible in the public portal are retained by the authors and/or other copyright owners and it is a condition of accessing publications that users recognise and abide by the legal requirements associated with these

- Users may download and print one copy of any publication from the public portal for the purpose of private study or research.

- You may not further distribute the material or use it for any profit-making activity or commercial gain

If the publication is distributed under the terms of Article $25 \mathrm{fa}$ of the Dutch Copyright Act, indicated by the "Taverne" license above, 
Diet, postprandial metabolism and endothelial function: effects of lutein-enriched eggs and trans-resveratrol 
The studies presented in this thesis were performed within NUTRIM School of Nutrition and Translational Research in Metabolism, which participates in the Graduate School VLAG (Food Technology, Agrobiotechnology, Nutrition and Health Sciences), accredited by the Royal Netherlands Academy of Arts and Sciences.

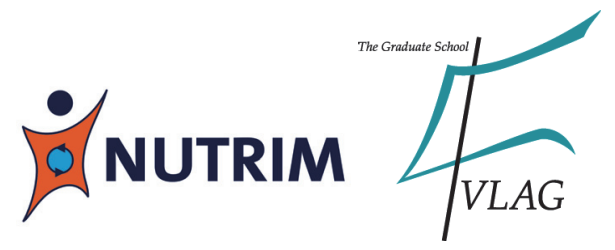

Financial support by DSM Nutritional Products and Newtricious R\&D for the publication of this thesis is gratefully acknowledged.
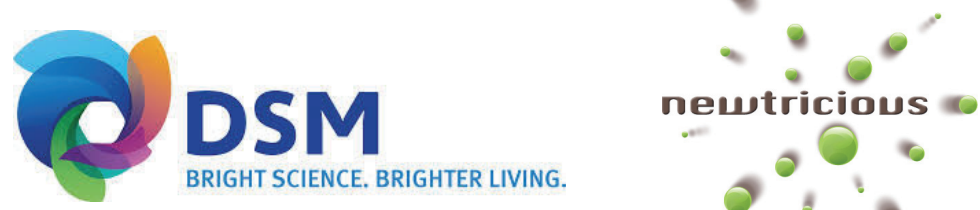

BRIGHT SCIENCE. BRIGHTER LIVING.

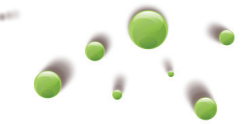

(C) Sanne Maria van der Made, 's Hertogenbosch 2015

Cover design: Proefschriftmaken.nl || Uitgeverij BOXPress

Layout: Sanne van der Made

Printed by: Proefschriftmaken.nl || Uitgeverij BOXPress

ISBN 9789462951709 


\title{
Diet, postprandial metabolism and
}

\author{
endothelial function: effects of \\ lutein-enriched eggs and \\ trans-resveratrol
}

\author{
PROEFSCHRIFT \\ ter verkrijging van de graad van doctor \\ aan de Universiteit Maastricht, \\ op gezag van de Rector Magnificus, Prof. dr. L.L.G. Soete \\ volgens het besluit van het College van Decanen, \\ in het openbaar te verdedigen \\ op vrijdag 12 juni 2015 om 14.00 uur
}

door

Sanne Maria van der Made

geboren te Tilburg op 6 januari 1986 


\section{Promotores}

Prof. dr. J. Plat

Prof. dr. ir. R.P. Mensink

\section{Beoordelingscommissie}

Prof. dr. P. Schrauwen (voorzitter)

Prof. dr. A. Bast

Dr. R. Draijer (Unilever R\&D, Vlaardingen, The Netherlands)

Dr. J.M. Geleijnse (Wageningen UR, Wageningen, The Netherlands)

Prof. dr. C.G. Schalkwijk 


\section{CONTENTS}

$\begin{array}{lll}\text { Chapter } 1 & \text { General Introduction } & 7\end{array}$

Chapter 2 Dyslipidemias: Pathophysiology, Evaluation and Management - 19 Polyphenols

Chapter 3 Resveratrol does not influence metabolic risk markers related to cardiovascular health in overweight and slightly obese subjects: a randomized, placebo-controlled crossover trial

Chapter $4 \quad$ Resveratrol does not affect markers for endothelial function and arterial flexibility in the fasting or postprandial phase: a randomized, placebo-controlled crossover trial

Chapter 5 Consuming a buttermilk drink containing lutein-enriched egg-yolk daily for 1 year increases plasma lutein but does not affect serum lipid or lipoprotein concentrations in adults with early signs of agerelated macular degeneration

Chapter 6 Daily consumption of a buttermilk drink containing lutein-enriched egg-yolks does not affect on postprandial metabolism or endothelial function

Chapter 7 General Discussion

References

Summary

Samenvatting

Valorisation

Dankwoord

List of publications

Curriculum vitae 



\section{CHAPTER 1}

General Introduction 


\section{Introduction}

The metabolic syndrome (MetS) includes a set of abnormalities in metabolic risk markers that increases the risk for developing cardiovascular disease (CVD) and type 2 diabetes mellitus (T2DM). For many years, several definitions for the MetS made by different societies, existed side-by-side (1-4). These definitions were largely overlapping, and ultimately, in 2009, a joint interim statement was presented by the International Diabetes Federation Task Force on Epidemiology and Prevention, the National Heart, Lung, and Blood Institute, the American Heart Association, the World Heart Federation, the International Atherosclerosis Society and the International Association for the Study of Obesity. In this statement, it was agreed that the presence of three out of five of the criteria given in Table 1.1 constitutes a diagnosis with the MetS $(5,6)$. The main risk factors for the MetS can be clustered into five themes, which are atherogenic dyslipidemia, vascular dysfunction, vascular inflammation, elevated plasma glucose and a prothrombotic state, which all have their own characteristics (Figure 1.1, based on (7)).

Table 1.1 Criteria for clinical diagnosis of the Metabolic Syndrome

\begin{tabular}{ll}
\hline Measure & Threshold for metabolic syndrome \\
\hline Elevated waist circumference & Population- and country-specific definitions exist. \\
& Here, the cut-off points for Caucasians are given. \\
Men & $\geq 94 \mathrm{~cm}^{1}$ \\
& $\geq 102 \mathrm{~cm}^{2}$ \\
& $\geq 80 \mathrm{~cm}^{1}$ \\
Women & $\geq 88 \mathrm{~cm}^{2}$ \\
& \\
Atherogenic dyslipidemia & \\
Elevated triglycerides & \\
Reduced HDL cholesterol & $<1.7 \mathrm{mmol} / \mathrm{L}$ \\
Men & $<1.3 \mathrm{mmol} / \mathrm{L}$ \\
Women & $\geq 130 \mathrm{mmHg}$ \\
Elevated blood pressure & \\
Systolic & $\geq 85 \mathrm{mmHg}$ \\
Diastolic & $\geq 5.6 \mathrm{mmol} / \mathrm{L}$ \\
Elevated fasting blood glucose &
\end{tabular}




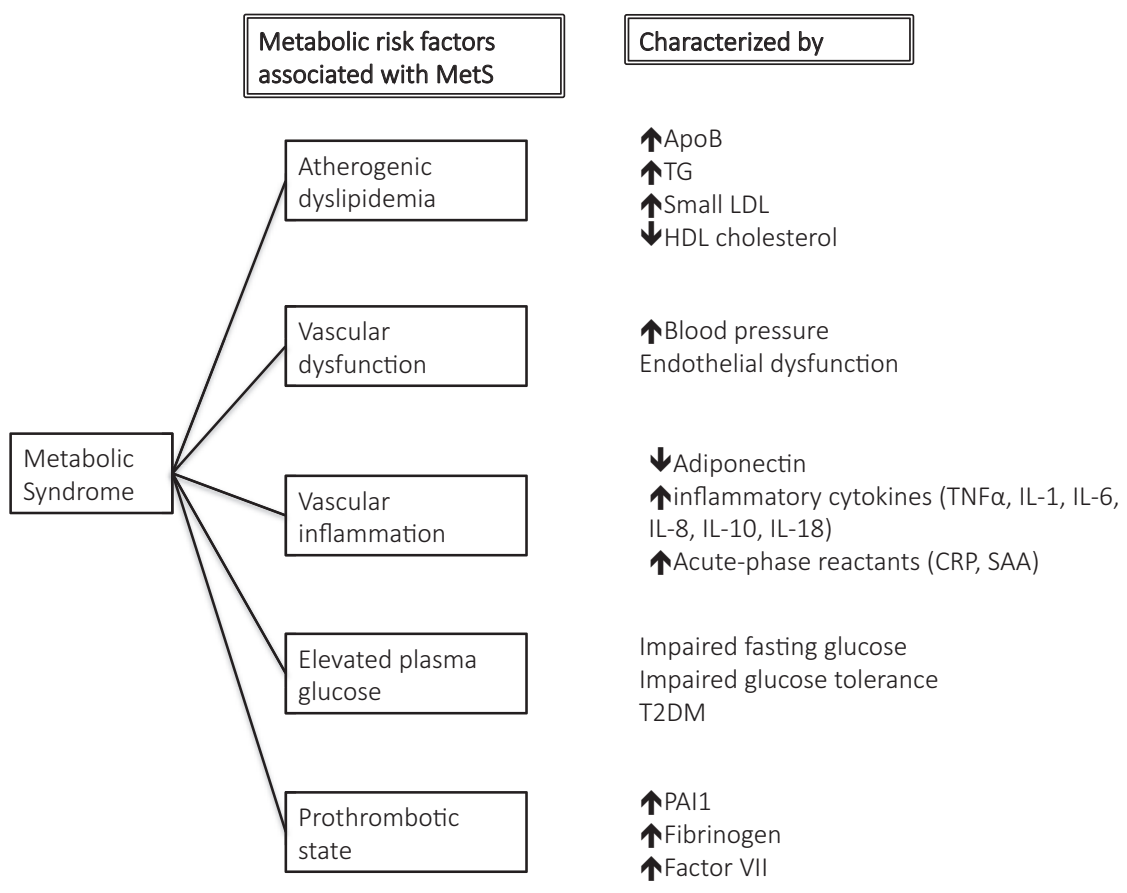

Figure 1.1 Main risk factors for the metabolic syndrome and the clinical manifestations that characterize these risk factors. ApoB; apolipoprotein B, TG; triglycerides, LDL; low-density lipoprotein, HDL; high-density lipoprotein, TNF $\alpha$; tumor-necrosis factor $\alpha$, IL; interleukin; CRP; C-reactive protein, SAA; serum amyloid A protein; T2DM, type 2 diabetes mellitus; PAI1; plasminogen-activator inhibitor 1 . Adapted from (7).

The prevalence of the MetS depends on the definition used and the population studied and varies worldwide between $10 \%$ and $40 \%$ (8). In The Netherlands, the prevalence is estimated to be $18 \%$ for men and $11 \%$ for women and increases with age (9). The relative risk for developing cardiovascular disease is doubled in persons suffering from the MetS, compared to those without the MetS, but is obviously dependent on the metabolic risk factors that are present (7).

\section{Cardiovascular disease}

Cardiovascular disease is the most common cause of mortality in high-income countries $(10,11)$, accounting for $35 \%$ of all deaths in Europe (12).

Atherosclerosis is the process underlying cardiovascular disease. All risk factors associated with the MetS play a role in atherosclerotic plaque development. Endothelial dysfunction is a very early event in atherosclerotic plaque development, which is a 
consequence of progressing atherogenesis, and occurs even before structural changes to the vasculature are apparent. Therefore, in terms of preventive interventions and treatments, identifying the presence of endothelial dysfunction is of utmost importance, because reverting the process of atherosclerotic plaque formation is still possible in the first decades of plaque development. All other major risk factors associated with the MetS play a role in atherosclerosis and they are also associated with the presence of endothelial dysfunction.

\section{Atherosclerotic plaque development}

According to the 'response to injury' hypothesis (13), endothelial dysfunction precedes the process of atherosclerotic plaque development. Atherosclerotic plaques develop when the endothelial cell layer of an artery is injured, also referred to as endothelial dysfunction, which can be caused by, for example, modified low-density lipoproteins (LDL), hypertension, diabetes mellitus, and free radicals formed through smoking. Endothelial dysfunction is present when this process continues uninterruptedly, leading to increased adhesiveness of the endothelium to leukocytes and platelets and increased endothelial permeability. Furthermore, the expression of leukocyte adhesion molecules such as L-selectin, integrins and platelet-endothelial-cell adhesion molecule-1 (PECAM-1) and endothelial adhesion molecules such as E-selectin, P-selectin, intercellular adhesion molecule-1 (ICAM-1) and vascular cell adhesion molecule-1 (VCAM-1) is upregulated. Additionally, the endothelium changes from anticoagulant to pro-coagulant and forms vasoactive molecules, cytokines and growth factors. Monocyte chemotactic protein and interleukin-8 (IL-8) migrate into the vascular wall, which is mediated by oxidized lowdensity lipoproteins. When the inflammatory process is not able to fight the cause of endothelial dysfunction, this process will proceed. This will lead to the development of fatty streaks, which consist of foam cells (lipid-laden monocytes and macrophages) and T-lymphocytes. Also, migration and proliferation of smooth muscle cells to the area of inflammation follows, where an intermediate lesion is formed that thickens the artery wall. In this stadium, the artery is able to compensate for the altered lumen by dilation (13). When this process continues, increased numbers of macrophages and lymphocytes enter the lesion, where they multiply, followed by a release of hydrolytic enzymes, cytokines and growth factors. In this way, the lesion keeps on growing, and ultimately an advanced, complicated lesion with a core of lipid and necrotic tissue is formed, which is covered by a fibrous cap. Finally, rupture or thinning of the fibrous cap can lead to thrombosis or restriction of the blood flow caused by narrowing of the lumen, ultimately leading to clinical manifestations such as myocardial or cerebral infarctions. 


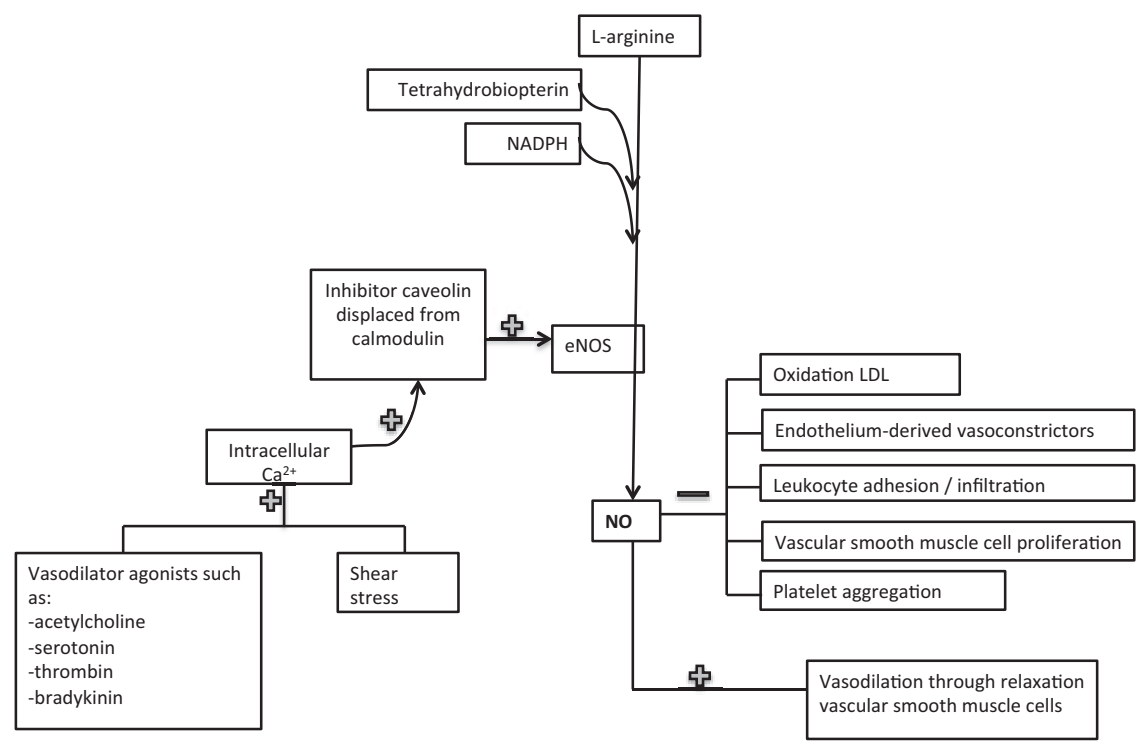

Figure 1.2 NO plays a key role in maintaining normal endothelial function. NO is produced by endothelial cells through the action of endothelial nitric oxide synthase (eNOS) on L-arginine, which requires cofactors such as tetrahydrobiopterin and nicotinamide adenine dinucleotide phosphate (NADPH). Vasodilator agonists or shear stress lead to increased intracellular Ca2+ concentrations, which in turn lead to displacement of caveolin form calmodulin and activation of eNOS. NO then diffuses to vascular smooth muscle cells, where it causes relaxation by activating guanylate cyclase and increased intracellular cyclic guanosine monophosphate concentrations. Based on (14).

\section{Endothelial (dys)function}

As indicated, vascular dysfunction represents one of the main risk factors for the MetS and endothelial dysfunction is a very early event in the cascade towards atherosclerotic plaque development. Endothelial dysfunction occurs when the balance between vasodilation and vasoconstriction in the vasculature is disturbed and is considered an early marker for atherosclerosis, even preceding angiographic or ultrasonic evidence of atherosclerotic plaque development $(14,15)$. The process of endothelial dysfunction originates primarily from a defective nitric oxide (NO) production or -activity (14). Through its effect on vasodilation, NO plays a key role in maintaining vascular homeostasis, as shown in figure 1.2. NO inhibits platelet adherence and aggregation, leukocyte infiltration into the vascular wall and proliferation of vascular smooth muscle cells and prevents the formation of oxidized low-density lipoproteins (16). This shows 
that NO production is a key mechanism in endothelial (dys)function and an important contributor to atherosclerotic plaque development (14).

In humans, endothelial function is non-invasively measured by flow-mediated dilation (FMD) (17). During an FMD measurement, a high-resolution ultrasound recording of the brachialis artery is made, while the blood flow to the extremities is impeded by inflating a cuff, positioned on the underarm, to suprasystolic pressure. When the cuff is released, hyperemic flow and shear stress are induced, which provokes the endothelium to release NO, subsequently leading to vasodilation. The degree of vasodilation is expressed as the percentage increase in brachial artery diameter from baseline to maximal dilation (FMD\%). Next to its non-invasive character, measurement of ultrasound-based FMD is relatively repeatable and reproducible and is therefore useful in study designs with repeated measurements to study disease reversibility. Also, FMD reflects important vascular physiology as discussed above. Disadvantages of this technique include that it is operator-dependent as it is technically demanding and requires a specific training. Subject preparation should be performed by specific guidelines, among which are being fasted for at least 8 to $12 \mathrm{~h}$ before the measurement and measurements should be performed in a quiet, temperature-controlled room. The measurement then should be performed according to a tight protocol with the appropriate, costly equipment. Moreover, analysis of brachial artery reactivity is highly dependent on the quality of the ultrasound images (17).

Several studies have shown positive effects of both nutritional and pharmacological interventions on FMD in subjects with either a history of vascular disease, hypertriglyceridemia or the metabolic syndrome. Supplementation with omega-3 fatty acids was found to lower TG concentrations and improve FMD in hyperlipidemic subjects and subjects with the metabolic syndrome $(18,19)$. Both lipid-lowering drugs and angiotensin converting enzyme (ACE) -inhibitors, widely known as blood pressure lowering agents, have extensively been shown to improve endothelial function $(20,21)$, which implies that an unfavorable lipid profile and -blood pressure are prerequisites for impaired FMD values.

\section{Arterial stiffness}

Increased central arterial stiffness is a hallmark of vascular aging that is recognized as a surrogate endpoint for CVD. Measuring arterial stiffness is increasingly used in the clinical setting, and associated with presence of CV risk factors and atherosclerotic disease (22). As shown in figure 1.3, several causes underlie arterial stiffness. Disturbing the balance between the content of the two major proteins compromising the vascular walls, collagen and elastin, is the main contributor to the development of vascular stiffness. Stimulation of an inflammatory milieu leads to dysregulation of this balance 
through overproduction of collagen. Increased arterial stiffness causes an increased cardiac afterload, which leads to an impaired coronary arterial blood supply, atherogenesis and microvascular damage (23). Breakdown of elastin fibers in arterial walls is one of the mechanisms that lead to increased arterial stiffness. Two other mechanisms resulting in increased arterial stiffness are damage to the endothelium and an increase in mean arterial pressure. During the course of a lifetime, the arterial tree stiffens, which leads to an increased propagation of the pressure wave, which in turn increases central pulse pressure, resulting in profound changes in the arterial pressure waveform. When arteries stiffen, the reflected wave arrives at the heart early during systole, caused by the high PWV. This leads to a decreased travel time of the reflected wave from the periphery, resulting in an increased reflection amplitude.

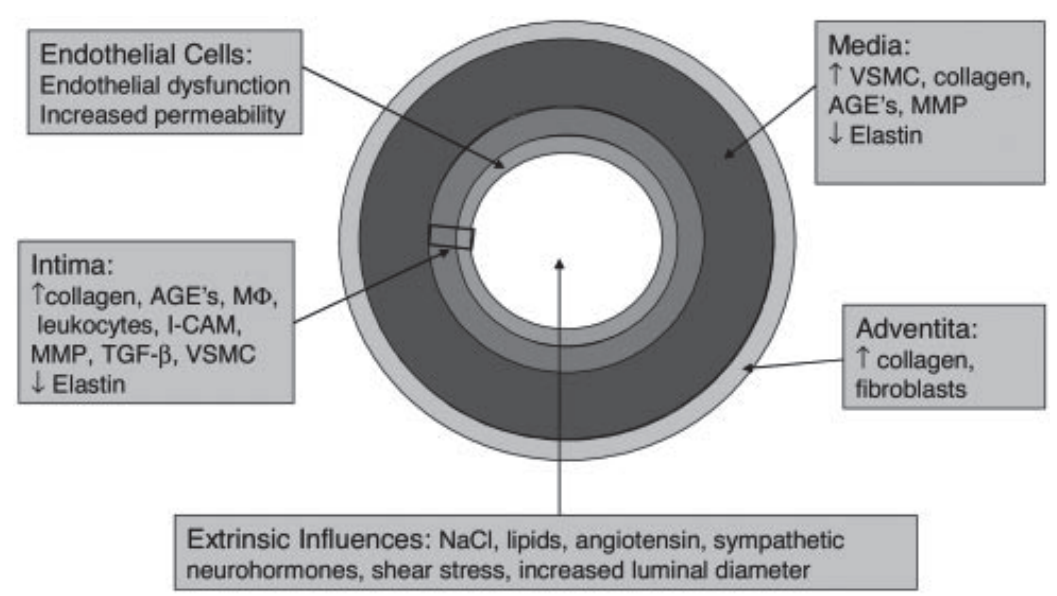

Figure 1.3 Summary of the multiple causes and locations of arterial stiffness. Adapted from (22).

PWV measurement is a direct method to determine arterial stiffness, generally accepted as the most simple, non-invasive, robust and reproducible method (24) and is defined as 'the speed of the pressure wave travelling along an arterial segment, determined by the length of the arterial segment under study divided by the time it takes for a pressure wave to travel from arterial location A to arterial location $B$ within this segment' (25). Carotid-femoral pulse wave velocity $\left(P W V_{c f}\right)$ is considered to be the gold standard in- and the most clinically relevant method to measure arterial stiffness. $P W V_{c f}$ is the most clinically relevant method to measure PWV, as it is measured along the aortic and aorto-iliac pathway, which is the path that is responsible for most of the pathophysiological effects of arterial stiffness, because the aorta and its first branches are directly connected to the left ventricle. $P W V_{c f}$ can be assessed through applying the 
principle of applanation tonometry at the femoral and carotid artery and direct computation of the time delay between the upstroke of the arterial pressure wave measured at these sites. Also, distance between both sites should be assessed accurately. This is one of the pitfalls of the PWV measurement, since it should be done in a standardized fashion as proposed recently (26). Other limitations of this measurement include the difficulty to record the femoral pressure waveform in subjects with the metabolic syndrome, obesity, diabetes, and peripheral artery disease (27).

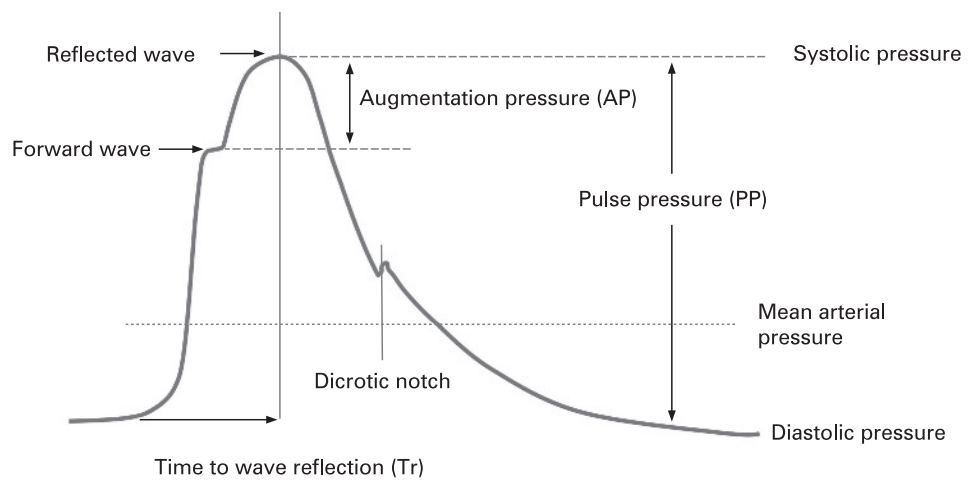

Figure 1.4 An aortic pulse waveform derived by applanation tonometry of the radial artery with the SphygmoCor device. Adapted from (28).

Arterial stiffness can also be assessed from parameters in the pressure waveform derived from pulse wave analysis (PWA) at the radial artery or femoral artery. Through a validated transfer function, the corresponding aortic pressure waveform can be generated from an averaged radial artery waveform (figure 1.4). Subsequently, augmentation index (Alx), and central blood pressure can be assessed. Alx is defined as 'the difference between the first and second peaks of the central arterial waveform, expressed as a percentage of the pulse pressure' (29) or 'the supplementary increase in blood pressure during systole due to the reflection of the forward travelling pressure waves from the peripheral circulation' (25) and provides indirect information on arterial stiffness as it is an estimate of stiffness and wave reflection. Augmentation index is calculated by the following formula:

$$
A \mid x=\frac{\left(P_{2}-P_{1}\right)}{P P} \times 100 \%
$$

In which $\mathrm{P}_{2}$ represents systolic pressure, $\mathrm{P}_{1}$ the systolic peak of the forward wave and PP the pulse pressure as depicted in Figure 1.4. 


\section{Atherogenic dyslipidemia}

Atherogenic dyslipidemia, characterized by increased LDL cholesterol- and TG concentrations and decreased HDL cholesterol concentrations, is one of the main risk factors of the MetS and plays an important role in endothelial dysfunction and CVD development.

Following meal intake, digestion of dietary fat starts in the stomach and small intestine. Short-chain fatty acids are absorbed passively and released directly from the intestinal capillaries into the portal circulation. In the intestine, long-chain and very long-chain fatty acids are actively absorbed, re-esterified into TG and incorporated into apolipoprotein B48-rich chylomicrons. Through the lymphatic system, TGs enter the circulation via the vena subclavia. After being hydrolyzed by lipoprotein lipase (LPL), the released free fatty acids (FFAs) function as an energy source for muscles or are stored in adipose tissue. Increased plasma TG concentrations for a prolonged time in the postprandial phase are considered to be pro-atherogenic, because high serum TG concentrations might lead to increased TG-rich lipoprotein remnants, such as small very low density lipoprotein (VLDL) and chylomicron remnants, which may enter the endothelium and increase plaque formation (30).

TG is also synthesized in the liver, where TG molecules are incorporated into VLDL particles for transport to the peripheral tissues. Again through the action of LPL, VLDL particles release TG in the form of FFAs to the tissues. This increases the density of the VLDL particle, which then becomes an intermediate-density lipoprotein (IDL). When IDL further releases its TG and apolipoprotein (except for apolipoprotein B100) content, it becomes an LDL particle. LDL particles are the main carriers of cholesterol to peripheral tissues, where they are internalized through the LDL receptor. High serum LDL cholesterol concentrations are recognized as a major risk factor for CVD. When LDL particles become trapped in an artery, they can be oxidized and subsequently internalized by macrophages. This process is followed by foam cell formation, through formation of lipid peroxides and accumulation of cholesterol esters. LDL further activates these foam cells when modified and taken up by macrophages. The inflammatory response is further expanded by LDL, because modified LDL is chemotactic for other monocytes, up-regulates macrophage colony-stimulating factor and monocyte chemotactic protein. In short, LDL particles that become trapped in the vascular endothelium play a crucial role in the development of atherosclerotic plaques.

Typically, HDL particles mediate the process of 'reversed cholesterol transport', in which the efflux of cholesterol from non-hepatic cells, such as macrophages, to the liver is described. Next, the liver excretes these sterols as such or metabolizes them into bile acids into the intestine for subsequent excretion via feces. HDL cholesterol is hypothesized to provide cardiovascular protection by promoting this process. Epidemiologic research showed evidence for the CVD risk protecting effect of HDL (3134). Evidence points towards increasing apolipoprotein A-I (apoA-I) concentrations as a 
more important target for reducing CVD risk (35). ApoA-I is the main protein constituent of HDL particles and plays a key role in many of the favorable effects ascribed to HDL particles, such as their anti-inflammatory, anti-thrombotic and anti-oxidative effects (36, 37). However, more recent clinical research showed no link between increasing HDL cholesterol concentrations through pharmacological interventions and clinical cardiovascular risk reduction (38-40). In this regard, measuring HDL function through cholesterol efflux capacity might be more valuable.

Insulin and glucose metabolism are associated with atherogenic dyslipidemia. Normally, insulin inhibits the production of apoB, which is the major apolipoprotein found on VLDL particles, as well as hepatic VLDL secretion into the circulation. However, in the insulin resistant state, hepatic TG synthesis, apoB production and VLDL secretion is increased, caused by an increased flux of FFA to the liver (41). This leads to an enhanced VLDL output by the liver and increased serum TG concentrations. Next to this, insulin stimulates LPL-activity, the major mediator in VLDL clearance. Therefore, in the insulin resistant state, not only hepatic VLDL production is increased but VLDL clearance is reduced, which also contributes to elevated serum TG concentrations (42). Next to this, insulin stimulates the release of vasodilator substances, such as NO and prostaglandins, from the vascular endothelium, where it thus plays an important role in maintaining vascular homeostasis (43).

\section{Strategy to measure changes in the metabolic profile}

Improving the metabolic profile through lifestyle interventions might be an obvious strategy to reduce arterial stiffness, improve endothelial function and the lipid profile, thereby lowering the risk for developing MetS. Lifestyle factors include reducing body weight, increasing the amount of exercise, improving the general dietary pattern according to the guidelines and by including nutritional compounds with an added effect on health in the diet via consumption of the so-called functional foods.

An increasingly used approach to assess the effect of nutrition on metabolic changes is providing a metabolic challenge. This challenge can be given in different ways, such as an oral glucose or fat load, exercise or vaccination and assesses metabolic changes in a sensitive and physiologic relevant way. A high fat meal for instance is shown to alter metabolism, increases inflammation and oxidative stress, and leads amongst others, to reduced vascular function $(44,45)$. In the studies described in this thesis, a dietary challenge is introduced, which is hypothesized to enable the possibility to measure subtle changes in the metabolic profile, introduced by specific interventions.

The interventions described in this thesis focus on the effects of longterm intake of dietary cholesterol (via lutein enriched egg yolks) and short-term intake of trans-resveratrol (via capsules) on markers for cardiovascular health. These two 
nutritional components were chosen to both describe a dietary compound that is widely discussed for its proposed negative effects on cardiovascular health -dietary cholesteroland to describe a compound -trans-resveratrol- that is widely studied for its proposed beneficial effects on metabolic health outcomes.

The main goal of the research presented in this thesis is to assess the effects of a functional food and a nutritional compound on metabolic indices related to CVD, with a special focus on postprandial metabolism and endothelial function.

\section{Outline of the thesis}

This thesis describes the results of two human dietary intervention trials, which were hypothesized to intervene with risk markers related to cardiovascular health. Chapter 2 discusses a specific group of nutritional compounds, namely polyphenols, regarding their effect on dyslipidemia. The main focus in this chapter is on flavonoids from cocoa, green tea catechins and the stilbene trans-resveratrol. Trans-resveratrol is studied in the human intervention trial that is described in the following chapters. Chapter 3 describes the effect of 4-wk trans-resveratrol intake on metabolic risk markers related to cardiovascular health, while chapter 4 focuses on its effect on endothelial function, arterial flexibility and postprandial metabolism. The next two chapters describe a human dietary intervention trial in which subjects were asked to take a lutein-enriched egg-yolk beverage daily for one year in order to study the effect on ophthalmic parameters related to macular degeneration. As this beverage contains cholesterol, the effect on lipid and lipoprotein metabolism (chapter 5) and endothelial function and postprandial metabolism (chapter 6) was studied and described in this thesis. Finally, in chapter 7 the main findings and conclusions of the studies described in this thesis are discussed and put into a broader perspective. 



\section{Dyslipidemias: Pathophysiology, Evaluation and Management - Polyphenols}




\begin{abstract}
In recent years, the effects of various polyphenols on cardiovascular risk markers have been examined. We here focus on flavonoids from cocoa, the stilbene trans-resveratrol, and green tea catechins. Short-term studies ( $<2$ weeks) suggested beneficial effects of flavanol-rich cocoa products or dark chocolate consumption on LDL cholesterol, but these effects were not confirmed in longer-term studies. Trans-resveratrol had no effects. Green tea catechins significantly lowered LDL cholesterol. One meta-analysis suggested beneficial, but small effects of chocolate and cocoa on HDL cholesterol. None of the polyphenols affected serum triacylglycerol. There is also evidence that chocolate, cocoa, and/or cocoa flavanols beneficially affect flow-mediated dilatation (FMD). Finally, attention is paid to the mechanisms underlying the effects of cocoa flavonoids and of trans-resveratrol on dyslipidemia management and endothelial function.
\end{abstract}




\section{Introduction}

Coronary heart disease (CHD) is still a major health problem in many countries. It is well known that an increased serum concentration of low-density lipoprotein (LDL) cholesterol is a powerful risk factor for CHD. Epidemiological studies also suggest that high serum concentrations of high-density lipoprotein (HDL) cholesterol may protect against CHD, although results from recent intervention studies with drugs do not support a causal relationship between HDL and CHD risk. Traditionally, reducing saturated and trans fatty acids intake has been the cornerstone in the management of dyslipidemia. However, in recent years, many other dietary components have attracted much interest. This has led, with different degrees of success, to the search and testing of specific foods and food components that may help to improve the serum lipoprotein profile. However, evidence is emerging that diet also affects other risk markers for CHD, such as endothelial function, blood pressure, inflammation, and platelet function.

This chapter will review the role of polyphenols in dyslipidemia management. In addition, the relation between polyphenols and endothelial function will be briefly addressed. Focus will be on flavonoids from cocoa and the stilbene trans-resveratrol, as flavonoids from cocoa have been extensively studied in the past, while recent studies have ascribed possible cardioprotective effects to trans-resveratrol. Metabolism of these polyphenols will be discussed as well.

\section{History}

Polyphenols are metabolites produced by higher plants and are important for pigmentation, reproduction, growth and protection against pathogens. It has for long been recognized that foods rich in polyphenols may possess healthy properties, such as anti-oxidative, antibacterial, antihypertensive and anti-inflammatory effects. In humans, the potential beneficial effect of polyphenols on cardiovascular disease has generated a great amount of scientific interest during the past decades. Hertog et al. were among the first to suggest a strong protective effect of flavonoids, a subgroup of polyphenols found in tea, onions and apples on coronary heart disease related deaths (46). The French Paradox, the observation that the incidence of CHD is low in the French population, despite a high dietary intake of saturated fat, also supported the notion that polyphenols may have beneficial effects on cardiovascular disease. This association has been attributed to increased intakes of resveratrol, a polyphenol found in foods such as red wine and grapes. This French paradox has formed the basis for numerous papers on the relation between polyphenol intake, and more specifically polyphenols from grapes, 
and cardiovascular health. Also studies in The Kuna Indians of the San Blas islands of Panama supported the idea that polyphenols may improve cardiovascular health. This population consumed on average three 10-ounce cups of cocoa beverage each day. The prevalence of hypertension among Kuna Indians was very low (2.2\%), blood pressure did not increase with age, and a lower rate of myocardial infarction and stroke was found as compared to mainland Panamanians (47). In another study, an inverse relation was found between cocoa intake and cardiovascular mortality in men aged 65 to 84 years from Zutphen, a city in the Netherlands (48). Men in the highest tertile of cocoa intake consumed on average $4.2 \mathrm{~g}$ cocoa daily, which is equal to a daily intake of $10 \mathrm{~g}$ dark chocolate, and had a 50\% lower risk than men in the lowest tertile, who did not consume cocoa at all. An inverse relation between cocoa intake and systolic and diastolic blood pressure was also found, but this was not the main explanation for the observed lower cardiovascular risk in this group. In another epidemiologic study in survivors (4570 years of age) of an acute myocardial infarction, an inverse association was found between chocolate consumption and cardiac mortality (49). These examples have certainly contributed to the immense growth of scientific interest in the relation between polyphenol intake, dyslipidemias, and cardiovascular health during the past decades.

\section{Chemical structure}

Each structure that includes several hydroxyl groups on aromatic rings can be defined as a polyphenol. Polyphenolic compounds can be divided into distinct groups based on the number of phenol rings and by the structural elements attached to these rings. In this way, four main polyphenol groups have been identified: (1) phenolic acids, (2) flavonoids, (3) stilbenes and (4) lignans (Figure 2.1). 
Phenolic acids -

Hydroxybenzoic acids<smiles>[R]c1cc(C(=O)O)cc([R3])c1[R2]</smiles>

Flavonoids Flavonols<smiles>[R3]c1cc(-c2oc3cc(O)cc(O)c3c(=O)c2O)cc([Y9])c1[R]</smiles>

Lignans -

Secoisolariciresinol<smiles>COc1cc(CC(CO)C(CO)Cc2ccc(O)c(OC)c2)ccc1O</smiles>

Figure 2.1 Chemical structure of the four main polyphenolic compounds. Hydroxybenzoic acids is drawn as an example of a subgroup of phenolic acids; the flavonols as the subgroup of the flavonoids; resveratrol belongs to the stilbenes and secoisolariciresinol is an example of the lignans.

Several products rich in these compounds and their estimated daily intakes are listed in Table 2.1 (50).

\section{Phenolic acids}

Phenolic acids can be classified into derivatives of benzoic acid or derivatives of cinnamic acid. Hydroxybenzoic acids are found in only a few plants eaten by humans. Caffeic acid is the major representative of a hydroxycinnamic acid and occurs in foods mainly as an ester with chlorogenic acid. Coffee consumers have an intake of 0.5-1g of chlorogenic acid. Hydroxycinnamic acid is also found in amounts varying from $0.5-2 \mathrm{~g} / \mathrm{kg}$ fresh weight, mainly in the skin of mature fruits. Wheat grain contains on average 0.8-2.0 g of ferulic acid -also a hydroxycinnamic acid- per kilogram dry weight. Phenolic acids are also present in rice, wheat, and oat flour in quantities of $70-90 \mathrm{mg} / \mathrm{kg}$ fresh weight, whereas corn flour may contain up to $300 \mathrm{mg} / \mathrm{kg}$ fresh weight. 


\section{Flavonoids}

The flavonoids are divided into six subclasses, depending on the oxidation status and saturation of the heterocycle group that is part of the flavonoid skeletal structure: (1) flavonols, (2) flavones, (3) isoflavones, (4) flavanones, (5) anthocyanidins and (6) flavanols. These subclasses share a common structure that consists of two aromatic rings, bound by three carbon atoms that form an oxygenated heterocycle. Flavonoids naturally occur mostly as glycosides rather than as aglycones.

\section{Flavonols}

The most widely known flavonols are quercetin and kaempferol. Flavonols mainly accumulate in the skin and leaves of vegetables, due to the fact that the biosynthesis of flavonols is stimulated by light. Therefore, the flavonol content of the same species can be very different; cherry tomatoes for instance have a higher flavonol content than regular tomatoes, caused by the different ratios of skin to whole fruit.

\section{Flavones}

Flavones are present in herbs such as parsley, but also chamomile tea, celery, tangerines, and some other citrus fruits contain flavones.

Table 2.1. Products rich in polyphenols and their estimated daily intakes.

\begin{tabular}{|c|c|c|c|}
\hline Main compound & Subgroup & Products rich in this compound & $\begin{array}{l}\text { Estimated daily } \\
\text { intake }(\mathrm{mg} / \mathrm{d})\end{array}$ \\
\hline \multirow[t]{2}{*}{ Phenolic acids } & Benzoic acids & Red fruits, black radish, onions, tealeaves & \\
\hline & Cinnamic acids & $\begin{array}{l}\text { Grains and seeds, coffee, apples, blueberries, } \\
\text { cherries, kiwis and plums }\end{array}$ & \\
\hline \multirow[t]{6}{*}{ Flavonoids } & Flavonols & $\begin{array}{l}\text { Tea, onions, curly kale, leek, broccoli, } \\
\text { tomatoes }\end{array}$ & 13 \\
\hline & Flavones & Parsley, chamomile tea, celery, tangerines & 1.6 \\
\hline & Isoflavones & Soybeans, soymilk, tofu, tempeh & $\begin{array}{l}\text { USA/Netherlands: } \\
1.2 \\
\text { Asia: } 25-50\end{array}$ \\
\hline & Flavanones & Grapefruits, oranges, lemons, tomatoes, mint & 14.4 \\
\hline & Anthocyanidins & $\begin{array}{l}\text { Cranberries, blackberries, eggplant, cabbage, } \\
\text { beans, onions, radishes }\end{array}$ & 3.1 \\
\hline & Flavanols & $\begin{array}{l}\text { Tea leaves, cocoa beans, dark chocolate, } \\
\text { apples, blueberries, grapes, apricots }\end{array}$ & 156 \\
\hline Stilbenes & & Red wine, black grapes & \\
\hline Lignans & & Cereals, berries, vegetables, flaxseed & \\
\hline
\end{tabular}




\section{Isoflavones}

Soybeans are a main source of isoflavones, whereas its content in other beans and peas like kidney beans, black beans and chickpeas is low. The level of the isoflavones genistein and daidzein in soybeans varies and depends on the geographic zone where the beans are cultivated. Growing conditions and processing also influences the isoflavone content of soybeans, which varies between $580-3800 \mathrm{mg} / \mathrm{kg}$ fresh weight. In soymilk, this content lies between $30-175 \mathrm{mg} / \mathrm{L}$. The intake of isoflavones differs widely around the world. In Asian countries, more soy products are consumed compared to Western countries, which is reflected in a higher estimated daily intake, as indicated in Table 2.1.

\section{Flavanone}

Flavanones are mainly found in the solid parts and the membranes separating the segments of citrus fruits. Therefore, a five times higher flavanone content is found in whole fruits compared to juice.

\section{Anthocyanidin}

Anthocyanidin is present in fruits and vegetables that have red, blue and purple pigments. Blackcurrants and blackberries contain about 2 - 4 g of anthocyanidin per $\mathrm{kg}$ fresh weight. The amount of anthocyanidins in a food product relates to its color intensity. Furthermore, anthocyanidin is found in fruit skin and its content becomes higher as a fruit ripens.

Flavanol / flavan-3-ol

The main flavanols are catechin and epicatechin, which are present in cocoa beans, dark chocolate and green tea. A cup of green tea can provide up to $200 \mathrm{mg}$ catechins. Several processes and conditions affect the flavanol content of cocoa, such as the variety and country of origin. Also the fermentation and roasting process, that has been applied, determines the flavanol content of cocoa beans.

\section{Stilbenes}

The most widely known and studied stilbene is trans-resveratrol, which is found in low quantities in red wine and in the skin of black grapes. The average trans-resveratrol content of red wines is $1.9 \mathrm{mg} / \mathrm{L}$, varying from non-detectable levels up to $14.3 \mathrm{mg} / \mathrm{L}$. Trans-resveratrol is thought to play a role in explaining the 'French-paradox'.

\section{Lignans}

A rich dietary source of lignans is flaxseed (>300 mg/100g). Other sources are cereals, grains, fruits and certain vegetables. Lignan content of grain products varies from 7 to $764 \mathrm{mg} / 100 \mathrm{~g}(51)$. 


\section{Effects of polyphenols on lipid and lipoprotein metabolism}

In the following paragraphs, effects of chocolate and cocoa on lipid and lipoprotein metabolism will be reviewed. Chocolate and cocoa are main sources of flavanols, such as epicatechin. In addition, attention will be paid to the stilbene resveratrol, a polyphenol with supposed cardioprotective effects. Finally, the effects of tea catechins and soy isoflavones will be discussed.

\section{Flavonoids}

In a recent meta-analysis, effects of flavanol-rich cocoa products or dark chocolate on the serum lipid profile were summarized (52). Ten controlled intervention studies were identified, including 320 subjects. Daily flavanol intake between the studies varied widely (88 - $963 \mathrm{mg})$. Compared with control, it was estimated that consumption of the cocoa products or dark chocolate for 2 weeks significantly decreased LDL cholesterol by -0.15 $\mathrm{mmol} / \mathrm{L}$ (95\% Cl: $-0.27,-0.03 \mathrm{mmol} / \mathrm{L})$. No significant effect on LDL cholesterol was observed in longer-term studies (4-12 weeks). Total cholesterol concentrations were significantly reduced by $0.16 \mathrm{mmol} / \mathrm{L}(95 \% \mathrm{Cl}$ : $-0.30,-0.02 \mathrm{mmol} / \mathrm{L})$. In both short term and longer-term studies, no significant effects on serum HDL cholesterol and triglyceride levels were observed (Figure 2.2).

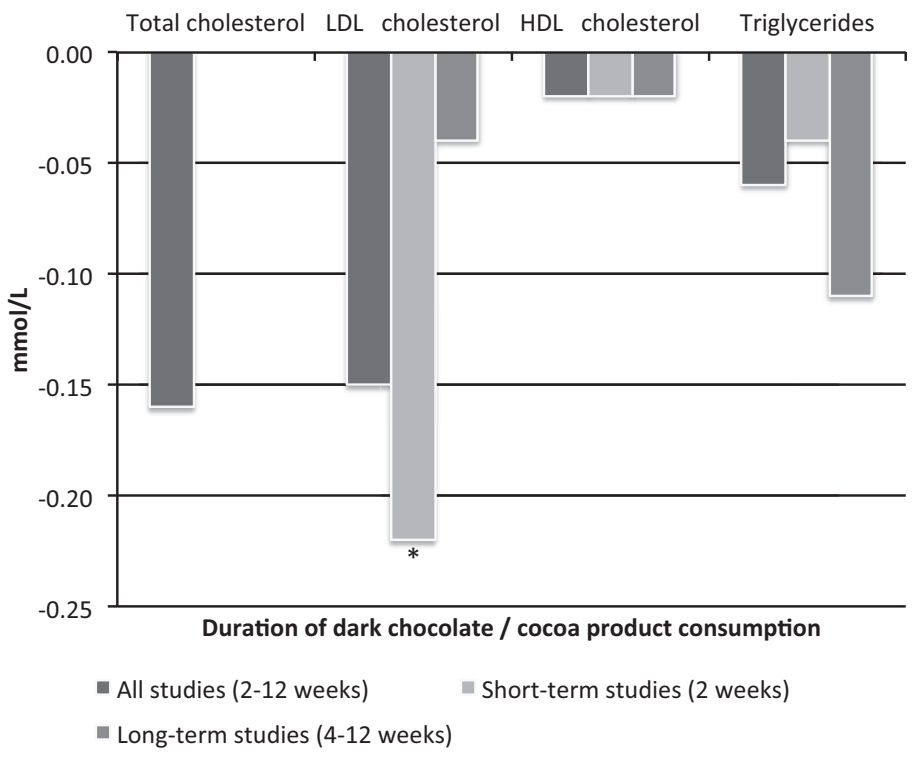

Figure 2.2. Effects of dark chocolate/cocoa product consumption on serum total cholesterol, LDL cholesterol, $\mathrm{HDL}$ cholesterol and triglyceride concentrations. A statistically significant $\left(^{*}\right)$ reduction in serum LDL cholesterol 
was found in short-term studies, while other lipids and lipoproteins did not change. In longer-term (4-12 weeks) studies, effects did not reach statistical significance. Effects of study duration on serum total cholesterol levels were not reported. (from Tokede, O.A., Gaziano, J.M., and Djoussé, L. Eur J Clin Nutr., 65, 879-886, 2011).

A possible dose-effect relationship was examined by dividing the studies based on the intake of flavanols (either $<500 \mathrm{mg}$ or $>500 \mathrm{mg}$ flavanol daily). Surprisingly, a daily flavanol intake of $<500 \mathrm{mg}$ appeared to lower LDL cholesterol more efficiently than intakes $\geq 500 \mathrm{mg}(-0.20 \mathrm{mmol} / \mathrm{L}$ vs. $-0.11 \mathrm{mmol} / \mathrm{L}$, respectively), but the differences between these effects were not statistically significant (Figure 2.3).

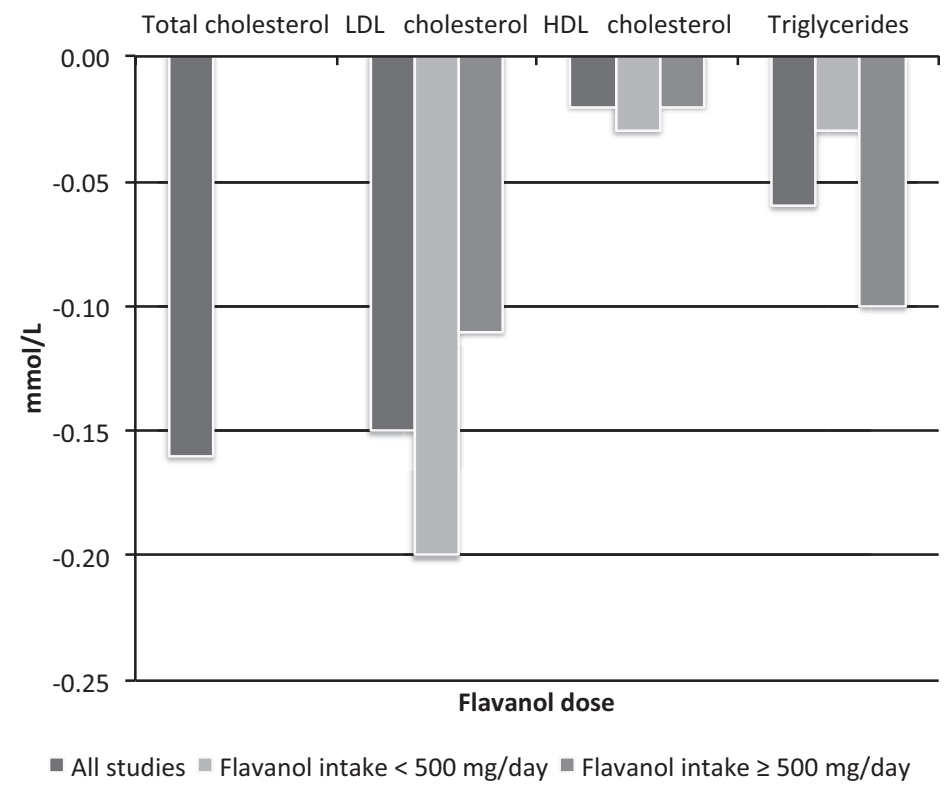

Figure 2.3. Effects of flavanol intake on serum total cholesterol, LDL cholesterol, HDL cholesterol and triglyceride concentrations. Differences in effects between the two levels of intakes did not reach statistical significance. Effects of different intake level on serum total cholesterol levels were not reported. (from Tokede, O.A., Gaziano, J.M., and Djoussé, L. Eur J Clin Nutr., 65, 879-886, 2011).

A comparable LDL-cholesterol lowering effect of $-0.07 \mathrm{mmol} / \mathrm{L}(95 \% \mathrm{Cl}:-0.14,0.00$ $\mathrm{mmol} / \mathrm{L}$ ) was found in a more recent meta-analysis that included 21 studies and 986 subjects (53). In studies that lasted maximally three weeks, a mean effect on serum LDL cholesterol of $-0.22 \mathrm{mmol} / \mathrm{L}$ was observed. However, no effect was observed in longerterm studies (3 to 26 weeks), which raises questions on the clinical usefulness of these observations. Without considering study duration, HDL cholesterol was increased by $0.03 \mathrm{mmol} / \mathrm{L}(95 \% \mathrm{Cl}: 0.00,0.06 \mathrm{mmol} / \mathrm{L})$. Beneficial effects on HDL cholesterol were more pronounced in longer-term trials (>3-26 wks) (Figure 2.4 ). In this meta-analysis, the 
effects of epicatechin (and not of total flavanols) were also examined, but no significant effects on serum total, LDL and HDL cholesterol were found.

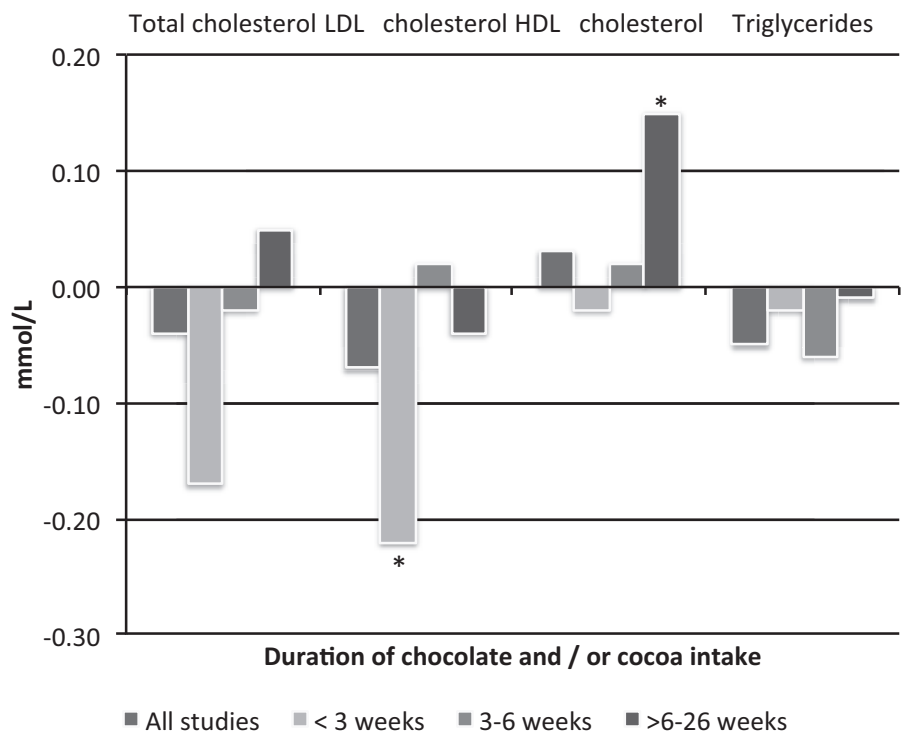

Figure 2.4. Effect of duration of chocolate and / or cocoa intake on serum total cholesterol, LDL cholesterol, $\mathrm{HDL}$ cholesterol, and triglyceride concentrations. Statistically significant differences between the groups with different duration are indicated with an asterisk. (from Hooper, L., Kay, C., Abdelhamid A., Kroon, P.A., Cohn, J.S., Rimm, E.B., and Cassidy A. Am J Clin Nutr., 95, 740-751, 2012).

Human studies have also shown an increased in vitro resistance of LDL to oxidation after intake of polyphenol-rich fractions from cocoa $(54,55)$. Furthermore, polyphenol-rich cocoa fractions increased fecal cholesterol excretion in rats (56).

\section{Trans-resveratrol}

The most widely known stilbene, resveratrol, is thought to exert several cardioprotective effects, among others the modulation of lipid and lipoprotein metabolism. Indeed, many in vitro or animal studies suggest that resveratrol has positive effects on proteins that are involved in reverse cholesterol transport. These proteins include PPARY, LXR $\alpha, 27-$ hydroxylase and ABCA1 (57), which are involved in cholesterol efflux. In hamsters, 
resveratrol may in vitro protect LDL against oxidation, downregulate HMG-CoA reductase, and increase the apolipoprotein $A-I$ to apoB ratio (58). Furthermore, resveratrol might enhance cholesterol efflux by preventing HDL particles from oxidation (Figure 2.5) (59). The possible anti-atherosclerotic effect of resveratrol might also be related to lower a reduced transfer of cholesteryl esters from HDL to VLDL and LDL through an inhibitory effect on CETP activity (60).

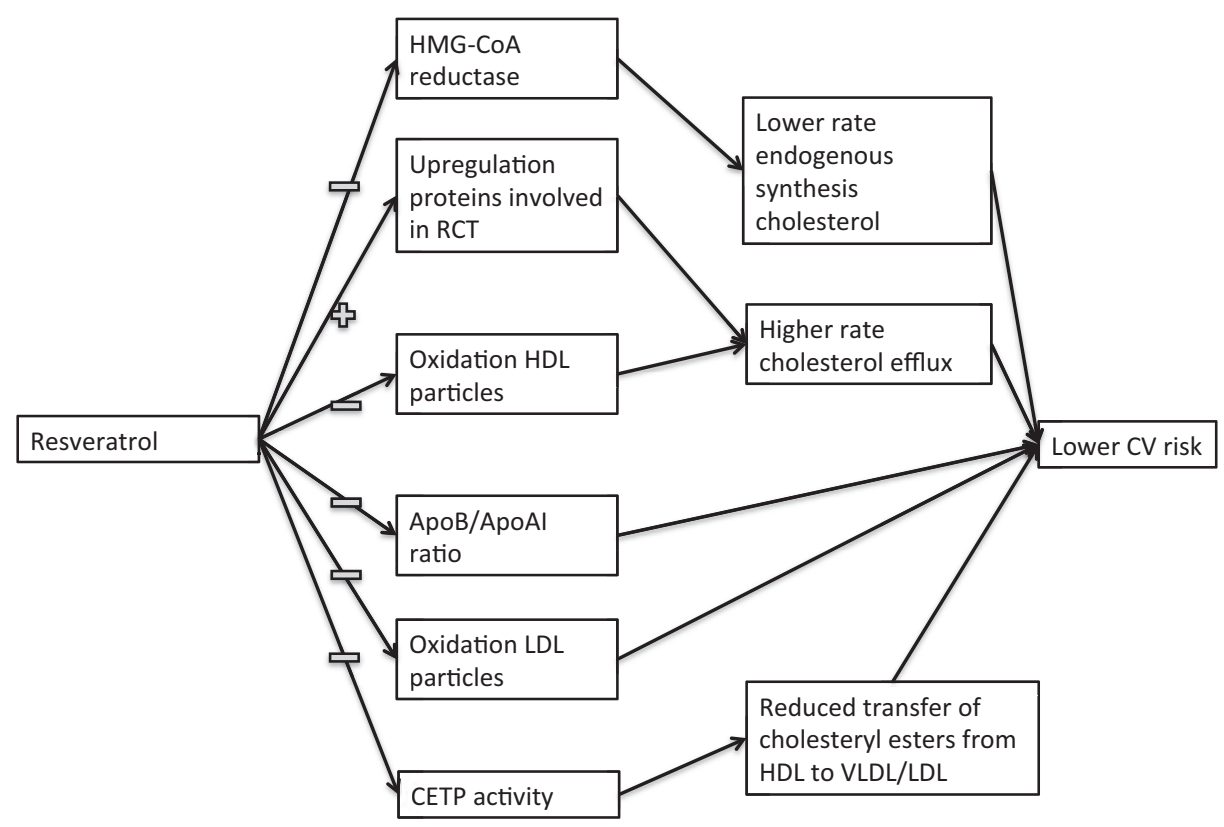

Figure 2.5. Proposed effects of resveratrol on cholesterol and apolipoprotein metabolism and cardiovascular risk as seen in animals. +; stimulating effect, -; inhibiting effect, RCT; reverse cholesterol transport, HDL; highdensity lipoprotein; ApoB; apolipoprotein B100; ApoAl; apolipoprotein AI, LDL; low-density lipoprotein, CETP; cholesteryl ester transfer protein, VLDL; very-low-density lipoprotein, CV; cardiovascular.

In humans, a recent meta-analysis using seven studies showed no statistically significant effect of the intake of purified trans-resveratrol and extracts containing resveratrol on serum total cholesterol, LDL cholesterol HDL cholesterol, and triglyceride concentrations (61). Results were not related to the dose of resveratrol used, study duration, or cardiovascular risk profile of the participants. However, the number of studies may have been too few to examine these potential sources of heterogeneity into detail. Also, none of the studies was specifically designed to examine the effects of resveratrol on the serum lipoprotein profile. Finally, in some of the studies subjects were on statin therapy, which may have masked any potential effects of resveratrol. Thus, before more 
definitive conclusions can be drawn, more powerful trials on the effects of transresveratrol supplementation on lipid metabolism in hyperlipidemic subjects are needed.

\section{Catechins and isoflavones}

Animal studies have shown positive effects on lipid metabolism of green tea catechins, of which epigallocatechin is the most abundantly present. These effects include reducing intestinal lipid absorption, promoting fecal cholesterol excretion, and inhibiting enzymes involved in hepatic cholesterol synthesis.

In humans, a significant reduction in total and LDL cholesterol after green tea catechin intake was found in a meta-analysis including 1415 subjects in twenty trials. This metaanalysis included studies with green tea catechin doses ranging between 145 and 3000 mg, while study duration varied between 3 and 24 weeks. Green tea catechins significantly lowered total cholesterol $(-0.14 \mathrm{mmol} / \mathrm{L} ; 95 \% \mathrm{Cl}:-0.25,-0.03 \mathrm{mmol} / \mathrm{L})$, and LDL cholesterol $(-0.14 \mathrm{mmol} / \mathrm{L} ; 95 \% \mathrm{Cl}:-0.26,-0.02 \mathrm{mmol} / \mathrm{L})$. No significant effects on HDL cholesterol and triglycerides were observed (62) (Figure 2.6).

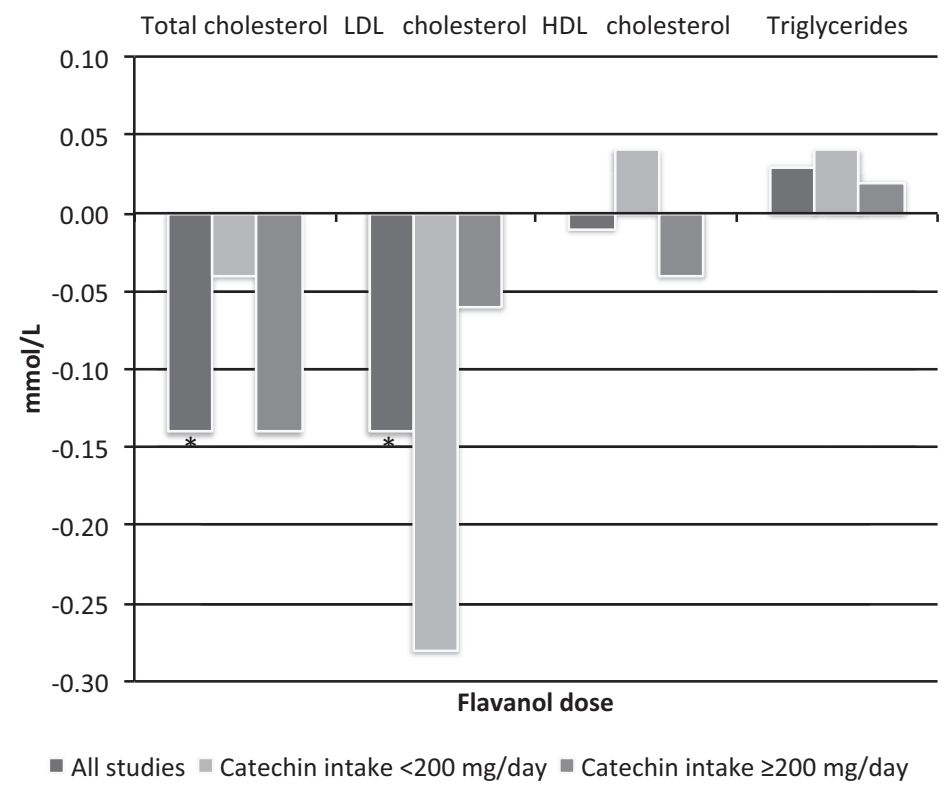

Figure 2.6. Effects of catechin intake on serum total cholesterol, LDL cholesterol, HDL cholesterol and triglyceride concentrations. A statistically significant $\left({ }^{*}\right)$ reduction in serum total and LDL cholesterol was found in all studies, while HDL and triglyceride concentrations did not change. Differences in effects between the two levels of intakes did not reach statistical significance. (from Kim, A., Chiu, A., Barone, M.K., Avino, D., Wang, F., Coleman, C.I., Phung, O.J. J. Am. Diet. Assoc., 111, 1720-1729, 2011). 
Regarding the effects of isolated soy isoflavones (in which the soy protein is absent) on lipid metabolism, a meta-analysis with 10 studies and 959 subjects did not suggest that these components do affect serum lipoprotein concentrations (63). However, soy protein containing intact isoflavones showed a beneficial effect on the lipid profile in two meta-analyses $(64,65)$.

\section{Effects of polyphenols on endothelial function}

The main focus of this paragraph will be on the polyphenol epicatechin, because this flavonol from cocoa has been studied most widely with respect to endothelial function. Furthermore, many recent studies have focused on the stilbene trans-resveratrol. Therefore, effects of this compound on endothelial function will be discussed as well.

\section{Flavonoids}

Regarding the positive effects of flavanols on endothelial function, a recent metaanalysis found a beneficial effect of chocolate, cocoa, and/or cocoa flavanols on flow mediated dilatation (FMD) (53). An acute effect - 2 hours after intake of chocolate or cocoa - of on average 3.19\% (95\% Cl: 2.04\%, 4.33\%) on FMD was reported. Epicatechin dose might play a role in this beneficial effect, as an increased epicatechin dose $(>100$ $\mathrm{mg} / \mathrm{d}$ ) from the experimental products showed a more pronounced effect on acute FMD compared to lower epicatechin doses. A mean difference in FMD of $1.34 \%(95 \% \mathrm{Cl}$ : $1.00 \%, 1.68 \%$ ) was observed after chronic intake (3-26 wks) of chocolate/cocoa.

Another meta-analysis also reported beneficial effects of the intake of flavonoid-rich cocoa on several cardiovascular risk factors. In this study, an increase of $1.53 \%$ (95\% Cl: $0.67 \%, 2.40 \%$ ) in FMD after chronic (2-18 wks) consumption of flavonoid-rich cocoa was found (66). One meta-analysis examined the effect of flavonoid-subclasses on FMD. The acute mean effect of epicatechin on FMD was 3.22\% (95\% Cl: 1.94\%, 4.50\%), whereas for total flavonoids (the sum of flavanols, catechol flavonoids, procyanidins, epicatechin and catechins) an acute effect of $2.33 \%(95 \% \mathrm{Cl}: 1.58 \%, 3.08 \%)$ was reported. Also, in studies that reported the longer-term effects ( $\geq 2$ weeks intervention), epicatechin intake from the experimental products showed a somehow larger improvement $(0.94 \%$; $95 \% \mathrm{Cl}: 0.47 \%, 1.42 \%)$ on FMD than total flavonoid intake $(0.73 \% ; 95 \% \mathrm{Cl}: 0.17 \%, 1.30 \%)$ (Figure 2.7) (67). 


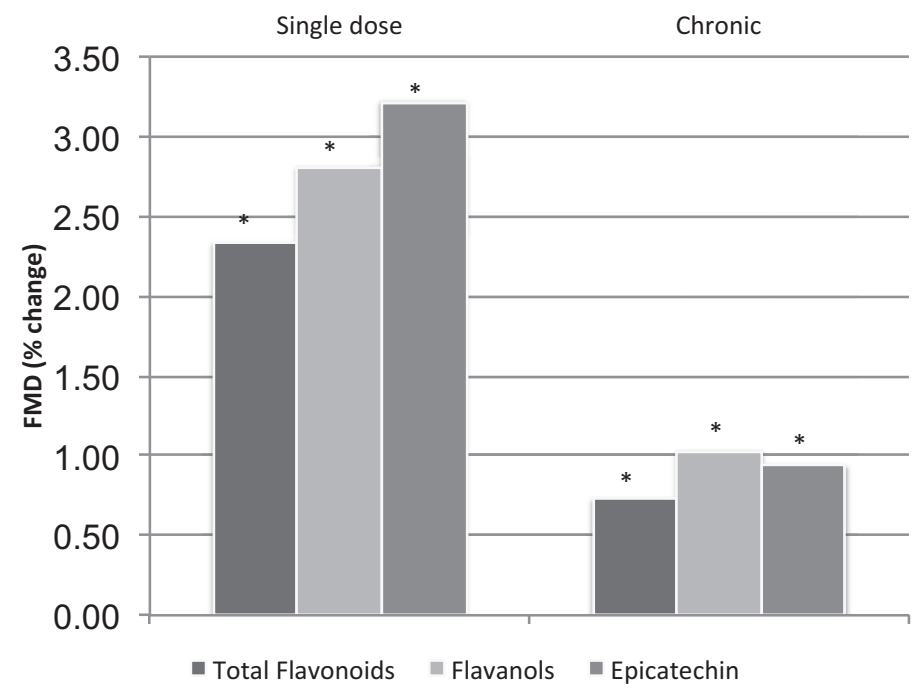

Figure 2.7. Effect of single dose and chronic (minimum of 2 weeks intervention) flavonoid intake on \% change in FMD. All results were statistically significant $\left({ }^{*}\right)$. FMD; flow-mediated dilation (from Kay, C.D., Hooper, L., Kroon, P.A., Rimm, E.B., Cassidy, A. Mol. Nutr. Food Res., 00, 1-12, 2012).

The mechanisms to explain the beneficial effect of cocoa polyphenols in general or of epicatechin in particular on FMD have not been elucidated yet. It has been suggested that epicatechin enhances NO bioavailability and therefore exerts a positive effect on FMD. This increase in NO might be the result of an increased expression and/or activity of eNOS, but also of changes in NO bioavailability or changes in the expression of eNOSrelated proteins. This eNOS activation is likely to be mediated via the calcium/calmodulin pathway, as described in Figure 2.8 (68). 


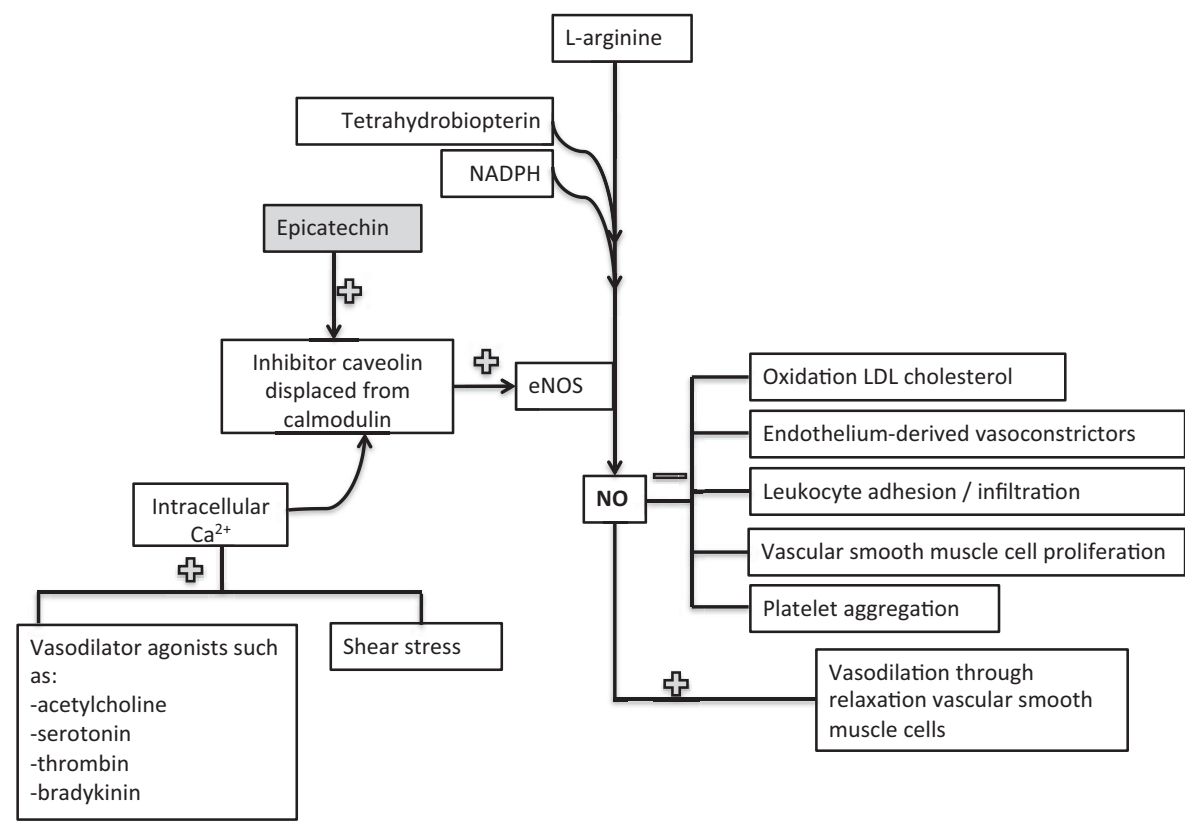

Figure 2.8. Effects of NO on endothelial function and the possible role of epicatechin in this process. eNOS; endothelial nitric oxide synthase, NO; nitrogen oxide, NADPH; Nicotinamide adenine dinucleotide phosphate, LDL; low-density lipoprotein (based on Ramirez-Sanchez, I., Maya, L., Ceballos, G., Kroon, P.A., Vilarreal, F. Hypertension, 55, 1398-1405, 2010).

Other suggested pathways that positively influence endothelial function include the antioxidant capacity of flavonoids, which leads to lower oxidative stress levels in vivo and a reduced endothelial adhesion molecule expression in vitro (69). Furthermore, cocoa and chocolate showed positive effects on fasting insulin and $\operatorname{HOMA-IR}(53,70)$. This finding might be related to the positive effect of chocolate and cocoa on endothelial function, as these processes share common pathways (70).

The upregulation of NO production has several positive effects on the vasculature. First, NO exerts antihypertensive effects through vasodilation. Secondly, the antithrombotic effect of NO is reflected by the inhibition of platelet aggregation. This effect might be mediated by the inhibition of cyclooxygenase-2 (COX-2), which catalyzes the synthesis of prostaglandin $E_{2}\left(P G E_{2}\right)$. Decreasing $P E_{2}$ synthesis has a beneficial effect on platelet aggregation. Finally, the ability of NO to prevent leukocyte adhesion to the endothelium, the reduction of LDL oxidation and the inhibition of vascular smooth muscle cell proliferation may contribute to the anti-atherosclerotic effects of NO production. 


\section{Trans-resveratrol}

A recently published article showed an acute, dose-dependent, FMD-improving effect of resveratrol (71). Here, resveratrol was given to 19 overweight or obese men or postmenopausal women who were borderline hypertensive (systolic blood pressure between 130-160 mmHg or diastolic pressure between $85-100 \mathrm{mmHg}$ ), but did not receive any treatment. Subjects received six capsules containing in total 30, 90 or $270 \mathrm{mg}$ transresveratrol. The study had a double-blind, randomized cross-over design and subjects were asked to consume the indicated doses and a placebo at weekly intervals.

Several in vitro and animal studies have shown that resveratrol may stimulate eNOS and improve NO bioactivity (72). Except for the effects of resveratrol on NO production, the compound is also thought to exert positive effects on endothelial dysfunction by inhibiting NFKB, leading to lower cytokine production and less vascular inflammation (72). Furthermore, platelet aggregation might be prevented by resveratrol through prevention of eNOS acetylation and sirtuin type 1-activation.

\section{Polyphenol metabolism}

\section{Flavonoids}

Flavonoids, except for catechins, are present in the diet as $\beta$-glycosides. Flavonoid glycosides, but not glucosides, are thought to pass the small intestine, followed by hydrolysis to aglycones by enterobacteria in the cecum and colon (73). Absorption of flavonoid aglycones in the large intestine is facilitated through their lipophilicity by passage across the phospholipid bilayer of the cellular membranes. After entering the circulation, the flavonoid aglycones are further metabolized in the liver (O-methylation, glucuronidation, and/or sulfation). Part of the metabolites will be excreted in the bile, followed by a return to the intestinal lumen, where they might either be reabsorbed by intestinal cells or excreted into faeces. Glucosides are thought to be absorbed from the small intestine, which leads to higher plasma values because of the higher absorption efficiency. Catechins are present in foods as aglycones or esterified with gallic acid. Both forms are absorbed from the small intestine.

Bioavailability of flavonol glycosides differs among the separate classes. Time to reach plasma peak concentrations vary between less than $0.5 \mathrm{~h}$ and $9 \mathrm{~h}$, with the highest bioavailability of quercetin glucosides from onions (74). Flavonols that are particularly present in cocoa, (epi)-catechin and procyanidin, reach a peak concentration after 2 hours (75). 


\section{Trans-resveratrol}

After oral administration, trans-resveratrol is mainly absorbed in the duodenum and, to a lesser extent, the jejunum. After that, metabolic conversion via intestinal and hepatic conjugation starts. Both intestinal subcellular fractions and liver cells are capable of glucuronidation and sulfation of resveratrol, although glucuronidation prevails over sulfation in the liver. This results in low plasma levels of free resveratrol, whereas the major metabolites (trans-)resveratrol-3-O-sulfate, (trans-)resveratrol-3-O-glucuronide and (trans-)resveratrol-4'-O-glucuronide are mainly found in plasma after resveratrol intake.

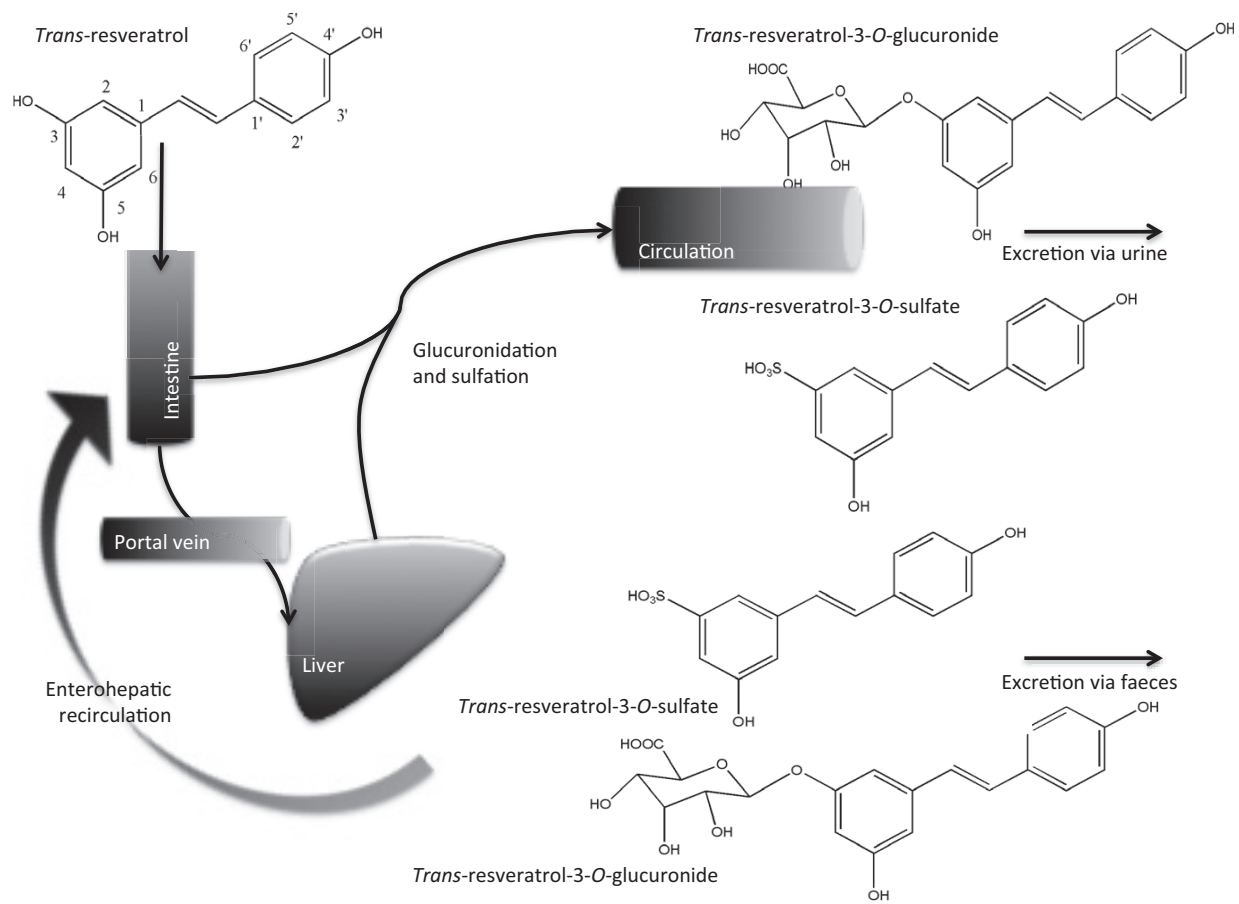

Figure 2.9. Trans-resveratrol is absorbed in the intestine, followed by glucuronidation and sulfation by liver and intestinal subcellular fractions, which results (amongs others) in the formation of trans-resveratrol-3-0glucuronide and trans-resveratrol-3-O-sulfate. These conjugates can be excreted via urine or undergo enterohepatic recirculation. During this process, trans-resveratrol conjugates are returned to the liver via the bile duct, intestines and portal vein and can either be excreted via faeces or re-enter the circulation and excreted via urine. 
A second resveratrol and resveratrol-metabolite peak is observed 6 hours after resveratrol intake. This is explained by enterohepatic recirculation of conjugated resveratrol metabolites. During this process, resveratrol is metabolized in the liver and its conjugates are excreted in the bile. This is followed by reabsorption of the conjugates in the small intestine and subsequent return to the liver or excretion via faeces (Figure 2.9).

Resveratrol absorption is at least 70\%, and the compound and its metabolites are mainly excreted via urine. The absorption is delayed when resveratrol is taken with foods, especially with a high-fat meal.

\section{Dosing regimen and adverse effects}

A recent scientific opinion by the European Food Safety Authority (EFSA) stated that 200 mg of cocoa flavanols should be consumed daily in order to "maintain endotheliumdependent vasodilation, which contributes to normal blood flow" (76). This amount of flavanols can be consumed through $10 \mathrm{~g}$ of high-flavanol-dark chocolate. For the other components discussed in this chapter, no effective doses have been formulated by health authorities.

\section{Conclusions}

Scientific interest in the relation between polyphenol intake and cardiovascular health has considerably grown during the past decades. Polyphenols can be found in numerous products of which flavonoids from cocoa, the stilbene trans-resveratrol from black grapes, green tea catechins, and soy isoflavones have been most widely studied.

Green tea catechins were shown to improve the serum lipid profile by decreasing total cholesterol and LDL cholesterol. Isolated soy isoflavones did not affect serum lipoprotein concentrations, whereas soy protein containing intact isoflavones may have a beneficial effect on the lipid profile. Consumption of cocoa products or dark chocolate for two weeks showed a significant decrease in LDL cholesterol. This effect was not found after 4-12 weeks consumption of these products, which raises questions on the clinical usefulness of this finding. HDL cholesterol showed an increase in longer-term studies. Next to this, beneficial effects of flavonoids from cocoa on FMD were reported, which underlines the positive role of these compounds in cardiovascular risk reduction. Transresveratrol does not affect serum lipid and lipoprotein concentration, but may improve vascular health. However, more studies are needed to substantiate these findings. Several mechanisms underlying these effects of the various polyphenols have been proposed, but an unambiguous explanation cannot be given yet. 


\section{Chapter 3}

Resveratrol does not influence metabolic risk markers related to cardiovascular health in overweight and slightly obese subjects: a randomized, placebo-controlled crossover

trial 


\section{Abstract}

Background In vitro and animal studies have shown positive effects of resveratrol on lipid and lipoprotein metabolism, but human studies specifically designed to examine these effects are lacking.

Objective The primary outcome parameter of this study in overweight and slightly obese subjects was the effect of resveratrol on apoA-I concentrations. Secondary outcome parameters were effects on other markers of lipid and lipoprotein metabolism, glucose metabolism, and markers for inflammation and endothelial function.

Design This randomized, placebo-controlled crossover study was conducted in 45 overweight and slightly obese men $(n=25)$ and women $(n=20)$ with a mean age of $61 \pm$ 7 years. Subjects received in random order resveratrol (150 mg per day) or placebo capsules for 4 weeks, separated by a 4-week wash-out period. Fasting blood samples were collected at baseline and at the end of each intervention period.

Results Compliance was excellent as indicated by capsule count and changes in resveratrol and dihydroresveratrol concentrations. No difference between resveratrol and placebo was found in any of the fasting serum or plasma metabolic risk markers (mean \pm SD for differences between day 28 values of resveratrol vs. placebo: apoA-l; $0.00 \pm 0.12 \mathrm{~g} / \mathrm{L}(\mathrm{P}=0.791)$, apoB100; $-0.01 \pm 0.11 \mathrm{~g} / \mathrm{L}(\mathrm{P}=0.545), \mathrm{HDL}$ cholesterol; $0.00 \pm$ $0.09 \mathrm{mmol} / \mathrm{L}(P=0.721), \mathrm{LDL}$ cholesterol $-0.03 \pm 0.57 \mathrm{mmol} / \mathrm{L}(P=0.718)$, triacylglycerol; $0.10 \pm 0.54 \mathrm{mmol} / \mathrm{L}(P=0.687)$, glucose; $-0.08 \pm 0.28 \mathrm{mmol} / \mathrm{L}(P=0.064)$, insulin; $-0.3 \pm 2.5$ $\mathrm{mU} / \mathrm{L}(\mathrm{P}=0.516))$. Also, no effects on plasma markers for inflammation and endothelial function were observed. No adverse events related to resveratrol intake were observed. Conclusion $150 \mathrm{mg}$ of daily resveratrol intake for 4 weeks does not change metabolic risk markers related to cardiovascular health in overweight and slightly obese men and women. Effects on glucose metabolism warrant further study. 


\section{Introduction}

The many approaches that exist to lower cardiovascular risk are mainly aimed at lowering LDL cholesterol concentrations. Based on epidemiological studies $(32-34,77)$, increasing HDL cholesterol concentrations may also reduce cardiovascular risk, but this concept has been challenged by intervention studies (78-80). However, a recent metaanalysis has suggested that increasing apolipoprotein $\mathrm{A}-\mathrm{I}$ (apoA-I) ${ }^{4}$ concentrations is a more important target to reduce the risk of major cardiovascular events than increasing $\mathrm{HDL}$ cholesterol concentrations (35). ApoA-I is the major protein constituent of the HDL particle, which plays a crucial role in many of the favorable effects attributed to HDL particles, including - next to their fundamental role in reverse cholesterol transport anti-inflammatory, anti-thrombotic and anti-oxidative effects $(36,37)$.

Epidemiological studies have shown a positive association between higher polyphenol intakes and reduced cardiovascular risk $(46,81)$. This association may be due to increased intakes of trans-resveratrol (3,5,4'-trihydroxystilbene), a polyphenol found in the skin of black grapes. Resveratrol may influence numerous mechanisms, which act on the progression as well as on the regression of atherosclerosis (82). In fact, in vitro and animal studies have shown many positive effects of resveratrol on genes and proteins involved in lipid and glucose metabolism, including increases in the activities of peroxisome proliferator-activated receptor $\alpha$ (PPAR $\alpha)$ and PPARY $(83,84)$.

While the results from in vitro and animal studies are promising, and an HDL cholesterol elevating effect of berries (85) and red wine (86), both of which contain resveratrol, has been reported, human studies with the primary aim to examine the effects of resveratrol on lipid and lipoprotein metabolism are scarce. Furthermore, human data onto the effect of resveratrol on markers for metabolic risk is limited and results are conflicting (87-89). Therefore, the primary aim of the present study was to evaluate the effect of $4 \mathrm{wk}$ resveratrol supplementation $(150 \mathrm{mg} / \mathrm{day}$, 99\% pure transresveratrol [resVida provided by DSM Nutritional Products Ltd. (Kaiseraugst, Switzerland]) on apoA-I concentrations in overweight and slightly obese subjects with low HDL cholesterol concentrations. Secondary aims were to assess effects on other markers of lipid and lipoprotein metabolism, glucose metabolism, and markers for inflammation and endothelial function. 


\section{Subjects and methods}

\section{Subjects}

Subjects were recruited via posters in university and hospital buildings and by advertisements in local newspapers. In addition, men and women who had participated in earlier studies at the Department of Human Biology (Maastricht University) were approached. Subjects came to university for two screening visits with an interval of $\geq 3$ days. Fasting blood was sampled on both occasions for lipoprotein and glucose analyses. Also, height, weight and blood pressure were determined. Furthermore, subjects were asked to complete a general and medical questionnaire. Inclusion criteria were as follows: age between 45 and 70 y; mean high-density lipoprotein cholesterol concentration $<1.21 \mathrm{mmol} / \mathrm{L}$ for men and $<1.53 \mathrm{mmol} / \mathrm{L}$ for women, in order to include subjects with HDL cholesterol concentrations below mean values found in the PROCAM study (32); mean serum total cholesterol concentration < $8.0 \mathrm{mmol} / \mathrm{L}$; plasma glucose < $7.0 \mathrm{mmol} / \mathrm{L}$; body mass index between $25-35 \mathrm{~kg} / \mathrm{m}^{2}$; non-smoker; willing to abstain from resveratrol-rich products two weeks prior to the study and during the study; stable body weight (weight gain or loss $<3 \mathrm{~kg}$ in three months prior to screening visit); no indication for treatment with cholesterol-lowering drugs according to Dutch Cholesterol Consensus; no use of medication or a prescribed diet known to affect serum lipid or glucose metabolism; no active cardiovascular disease or recent ( $<6$ months) event such as acute myocardial infarction or cerebrovascular accident; willing to stop consumption of vitamin supplements, fish oil capsules or products rich in plant stanol or sterol esters two weeks prior to and during the study; self-reported high alcohol consumption (> 21 units / week for men or > 14 units / week for women); no abuse of drugs; no pregnant or breastfeeding women; no participation in another biomedical study within 1 month prior to the first screening visit; not having donated blood (as blood donor) within 1 month prior to the first screening visit, planning to donate blood during the study or within one month after finishing the study. Finally, subjects who were impossible or difficult to puncture as evidenced during the screening visits could not participate. Participant were informed about the aim of the study and nature and risk of the experimental procedures before their written informed consent was obtained. This study was conducted according to the guidelines laid down in the Declaration of Helsinki, approved by the Ethics Committee of the Maastricht University Medical Centre, and registered on 17 January 2011 at ClinicalTrials.gov as NCT01364961. 


\section{Study design and products}

The study had a double-blind, randomized, placebo-controlled, cross-over design with two 4-wk intervention periods separated by a 4-wk wash-out period. Subjects were allocated to start with either resveratrol or placebo capsules according to a preestablished computer generated randomization scheme, and were asked to take two capsules daily during lunch and dinner. Resveratrol (150 mg/day; resVida ${ }^{\mathrm{TM}}$, 99.9\% transresveratrol) and placebo capsules were kindly provided by DSM Nutritional Products Ltd. (Kaiseraugst, Switzerland). Capsules were provided in weekly containers, coded with either ' $A$ ' or ' $B$ ' to blind subjects and investigators. Four containers were provided to the volunteers at the start of each intervention periods and subjects were asked to return all containers including any unused capsules, which were counted as a measure of compliance. Two weeks prior to the study and throughout the total study period, subjects were asked to abstain from resveratrol-containing products (such as grapes, wine, berries, peanuts, peanut butter, soy and soy products and pomegranate). At the end of both experimental periods, subjects completed a food-frequency questionnaire (FFQ) in which food intake from the previous 4 wk was recorded. A dietitian immediately checked the FFQs and calculated energy and nutrient intakes of the subjects using the Dutch food-composition tables. Subjects were asked to record any signs of illnesses, use of medication or alcohol consumption in a study diary.

\section{Blood sampling}

Fasting blood samples were taken by venipuncture at the start and at day 25 of each intervention period. At day 28 blood was sampled through an intravenous catheter using a Vacutainer system (Becton, Dickinson and Company, Franklin Lanes, NY, USA). On days preceding blood drawings, subjects were asked to avoid the intake of drinks containing alcohol or caffeine, and not to take part in any strenuous activity. Blood samples were taken from a forearm vein after an overnight fast (no food or drink after 8 PM, except for water), by the same person, and at the same location. Serum separator tubes (Becton, Dickinson and Company, Franklin Lanes, NY, USA) were used for the analysis of lipids and lipoproteins, insulin and hsCRP. Serum tubes were kept at room temperature for at least 30 minutes after sampling to allow clotting. Serum was obtained by centrifugation at $1300 \times \mathrm{g}$ for 15 minutes at $21^{\circ} \mathrm{C}$. EDTA-coated tubes (Becton, Dickinson and Company, Franklin Lanes, NY, USA) were used for measurement of inflammatory markers, resveratrol and dihydroresveratrol, and clinical chemistry measurements. Sodium fluoride (NaF) tubes (Becton, Dickinson and Company, Franklin Lanes, NY, USA) were used for analysis of plasma glucose. After blood drawing, NaF and EDTA-coated tubes were kept on ice and centrifuged within 30 minutes. Plasma was obtained by centrifugation at $1300 \times \mathrm{g}$ for 15 minutes at $4^{\circ} \mathrm{C}$. Serum and plasma samples were portioned into aliquots, snap-frozen in liquid $\mathrm{N}_{2}$, and stored at $-80^{\circ} \mathrm{C}$. Hemostatic and 
hematologic measurements were performed in whole blood from citrate-filled and EDTA-coated tubes respectively (Becton, Dickinson and Company, Franklin Lanes, NY, USA). These measurements were performed within 4 hours after blood drawing.

\section{Clinical and laboratory measurements}

All samples were analyzed for serum apoA-I (Horiba ABX, Montpellier, France), apolipoprotein B-100 (apoB-100; Horiba ABX, Montpellier, France), high-sensitive Creactive protein (hSCRP; Horiba ABX, Montpellier, France), total cholesterol (CHOD-PAP method; Roche Diagnostics, Mannheim, Germany), HDL cholesterol (precipitation method; Roche Diagnostics, Mannheim, Germany) and triacylglycerol with correction for free glycerol (GPO Trinder; Sigma-Aldrich Corp., St. Louis, MO, USA). LDL cholesterol was calculated by using the Friedewald formula (90). Furthermore, fasting EDTA-plasma samples were analyzed for insulin concentrations (RIA; Millipore, Billerica, MA, USA) and fasting Na-F plasma samples were analyzed for glucose concentrations (Horiba ABX, Montpellier, France). IL-6, TNF $\alpha$, E-selectin, thrombomodulin, P-selectin, ICAM-3, sICAM1 and sVCAM-1 were measured by commercially available Multi Spot ELISA kits (Meso Scale Discovery, Rockville, MD, USA).

Liver and kidney function, and general health parameters (ureum, creatinin, alkaline phosphatase (ALP), $\gamma$-glutamyl transpeptidase (GGT), aspartate aminotransferase (ASAT), alanine aminotransferase (ALAT), total bilirubin, total protein, albumin, sodium, potassium, calcium, phosphorus and chloride) were measured in serum on a Beckman Coulter Synchron LX20 PRO Clinical System (Beckman Coulter Inc., Fullerton, CA, USA). Hematologic parameters (erythrocyte-, thrombocyte-, and leucocyte count, haemoglobin, haematocrit, erythrocyte mean corpuscular volume, erythrocyte mean corpuscular haemoglobin (concentration), and erythrocyte relative distribution weight) were measured on a Beckman Coulter LH750 (Beckman Coulter Inc., Fullerton, CA, USA) until October 2011. From October 2011 onwards, measurements were performed on a Sysmex XE-5000 System (Sysmex Corporation, Kobe, Japan). Haemostatic measurements (prothrombin time (PT) and activated partial thromboplastin time (aPTT)) were performed on a Sysmex CA-7000 Analyzer (Sysmex Corporation, Kobe, Japan).

Body weight was measured at each visit in the morning, after an overnight fast. Seated blood pressure was measured each visit (Omron M7, Omron Healthcare Co., Ltd, Kyoto, Japan), after 5 minutes rest. Measurements were performed four times and the average of the last three measurements is reported.

The degree of insulin resistance was estimated by HOMA $\mathrm{IR}_{\mathrm{R}}$ as described (91).

Compliance was checked by capsule count and by measuring total resveratrol and dihydroresveratrol concentrations (both free and conjugated forms) in plasma by liquid 
chromatography-mass spectrometry at day 28 of both intervention periods by DSM Nutritional Products, Ltd. Kaiseraugst, Switzerland.

\section{Statistical analyses}

Before the start of the study, it was calculated that the statistical power to detect a true difference of at least $0.05 \mathrm{~g} / \mathrm{L}$ in serum apo A-I concentrations between the experimental and control period was over $80 \%$, when 45 subjects were included at $P=0.05$. For the calculations, a within-subject variability of $0.12 \mathrm{~g} / \mathrm{L}$ in serum apo A-I concentrations was used. As the expected drop-out rate was 10\%, a total of 50 men and women were included.

A Shapiro-Wilk normality test was performed to examine if the changes between days 28 of the resveratrol and control periods were normally distributed. A student's paired t-test was used to compare the values of the experimental and control periods from day 28 in normally distributed data. When data were not normally distributed, a Wilcoxon signed-rank test was used. Results, presented as means \pm standard deviations (SD), were considered to be statistically significant if $p<0.05$. Statistical analyses were performed using SPSS 19.0 for Mac Os X (SPSS Inc., Chicago, IL, USA).

Table 3.1 Baseline characteristics of participants ${ }^{1,2}$

\begin{tabular}{|c|c|c|c|c|c|c|c|c|c|}
\hline \multirow[b]{2}{*}{ Age (y) } & \multicolumn{3}{|c|}{$\begin{array}{c}\text { All } \\
(N=45)\end{array}$} & \multicolumn{3}{|c|}{$\begin{array}{l}\text { Males } \\
(\mathrm{N}=25)\end{array}$} & \multicolumn{3}{|c|}{$\begin{array}{l}\text { Females } \\
(\mathrm{N}=20)\end{array}$} \\
\hline & 60 & \pm & 7 & 62 & \pm & 7 & 59 & \pm & 7 \\
\hline $\mathrm{BMI}(\mathrm{kg} / \mathrm{m} 2)$ & 28.8 & \pm & 3.2 & 28.5 & \pm & 2.9 & 29.2 & \pm & 3.6 \\
\hline Systolic BP (mmHg) & 136 & \pm & 17 & 138 & \pm & 16 & 135 & \pm & 18 \\
\hline Diastolic BP (mmHg) & 88 & \pm & 9 & 88 & \pm & 10 & 88 & \pm & 9 \\
\hline Heart rate (BPM) & 67 & \pm & 8 & 67 & \pm & 8 & 68 & \pm & 7 \\
\hline Glucose (mmol/L) & 5.66 & \pm & 0.53 & 5.71 & \pm & 0.57 & 5.59 & \pm & 0.48 \\
\hline Total cholesterol (mmol/L) & 6.38 & \pm & 1.00 & 6.20 & \pm & 1.01 & 6.60 & \pm & 0.97 \\
\hline HDL cholesterol (mmol/L) & 1.14 & \pm & 0.23 & 1.00 & \pm & 0.17 & 1.30 & \pm & 0.18 \\
\hline
\end{tabular}

${ }^{2} \mathrm{BP}$, blood pressure; BPM: beats per minute

\section{Results}

\section{Subject characteristics and compliance}

A flow diagram of participants throughout the study is shown in Figure 3.1. After screening, 50 subjects were eligible for participation and started the study. Two female subjects discontinued intervention because of personal reasons, 1 male subject dropped out because of a headache, one female subject dropped out because of stomach complaints and another female subject started statin treatment after randomization. A 
total of five subjects dropped out either during or straight after the first intervention period. Three of these subjects dropped out during the control period and two subjects during the resveratrol period. A total of 45 subjects ( 25 men and 20 women) completed the study. Baseline characteristics of study participants are shown in Table 3.1. Fourteen subjects used medication such as antidepressants, antihypertensives or antacids throughout the study without a change in dosage.

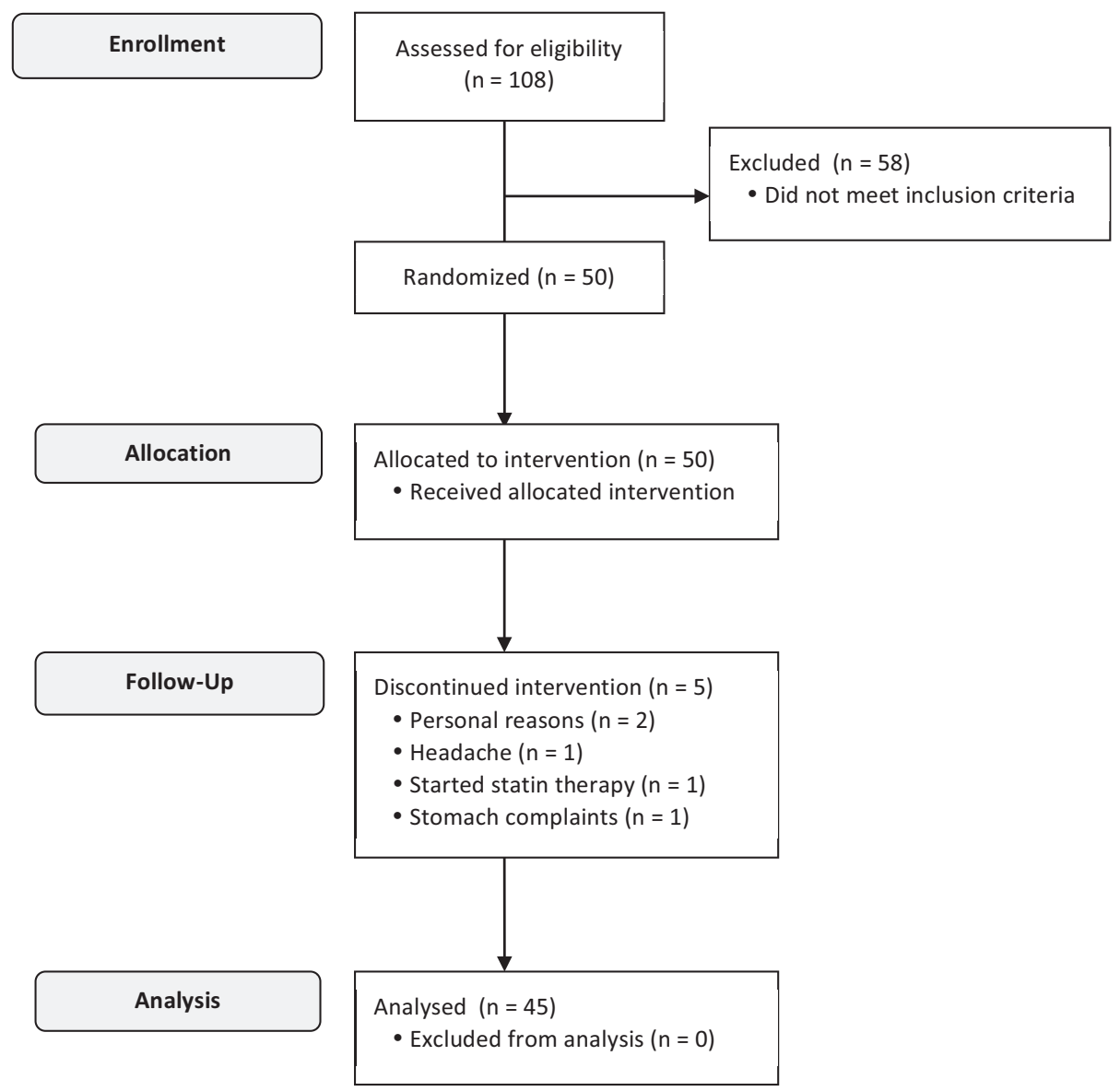

Figure 3.1. Subject flow chart.

As evidenced from pill count, compliance ranged between $88 \%$ and $111 \%$ and was on average 99\% during both the control and resveratrol period. To further check for compliance, free and total (sum of conjugated and free resveratrol) resveratrol and dihydroresveratrol (DHR) concentrations were measured in all plasma samples. Free 
resveratrol and free DHR were not detectable, except in all plasma samples from one subject. Total resveratrol and DHR were detectable in three subjects during the control period and in one subject at the start of the resveratrol period. At day 28 of the resveratrol period, mean total resveratrol concentrations were $197 \pm 185 \mathrm{ng} / \mathrm{mL}$ and those of DHR $408 \pm 298 \mathrm{ng} / \mathrm{mL}$. During the entire experimental period, one subject had no measurable plasma concentrations of total resveratrol or total DHR. Two subjects had no measurable resveratrol and DHR concentrations at day 28 , while these compounds were present at day 25. Another two subjects had no measurable DHR concentrations during the total study period. For all subjects, plasma total resveratrol concentrations ranged between 0 and $1080 \mathrm{ng} / \mathrm{mL}$, while total DHR concentrations varied between 0 and $1070 \mathrm{ng} / \mathrm{mL}$ during the resveratrol period. Individual resveratrol and $\mathrm{DHR}$ concentrations are shown in Figure 3.2.

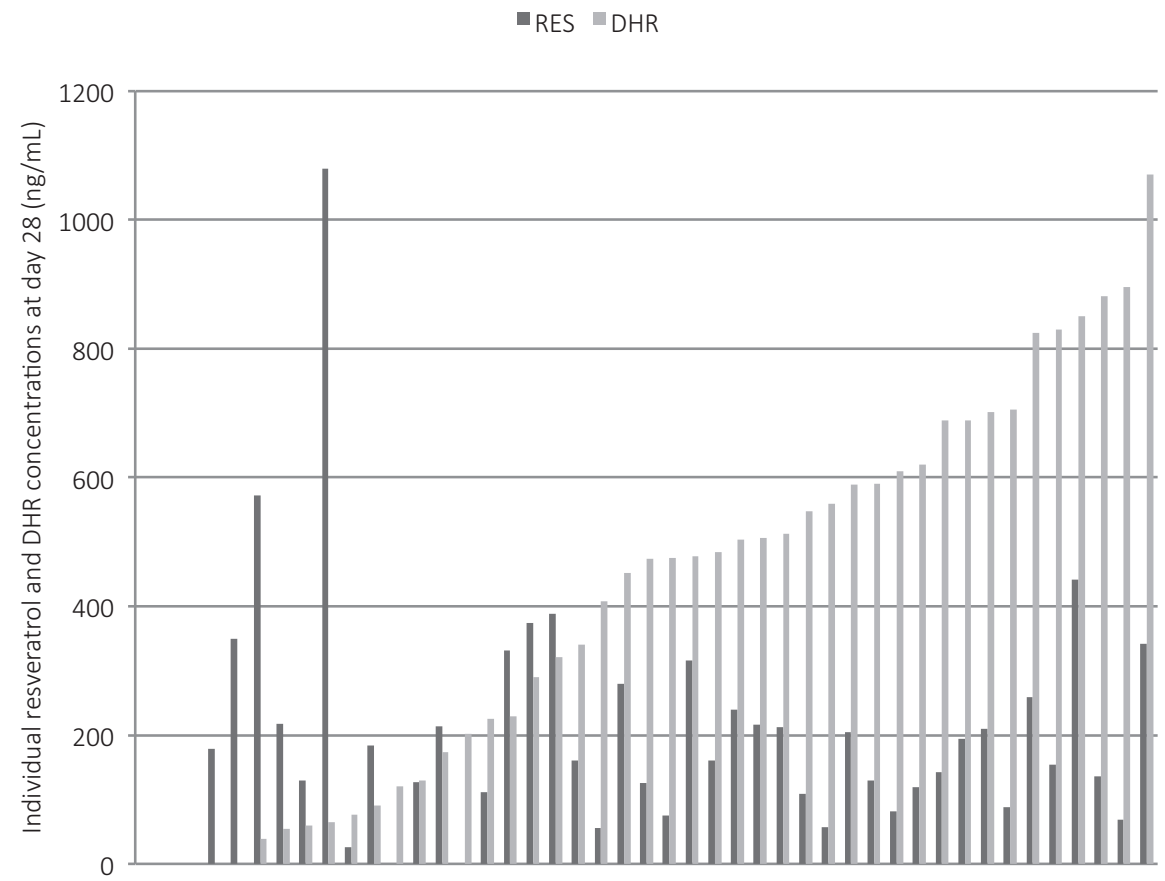

Figure 3.2. Individual total resveratrol (RES) and total dihydroresveratrol (DHR) concentrations at day 28. 
Table 3.2 Dietary intake during the placebo and resveratrol period, as estimated with foodfrequency questionnaires

\begin{tabular}{|c|c|c|c|c|c|c|c|c|c|c|}
\hline \multirow[b]{2}{*}{ Energy (MJ/d) } & \multicolumn{3}{|c|}{ Placebo } & \multicolumn{3}{|c|}{ Resveratrol } & \multicolumn{3}{|c|}{ Difference } & \multirow{2}{*}{$\begin{array}{l}P \text { value } \\
0.180\end{array}$} \\
\hline & 9.5 & \pm & 2.9 & 9.9 & \pm & 2.8 & 0.4 & \pm & 2.0 & \\
\hline Energy $(\mathrm{kcal} / \mathrm{d}) 2$ & 2273 & \pm & 694 & 2373 & \pm & 678 & 100 & \pm & 482 & 0.190 \\
\hline Carbohydrate (En\%) & 45.9 & \pm & 7.1 & 44.8 & \pm & 7.7 & -1.1 & \pm & 5.1 & 0.165 \\
\hline Protein (En\%) & 16.0 & \pm & 3.2 & 15.5 & \pm & 3.2 & -0.5 & \pm & 2.6 & 0.205 \\
\hline Total Fat (En\%) & 36.4 & \pm & 6.4 & 37.7 & \pm & 6.8 & 1.3 & \pm & 4.9 & 0.081 \\
\hline SFAs (En\%) & 12.0 & \pm & 2.7 & 12.2 & \pm & 2.6 & 0.2 & \pm & 2.6 & 0.608 \\
\hline MUFAs (En\%)2 & 12.0 & \pm & 2.6 & 12.9 & \pm & 3.4 & 0.9 & \pm & 2.3 & 0.022 \\
\hline PUFAs (En\%) & 9.1 & \pm & 3.0 & 9.3 & \pm & 3.5 & 0.2 & \pm & 2.9 & 0.725 \\
\hline Alcohol (En\%)2 & 1.9 & \pm & 3.1 & 2.2 & \pm & 3.7 & 0.3 & \pm & 1.6 & 0.478 \\
\hline Dietary fiber (g/d) & 24.9 & \pm & 7.7 & 24.6 & \pm & 6.1 & -0.3 & \pm & 5.2 & 0.716 \\
\hline Cholesterol (mg/d) & 201 & \pm & 71 & 202 & \pm & 68 & -1 & \pm & 60 & 0.884 \\
\hline
\end{tabular}

Values are means \pm SD

${ }^{1} \mathrm{~N}=45$.

${ }^{2}$ These parameters were tested by a Wilcoxon signed-rank test for non-normal distributed data.

Table 3.3 Effect of 4-wk resveratrol intake on serum lipids and lipoproteins ${ }^{1}$

\begin{tabular}{lllllllllllll}
\hline & \multicolumn{3}{c}{ Placebo } & \multicolumn{3}{c}{ Resveratrol } & \multicolumn{3}{c}{ Difference } & \multicolumn{2}{c}{ P value } \\
\hline Total cholesterol (mmol/L) & 5.70 & \pm & 0.90 & 5.69 & \pm & 0.83 & -0.02 & \pm & 0.62 & 0.870 \\
LDL cholesterol (mmol/L) & 3.83 & \pm & 0.84 & 3.77 & \pm & 0.75 & -0.06 & \pm & 0.59 & 0.530 \\
HDL cholesterol (mmol/L) & 1.15 & \pm & 0.23 & 1.15 & \pm & 0.24 & -0.01 & \pm & 0.09 & 0.696 \\
Total:HDL cholesterol ratio & 5.14 & \pm & 1.31 & 5.16 & \pm & 1.24 & 0.02 & \pm & 0.58 & 0.863 \\
Triacylglycerol (mmol/L) & 1.58 & \pm & 0.72 & 1.68 & \pm & 0.77 & 0.10 & \pm & 0.54 & 0.048 \\
Apolipoprotein A-I (g/L) & 1.28 & \pm & 0.19 & 1.27 & \pm & 0.20 & 0.00 & \pm & 0.12 & 0.670 \\
Apolipoprotein B-100 (g/L) & 1.17 & \pm & 0.20 & 1.16 & \pm & 0.17 & -0.01 & \pm & 0.11 & 0.545 \\
\hline
\end{tabular}

Values are means \pm SD

${ }^{1} \mathrm{~N}=45$.

${ }^{2}$ These parameters were tested by a Wilcoxon signed-rank test for non-normal distributed data.

Table 3.4 Effect of 4-wk resveratrol intake on metabolic risk markers ${ }^{3}$

\begin{tabular}{|c|c|c|c|c|c|c|c|c|c|c|}
\hline & \multicolumn{3}{|c|}{ Placebo } & \multicolumn{3}{|c|}{ Resveratrol } & \multicolumn{3}{|c|}{ Difference } & \multirow{2}{*}{$\begin{array}{l}P \text { value } \\
0.760\end{array}$} \\
\hline $\operatorname{BMI}\left(\mathrm{kg} / \mathrm{m}^{2}\right)^{1}$ & 28.3 & \pm & 3.1 & 28.4 & \pm & 3.1 & 0.0 & \pm & 0.5 & \\
\hline Glucose $(\mathrm{mmol} / \mathrm{L})^{1,4}$ & 5.30 & \pm & 0.54 & 5.22 & \pm & 0.49 & -0.08 & \pm & 0.28 & 0.112 \\
\hline Insulin $(\mathrm{mU} / \mathrm{L})^{1}$ & 13.1 & \pm & 5.0 & 12.9 & \pm & 5.3 & -0.2 & \pm & 2.5 & 0.385 \\
\hline $\mathrm{HOMA}_{\mathbb{I R}}{ }^{1,4}$ & 3.13 & \pm & 1.35 & 3.03 & \pm & 1.44 & -0.10 & \pm & 0.69 & 0.208 \\
\hline Systolic BP $(\mathrm{mmHg})^{2,4}$ & 130 & \pm & 18 & 132 & \pm & 17 & 2 & \pm & 15 & 0.234 \\
\hline Diastolic BP $(\mathrm{mmHg})^{2}$ & 84 & \pm & 9 & 86 & \pm & 9 & 2 & \pm & 7 & 0.044 \\
\hline Heart rate $(\mathrm{BPM})^{2}$ & 64 & \pm & 8 & 67 & \pm & 8 & 3 & \pm & 7 & 0.025 \\
\hline $\mathrm{MAP}(\mathrm{mmHg})^{2,4}$ & 99 & \pm & 11 & 102 & \pm & 11 & 2 & \pm & 9 & 0.064 \\
\hline
\end{tabular}

Values are means \pm SD.

${ }^{1} \mathrm{~N}=45,{ }^{2} \mathrm{~N}=44$

${ }^{3} \mathrm{BP}$, blood pressure; BPM, beats per minute; MAP, mean arterial pressure.

${ }^{4}$ These parameters were tested by a Wilcoxon signed-rank test for non-normal distributed data. 
Composition of the diets between the periods was comparable (Table 3.2). Body weight at the end of the control period was $84 \pm 12 \mathrm{~kg}$ and the end of the resveratrol period 84 $\pm 12 \mathrm{~kg}$. These values were not significantly different $(p=0.910)$.

\section{Metabolic risk markers}

The effects of 4-wk resveratrol supplementation on serum lipid and lipoprotein concentrations are presented in Table 3.3. No statistically significant differences were observed between the resveratrol and placebo periods on serum apoA-I and apoB-100 concentrations. In addition, no effects were found on serum total-, LDL- and HDL cholesterol and ratio of total to HDL-cholesterol. Triacylglycerol concentrations were significantly higher $(P<0.05)$ after the resveratrol period compared to the control period.

A significant increase in diastolic blood pressure and heart rate $(P<0.05$ for both variables) was observed after the resveratrol period. Insulin concentrations, HOMA $A_{R}$, systolic blood pressure and MAP were comparable between both periods (Table 3.4).

Table 3.5 shows the effect of resveratrol supplementation on markers for inflammation and endothelial function. No significant differences were found for hsCRP concentrations between both interventions. Also, no differences between both periods were found for IL-6, TNF $\alpha$, E-selectin, thrombomodulin, P-selectin, ICAM-3, sICAM-1, and sVCAM-1 concentrations. Ten subjects ( 6 men and 4 women) had hsCRP levels $>10 \mathrm{mg} / \mathrm{L}$ on one or more occasions, which were equally divided across intervention and time points of blood sampling. Conclusions did not change when these subjects were excluded from analyses.

Table 3.5 Effect of 4-wk resveratrol intake on markers for inflammation and endothelial function ${ }^{1,2}$

\begin{tabular}{lrrrrrrrrrr}
\hline & \multicolumn{3}{c}{ Placebo } & \multicolumn{3}{c}{ Resveratrol } & \multicolumn{3}{c}{ Difference } & P value \\
\hline hsCRP $(\mathrm{mg} / \mathrm{L})^{3}$ & 2.64 & \pm & 2.93 & 4.51 & \pm & 10.24 & 1.87 & \pm & 10.02 & 0.326 \\
IL-6 $(\mathrm{pg} / \mathrm{mL})^{3}$ & 1.63 & \pm & 1.06 & 1.85 & \pm & 1.42 & 0.23 & \pm & 1.26 & 0.718 \\
TNFa $(\mathrm{pg} / \mathrm{mL})^{3}$ & 4.00 & \pm & 1.26 & 3.95 & \pm & 0.74 & -0.05 & \pm & 0.91 & 0.455 \\
E-Selectin $(\mathrm{ng} / \mathrm{mL})^{3}$ & 9.86 & \pm & 6.51 & 9.64 & \pm & 6.72 & -0.22 & \pm & 2.46 & 0.569 \\
Thrombomodulin $(\mathrm{ng} / \mathrm{mL})^{3}$ & 3.32 & \pm & 0.77 & 3.29 & \pm & 0.77 & -0.03 & \pm & 0.34 & 0.815 \\
P-Selectin $(\mathrm{ng} / \mathrm{mL})^{3}$ & 75 & \pm & 68 & 73 & \pm & 52 & -2 & \pm & 82 & 0.467 \\
ICAM-3 $(\mathrm{ng} / \mathrm{mL})^{3}$ & 0.67 & \pm & 0.29 & 0.67 & \pm & 0.30 & 0.00 & \pm & 0.15 & 0.480 \\
sICAM-1 $(\mathrm{ng} / \mathrm{mL})$ & 324 & \pm & 86 & 322 & \pm & 82 & -2 & \pm & 64 & 0.829 \\
sVCAM-1 $(\mathrm{ng} / \mathrm{mL})$ & 561 & \pm & 111 & 570 & \pm & 130 & 8 & \pm & 105 & 0.595 \\
\hline
\end{tabular}

Values are means \pm SD

${ }^{1} \mathrm{~N}=45$.

${ }^{2}$ hsCRP, high-sensitivity C-reactive protein; ICAM-3, intercellular adhesion molecule-3; IL-6, interleukin-6; sICAM-1 soluble intercellular adhesion molecule-1; sVCAM-1, soluble vascular adhesion molecule-1.

${ }^{3}$ These parameters were tested by a Wilcoxon signed-rank test for non-normal distributed data. 
Exploratory data analyses were performed to investigate the possible impact of subjects' characteristics on the results. However, there were no indications that effects were different for men and women, or for obese $\left(B M I \geq 30 \mathrm{~kg} / \mathrm{m}^{2} ; \mathrm{n}=16: 7\right.$ men and 9 women) and non-obese subjects (BMI $<30 \mathrm{~kg} / \mathrm{m}^{2} ; \mathrm{n}=29: 18$ men and 11 women) separately. The same conclusion was drawn, when subgroups were formed based on median levels of day 0 of the control period for the HOMA $A_{\mathbb{R}}$ index, serum triacylglycerol concentrations, or on DHR concentrations or DHR:resveratrol ratios of day 28 of the resveratrol period. When analyses were restricted to subjects that did not use any prescribed medication $(n=31)$, the decrease in plasma glucose concentrations during the resveratrol period became statistically significant $(-0.14 \mathrm{mmol} / \mathrm{L} ; \mathrm{P}<0.05)$

Table 3.6: Effect of 4-wk resveratrol intake on hematologic, hemostatic and general health parameters ${ }^{1,2}$

\begin{tabular}{|c|c|c|c|c|c|c|c|c|c|c|}
\hline \multirow[b]{2}{*}{ Hemoglobin $(\mathrm{mmol} / \mathrm{L})(\mathrm{n}=43)^{3}$} & \multicolumn{3}{|c|}{ Placebo } & \multicolumn{3}{|c|}{ Resveratrol } & \multicolumn{3}{|c|}{ Difference } & \multirow{2}{*}{$\frac{P \text { value }}{0.199}$} \\
\hline & 8.7 & \pm & 0.6 & 8.6 & \pm & 0.6 & -0.1 & \pm & 0.3 & \\
\hline Hematocryte $(L / L)(n=43)^{3}$ & 0.42 & \pm & 0.03 & 0.42 & \pm & 0.03 & 0.00 & \pm & 0.02 & 0.239 \\
\hline Erytrocytes $\left(10^{12} / \mathrm{L}\right)(\mathrm{n}=42)^{3}$ & 4.61 & \pm & 0.29 & 4.58 & \pm & 0.29 & -0.02 & \pm & 0.18 & 0.568 \\
\hline $\operatorname{MCV}(f L)(n=43)^{3}$ & 91.3 & \pm & 3.7 & 91.1 & \pm & 3.5 & -0.1 & \pm & 1.2 & 0.484 \\
\hline $\mathrm{MCH}(\mathrm{fmol})(\mathrm{n}=44)^{3}$ & 1.84 & \pm & 0.30 & 1.88 & \pm & 0.10 & 0.04 & \pm & 0.27 & 0.744 \\
\hline $\mathrm{MCHC}(\mathrm{mmol} / \mathrm{L})(\mathrm{n}=43)$ & 20.6 & \pm & 0.5 & 20.6 & \pm & 0.5 & 0.0 & \pm & 0.4 & 0.821 \\
\hline Thrombocytes $\left(10^{9} / L\right)(n=43)^{3}$ & 237 & \pm & 49 & 240 & \pm & 49 & 1.4 & \pm & 24 & 0.651 \\
\hline $\operatorname{RDW}(\%)(n=39)^{3}$ & 13.5 & \pm & 0.6 & 13.4 & \pm & 0.6 & -0.1 & \pm & 0.41 & 0.693 \\
\hline Leucocytes $\left(10^{9} / L\right)(n=43)$ & 5.5 & \pm & 1.2 & 5.5 & \pm & 1.4 & 0.0 & \pm & 0.9 & 0.944 \\
\hline PT $(s)(n=31)^{3}$ & 11.1 & \pm & 1.4 & 11.5 & \pm & 4.1 & 0.5 & \pm & 3.0 & 0.904 \\
\hline $\operatorname{aPTT}(s)(n=32)^{3}$ & 27.8 & \pm & 1.9 & 27.5 & \pm & 1.8 & 0.1 & \pm & 1.2 & 0.664 \\
\hline Sodium (mmol/L) & 142 & \pm & 1.8 & 142 & \pm & 1.4 & -0.1 & \pm & 1.9 & 0.841 \\
\hline Potassium $(\mathrm{mmol} / \mathrm{L})^{3}$ & 4.31 & \pm & 0.25 & 4.32 & \pm & 0.29 & 0.01 & \pm & 0.30 & 0.535 \\
\hline Calcium (mmol/L) & 2.31 & \pm & 0.06 & 2.31 & \pm & 0.08 & 0.00 & \pm & 0.06 & 0.850 \\
\hline Phosphorus (mmol/L) & 1.05 & \pm & 0.14 & 1.03 & \pm & 0.13 & -0.02 & \pm & 0.09 & 0.112 \\
\hline Chloride (mmol/L) & 104 & \pm & 3.5 & 103 & \pm & 3.4 & -0.2 & \pm & 2.3 & 0.568 \\
\hline Ureum (mmol/L) & 5.0 & \pm & 1.2 & 4.9 & \pm & 1.4 & -0.1 & \pm & 0.9 & 0.320 \\
\hline Creatinin (mmol/L) & 77.7 & \pm & 11.7 & 77.3 & \pm & 11.5 & -0.4 & \pm & 5.7 & 0.640 \\
\hline $\operatorname{ALP}(U / L)^{3}$ & 81.8 & \pm & 19.4 & 85.8 & \pm & 22.6 & 4.0 & \pm & 9.4 & 0.009 \\
\hline GGT $(U / L)^{3}$ & 29.1 & \pm & 18.9 & 28.7 & \pm & 16.7 & -0.4 & \pm & 12.7 & 0.955 \\
\hline $\operatorname{ASAT}(\mathrm{U} / \mathrm{L})^{3}$ & 23.6 & \pm & 11.0 & 24.8 & \pm & 9.8 & 1.2 & \pm & 5.8 & 0.308 \\
\hline $\operatorname{ALAT}(U / L)^{3}$ & 27.1 & \pm & 25.6 & 25.4 & \pm & 16.8 & -1.7 & \pm & 17.4 & 0.567 \\
\hline Total bilirubin $(\mu \mathrm{mol} / \mathrm{L})^{3}$ & 10.1 & \pm & 5.4 & 10.3 & \pm & 6.1 & 0.2 & \pm & 3.0 & 0.982 \\
\hline Total protein (g/L) & 70.8 & \pm & 3.0 & 70.7 & \pm & 3.6 & -0.1 & \pm & 2.7 & 0.884 \\
\hline Albumin $(\mathrm{g} / \mathrm{L})^{3}$ & 43.1 & \pm & 3.0 & 43.3 & \pm & 3.1 & 0.1 & \pm & 2.0 & 0.559 \\
\hline
\end{tabular}

Values are means \pm SD

${ }^{1} \mathrm{~N}=45$, unless otherwise indicated.

${ }^{2}$ ALAT, alanine aminotransferase; ALP, alkaline phosphatase; aPTT, activated partial thromboplastin time; AST, aspartate aminotransferase; GGT, $\gamma$-glutamyl transpeptidase; $\mathrm{MCH}$, mean corpuscular hemoglobin; $\mathrm{MCHC}$, mean corpuscular hemoglobin concentration; MCV, mean corpuscular volume; PT, prothrombin time; RDW, relative distribution weight

${ }^{3}$ These parameters were tested by a Wilcoxon signed-rank test for non-normal distributed data. 


\section{General health parameters}

Apart from statistically significant higher ALP values after resveratrol supplementation, no differences were found after 4-wk resveratrol treatment compared to control in any of the clinical chemistry, hematology and coagulation parameters (Table 3.6).

\section{Discussion}

In this study with overweight and slightly obese men and women, we found no effects on serum apoA-I and other metabolic risk markers after 4-wk of daily supplementation with $150 \mathrm{mg}$ trans-resveratrol. Compliance of the subjects was excellent, as indicated by capsule counts and total plasma resveratrol concentrations. A lack of effect on apoA-I concentrations does not support in vitro data, which suggests that resveratrol induces the expression of the apoA-I gene $(83,92)$. So far, however, no human studies have specifically addressed the effects of trans-resveratrol on apoA-I concentrations. Our finding that resveratrol supplementation also has no effect on HDL cholesterol agrees with the results of a very recent meta-analysis. In that study, no effects of resveratrol on HDL cholesterol concentrations were found, irrespective of dose, study duration, and cardiovascular risk status of the subjects (61). A major limitation of this meta-analysis was that, in four out of the seven studies included, subjects used cholesterol-lowering and/or hypoglycemic drugs, while in none of the studies changes in serum lipid concentrations were the primary outcome parameter. In our study, however, subjects did not use these types of medication and were selected based on low HDL cholesterol concentrations. In addition, in only five of the seven studies, purified trans-resveratrol was used, whereas in the other studies resveratrol-enriched grape extract or Polygonum Cuspidatum extract containing trans-resveratrol was given. However, like in the recent meta-analysis, we also did not observe any effects on serum total cholesterol, LDL cholesterol and in addition apoB100 concentrations. Another recent double-blind cross over trial tested the effects of high-dose (e.g. $1000 \mathrm{mg}$ during the first week and 2000 mg during the second week) trans-resveratrol intake on apoB100 production- and catabolic rate in overweight and obese, mildly hypertriglyceridemic males. Reductions of both apoB100 production and catabolic rates were found without accompanying changes in plasma triglyceride-rich lipoprotein (TRL) apoB100 concentrations (93). This may agree with our results, although it should be noted that the major of the plasma apoB100 pool is transported by the triglyceride-poor LDL particles. To summarize, it is not very likely that the putative positive effects of resveratrol on cardiovascular health are mediated by changes in serum lipid or lipoprotein concentrations.

The question then remains whether resveratrol protects against cardiovascular disease through effects other than those on lipid and lipoprotein metabolism. Several studies have reported positive effects of resveratrol on insulin sensitivity, accompanied 
by a significant lowering of blood glucose levels. However, we did not observe a statistically significant change in $\mathrm{HOMA}_{\mathrm{IR}}$ and a tendency towards decreased fasting plasma glucose concentrations, which became statistically significant, when subjects who used medication were excluded from the statistical analyses. Results of resveratrol on plasma glucose concentrations are conflicting, and both positive and no-effect studies have been reported. Again, studies have been performed in diverse populations. Effects on fasting glucose concentrations have mainly been found in studies with type 2 diabetes mellitus (T2DM) patients $(94,95)$, although part of these results have to be interpreted cautiously because of methodological constraints (95). No effects have been found in a study that included obese, but otherwise healthy subjects (87). Two other studies used the same type of trans-resveratrol as we did $(88,89)$. In one study, no effect on fasting glucose concentrations was found after 12 weeks of $75 \mathrm{mg} / \mathrm{d}$ resveratrol supplementation in non-obese women with normal glucose tolerance (89), whereas Timmers et al. reported a significant decrease of $0.18 \mathrm{mmol} / \mathrm{L}$ in glucose after 30-day resveratrol supplementation in obese, but healthy male subjects (88). Although changes in glucose concentrations were a secondary outcome, our study had a statistical power of nearly $100 \%$ to detect a true change in glucose of $0.18 \mathrm{mmol} / \mathrm{L}$ at an alpha of 0.05. Thus, daily supplementation with $150 \mathrm{mg}$ trans-resveratrol may improve glucose metabolism, but more well-designed studies are needed to answer this question.

In vitro and animal studies have suggested positive effects of resveratrol on endothelial function and low-grade systemic inflammation (96). However, our results are in line with other human studies that did not show an effect on different markers for low-grade inflammation, such as CRP, IL1 $\beta$, IL6, IL8, TNF $\alpha$ and monocyte chemotactic protein-1 (87$89,94,97)$. In one study, a reduction in TNFa (88) was found, which we - and others (87) - could not reproduce. Regarding vascular and endothelial function, Agarwal et al. showed a lower expression of VCAM- and ICAM mRNA after treatment of human coronary artery endothelial cells with plasma from subjects treated with resveratrol (97). However, we found no effects on circulating levels of ICAM-3, SICAM-1, sVCAM-1, ESelectin and P-Selectin. Therefore, consistent evidence from human studies for beneficial effects of resveratrol intake on plasma markers for low-grade inflammation and endothelial function is missing.

Finally, it has been suggested that trans-resveratrol lowers systolic blood pressure (SBP) and mean arterial pressure (MAP) (88). However, these effects could not be confirmed in other studies, which also found no effects on diastolic blood pressure (DBP) and heart rate $(87,89,98)$. In our study, SBP and MAP also remained unchanged, but DBP and heart rate increased after trans-resveratrol intake, for which we have no explanation.

It is possible that certain groups may benefit from resveratrol supplementation and other groups do not. Although our study was not powered to answer this question 
adequately, exploratory data analyses did not suggest that effects did depend on sex, $\mathrm{BMI}$, triacylglycerol concentrations or $\mathrm{HOMA}_{\mathrm{IR}}$. Interestingly, Bode et al. showed pronounced inter-individual differences in trans-resveratrol metabolism by human gut microbiota (99), which might also contribute to discrepancies between studies. Also, in our subjects we found large differences in plasma DHR concentrations. However, we found no indications that results did depend on DHR concentrations or DHR:resveratrol ratios.

In summary, we here show that $150 \mathrm{mg}$ of daily resveratrol intake for four weeks does not change lipids, lipoproteins and other metabolic risk markers related to cardiovascular health in overweight and slightly obese men and women with low HDL cholesterol concentrations. Effects on glucose metabolism however warrant further study.

\section{Acknowledgments}

We would like to thank Martine Hulsbosch and Maud Beckers for their technical support and Nina Wystyrk for her dietary assistance. RPM, SMM and JP designed research; SMM conducted research; SMM and RPM analyzed data; SMM, RPM and JP and wrote paper; RPM had primary responsibility for final content. All authors read and approved the final manuscript. Neither of the authors had a conflict of interest. 



\title{
CHAPTER 4
}

\author{
Resveratrol does not affect markers for \\ endothelial function and arterial flexibility in \\ the fasting or postprandial phase: a \\ randomized, placebo-controlled crossover \\ trial
}




\section{Abstract}

Background Studies on the effects of longer-term intake of trans-resveratrol on flowmediated vasodilation (FMD) are conflicting. In addition, effects of longer-term transresveratrol intake on endothelial function during the postprandial phase are not known. Objective: To study in overweight and slightly obese subjects the effect of longer-term trans-resveratrol intake on FMD. Furthermore, effects on arterial stiffness, as measured by pulse wave velocity (PWV) were assessed, as well as effects on vascular function after the intake of a mixed meal.

Design A randomized, placebo-controlled crossover study was conducted in 45 overweight and slightly obese men $(n=25)$ and women $(n=20)$ with a mean age of $61 \pm$ 7 years. Subjects received in random order resveratrol (150 $\mathrm{mg}$ per day) or placebo capsules for 4 weeks, separated by a 4-week wash-out period. At the end of each intervention period, FMD and PWV measurements were performed before and after a mixed meal challenge.

Results Compared with placebo, resveratrol had no effects on endothelial function. After resveratrol supplementation, fasting FMD was $0.1 \%$ lower ( $P=0.692)$, and PWV was $0.6 \mathrm{~m} / \mathrm{s}$ higher $(\mathrm{P}=0.159)$. These parameters were also not differently affected after the postprandial test. Also, biomarkers for endothelial function (sE-selectin, sICAM-3, sPselectin, sThrombomodulin, sVCAM-1), inflammation (IL-6, TNFa), glucose and lipid metabolism were comparable during the postprandial tests.

Conclusion $150 \mathrm{mg}$ of daily resveratrol intake does not change endothelial function and markers for endothelial activation and inflammation in the fasting state or postprandial phase in overweight and slightly obese men and women. 


\section{Introduction}

It has been suggested that resveratrol, a plant polyphenolic compound with antioxidant activity found in for example the skin of black grapes -the average trans-resveratrol content of red wines is $1.9 \mathrm{mg} / \mathrm{L}$, varying from non-detectable levels up to $14.3 \mathrm{mg} / \mathrm{L}$ (100)-, has beneficial health effects (101). However, we have earlier reported that in overweight and slightly obese men and women a daily supplement of $150 \mathrm{mg}$ transresveratrol for four weeks did not improve fasting cardiovascular metabolic risk markers (102). Actually, human data on the effects of resveratrol on metabolic risk is limited and results are conflicting (87-89). Nonetheless, resveratrol might affect other parameters related to cardiovascular health, such as endothelial function. In vitro evidence for beneficial effects of resveratrol on endothelial function was provided by Wallerath and colleagues, who demonstrated that resveratrol up-regulated endothelial nitrogen oxide synthase (eNOS) mRNA and protein expression, and nitrogen oxide (NO) production in a time- and concentration-dependent manner in human umbilical vein endothelial cells (HUVECs) (103). A more recent study extended these findings by stimulating HUVECs with physiologically more relevant resveratrol concentrations and pointed towards other pathways that might underlie the potential positive effects of resveratrol on endothelial function such as sirtuin 1 (SIRT1) upregulation and cyclo-oxygenase 1 (COX-1) inhibition (104). In humans, endothelial function can non-invasively be assessed by flow-mediated dilation (FMD) (17). Wong et al. were the first to suggest that FMD improved dosedependently after the acute intake of 30,90 or $270 \mathrm{mg}$ trans-resveratrol in overweight and obese adults with elevated blood pressure (105). The same group also showed an improvement in FMD after longer-term intake of $75 \mathrm{mg}$ trans-resveratrol daily for 6 weeks in obese, but otherwise healthy adults (98). However, in another study no effect on FMD was observed in 40 post-infarction patients after the intake of $10 \mathrm{mg}$ transresveratrol daily for 3 months (106). Effects of longer-term trans-resveratrol intake on endothelial function during the postprandial phase have not been studied $(107,108)$. Introducing a high-fat dietary challenge negatively influences whole body metabolism, possibly through increasing the inflammatory response and oxidative stress, which may reduce vascular function $(44,45)$. Hence, such a dietary challenge may enable us to evaluate subtle changes in low-grade systemic inflammation, endothelial function and postprandial metabolism following a resveratrol intervention. Therefore, the objective of the present study was to investigate whether 4 weeks intake of $150 \mathrm{mg}$ resveratrol daily improves endothelial function and markers for low-grade systemic inflammation in the fasting state and during the postprandial phase in overweight and slightly obese subjects. 


\section{Subjects and methods}

\section{Subjects and study design}

Overweight and obese healthy subjects with a mean age of $61 \pm 7$ years participated in a double-blind, randomized, placebo-controlled, cross-over study in which they either consumed resveratrol (150 mg/day; resVida ${ }^{\mathrm{TM}}$, 99.9\% trans-resveratrol) or placebo capsules for 4 weeks (provided by DSM Nutritional Products Ltd., Kaiseraugust, Switzerland). The two treatment periods were separated by a wash-out period of at least 4 weeks. Inclusion and exclusion criteria have been described before (102). Briefly, all subjects were apparently healthy, did not suffer from cardiovascular diseases. Further, they were selected based on decreased high-density lipoprotein (HDL) cholesterol concentrations, determined during two screening visits, as the primary aim was to study the effects of resveratrol intake on serum apoA-I concentrations. The inclusion criterion for $\mathrm{HDL}$ cholesterol concentrations was $<1.21 \mathrm{mmol} / \mathrm{L}$ for men and $<1.53 \mathrm{mmol} / \mathrm{L}$ for women. These cut-off values were the mean values found in the PROCAM study (32).

At the end of each 4-wk period, a postprandial test started in the morning after a $12 \mathrm{~h}$ overnight fast. Subjects were asked to refrain from any strenuous physical exercise and not to consume alcohol on the day before testing. Before and 240 minutes after the postprandial test, FMD and arterial stiffness were measured. After the vascular measurements in the fasting state, an intravenous cannula (Becton, Dickinson and Company, Franklin Lanes, NY, USA) was inserted in an antecubital vein and a fasting blood sample (TO) was collected. Participants were then asked to consume a high-fat test meal within $10 \mathrm{~min}$. Subsequent blood samples were drawn 15 (T15), 30 (T30), 45 (T45), 60 (T60), 90 (T90), 120 (T120), 180 (T180) and 240 (T240) min after meal consumption. Subjects were allowed to drink water at their convenience throughout the postprandial test.

\section{Test meal}

During each postprandial test, subjects received two muffins and $300 \mathrm{~mL}$ low-fat milk ( $0 \%$ vet Melkdrank, Campina, Friesland-Campina, The Netherlands). The meal provided $4598 \mathrm{~kJ}, 26.5 \mathrm{~g}$ protein, $121 \mathrm{~g}$ carbohydrates and $56.6 \mathrm{~g}$ fat (Table 4.1, fat/carbohydrate/protein: 28 en\%/59 en\%/13 en\%). One batch of muffins was prepared for the entire study and stored at $-20^{\circ} \mathrm{C}$ until consumption. 


\section{Flow-mediated dilation}

Endothelium-dependent vascular reactivity was measured as FMD of the brachial artery in accordance with recommendations stated by the International Brachial Artery Reactivity Task Force (17). The same trained ultrasonographer performed both measurements at the end of both periods in all subjects after at least 15-min supine rest. For 28 subjects, all FMD measurements were made with a high-resolution ultrasound system using a 3-11 MHz wide band linear array transducer (Philips Sonos 5500 system; Philips Ultrasound, Andover, MA, USA). For 13 subjects, measurements were performed with a 5-10 Mhz linear array probe (Picus I, Esaote Biomedica, Genoa, Italy). Baseline FMD measurements were comparable between both systems, as assessed by an independent student's T-test $(\mathrm{P}=0.921$ after control period, $\mathrm{P}=0.729$ after experimental period). Images of the brachial artery were taken longitudinally, 2-10 cm above the antecubital fossa in combined Doppler / brightness mode (B-mode). A probe holder was used to secure the position of the transducer during the measurement. After a 3 min baseline measurement, a peripherally hypoxic state was induced by inflation of a pneumatic tourniquet (Hokanson TD312 automatic cuff inflator, D.E. Hokanson, Inc. WA, USA) placed around the forearm, inflated to a pressure of at least $50 \mathrm{mmHg}$ above systolic pressure, with a minimal pressure of $200 \mathrm{mmHg}$. After 5 minutes, the cuff was deflated, resulting in reactive hyperemia, which was recorded for another 5 minutes. Images were continuously recorded on DVD during the entire $13 \mathrm{~min}$ measurement protocol. Acquired images were analyzed using an in-house developed semi-automated image-analysis algorithm (Prof. A.P. Hoeks, Department of Biomedical Engineering, Maastricht University Medical Center, The Netherlands). FMD responses were expressed as the percentage increase in brachial artery diameter from baseline to maximal dilation post-occlusion.

\section{Arterial Stiffness}

Arterial stiffness was assessed by carotid-femoral pulse wave velocity ( $\left.P W V_{c-f}\right)$ measurements (SphygmoCor CPV System, AtCor Medical Pty. Ltd., West Ryde, Australia). $P W V_{c-f}$ was computed from the time delay between the upstroke of the arterial pressure wave measured at the femoral and carotid artery. Distance between measuring sites was assessed by tape measure in a straight line. $80 \%$ of this distance was used for calculations, according to the consensus paper by van Bortel et al. (26). Measurements were made after at least 30-min supine rest in a quiet, temperature-controlled, darkened room. An automated sphygmomanometer (Mobilograph NG, IEM GmbH, Stolberg, Germany) was used to measure supine blood pressure. Peripheral and central augmentation indexes (Alx $x_{p}$ and $A x_{c}$ respectively) were measured at the radial site by tonometry (SphygmoCor CPV System, AtCor Medical Pty. Ltd., West Ryde, Australia). Central aortic pressure waveforms were derived automatically from the radial waveform 
using a validated generalized transfer function by the software. Augmentation index was calculated as the difference in pressure between the first peak or shoulder and the second peak or shoulder of the waveform (augmentation pressure) divided by the difference in maximal central systolic and diastolic pressure (central pulse pressure). Arterial stiffness and FMD measurements were performed at baseline and 4 hours postprandially at the end of the experimental and control periods (day 28 of each 4 week period).

\section{Blood analyses}

Blood was sampled in EDTA-containing, NaF-containing and serum separator tubes (Becton, Dickinson and Company, Franklin Lanes, NY, USA). Blood drawn in serum separator tubes was allowed to clot for at least $30 \mathrm{~min}$ at $21^{\circ} \mathrm{C}$, followed by centrifugation at $1300 \times \mathrm{g}$ for $15 \mathrm{~min}$ at $21^{\circ} \mathrm{C}$. NaF- and EDTA-containing tubes were immediately placed on ice after blood drawing. Tubes were centrifuged at $1300 \mathrm{x} g$ for $15 \mathrm{~min}$ at $4^{\circ} \mathrm{C}$. Plasma and serum aliquots were directly snap-frozen in liquid nitrogen and stored at $-80^{\circ} \mathrm{C}$ until analysis.

Plasma glucose concentrations were measured in NaF plasma at T0, 15, 30, 45, 60, 90, 120 and 240 (Horiba ABX, Montpellier, France). Concentrations of TAG, corrected for free glycerol, were determined in serum samples from T0, 60, 120, 180 and 240 (GPO Trinder; Sigma Diagnostics). Insulin concentrations were measured in EDTA plasma at T0, 15, 30, 45, 60, 90, 120, 180 and 240 (RIA; Millipore, Billerica, MA, USA). IL-6, TNF $\alpha$, sEselectin, soluble thrombomodulin, sP-selectin, sICAM-3, sICAM-1 and sVCAM-1 were measured in EDTA plasma at T0, 120 and 240 by commercially available Multi Spot ELISA kits (Meso Scale Discovery, Rockville, MD, USA).

\section{Statistical analyses}

Data are presented as mean \pm SD, unless otherwise indicated. Fasting concentrations between the experimental and control period, as well as changes within a group, were compared using a paired T-test. Overall responses of glucose, insulin and TAG were evaluated by calculating the incremental areas under the postprandial curve (iAUCs) using the trapezoidal rule (109). Maximal changes in concentration were calculated by subtracting baseline values from maximal or minimal value. Differences in iAUC and maximal changes between the experimental and control periods were tested for significance by a paired T-test.

Changes in concentrations over time for glucose, insulin, TAG, sE-selectin, sICAM-1, sICAM-3, IL-6, sP-Selectin, soluble thrombomodulin, TNF $\alpha$ and SVCAM were tested by linear mixed models, with diet and time as fixed factors and diet $x$ time as interaction 
term. If the term was not statistically significant, it was omitted from the model. If factor time was significant, post hoc tests with Bonferroni correction were carried out to compare concentrations to baseline concentrations.

For PWV and FMD, changes between baseline and postprandial values were calculated. Differences between the experimental and control periods were evaluated for baseline measurements and postprandial changes by a student's paired T-test. Results were considered to be statistically significant if $P<0.05$. Statistical analyses were performed using SPSS 19.0 for Mac Os X (SPSS Inc., Chicago, IL, USA).

\section{Results}

\section{Study participants}

The flow of participants through the study is shown in Figure 3.1. A total of 45 subjects finished the study. Baseline characteristics of these subjects are shown in Table 4.2. Due to technical problems, we were unable to perform FMD measurements in two subjects on one test-day. For six subjects, one measurement could not be interpreted. Therefore, FMD results are shown for a total of 37 subjects.

\section{Vascular function measurements}

Fasting FMD values at the end of the resveratrol and control periods were not significantly different. In addition, changes in FMD during the postprandial phase were not different between both interventions. Within the control group, we also did not observe an effect on FMD of the mixed meal challenge $(P=0.57)$. Also, no differences were found between fasting arterial diameters, and the difference in postprandial changes after the experimental and control period. Furthermore, measures for arterial stiffness were not affected by resveratrol intake, as fasting values and postprandial changes after meal intake for $\mathrm{PWV}_{\mathrm{cf}}$, peripheral augmentation index $\left(A \mid \mathrm{x}_{\mathrm{p}}\right)$, central $\mathrm{Alx}$ $\left(A \mid x_{c}\right)$ and central Alx adjusted for a heart rate corrected to 75 beats per minute (Alx $\left.x_{c} H R 75\right)$ were comparable between experimental and control period (Table 4.3). 


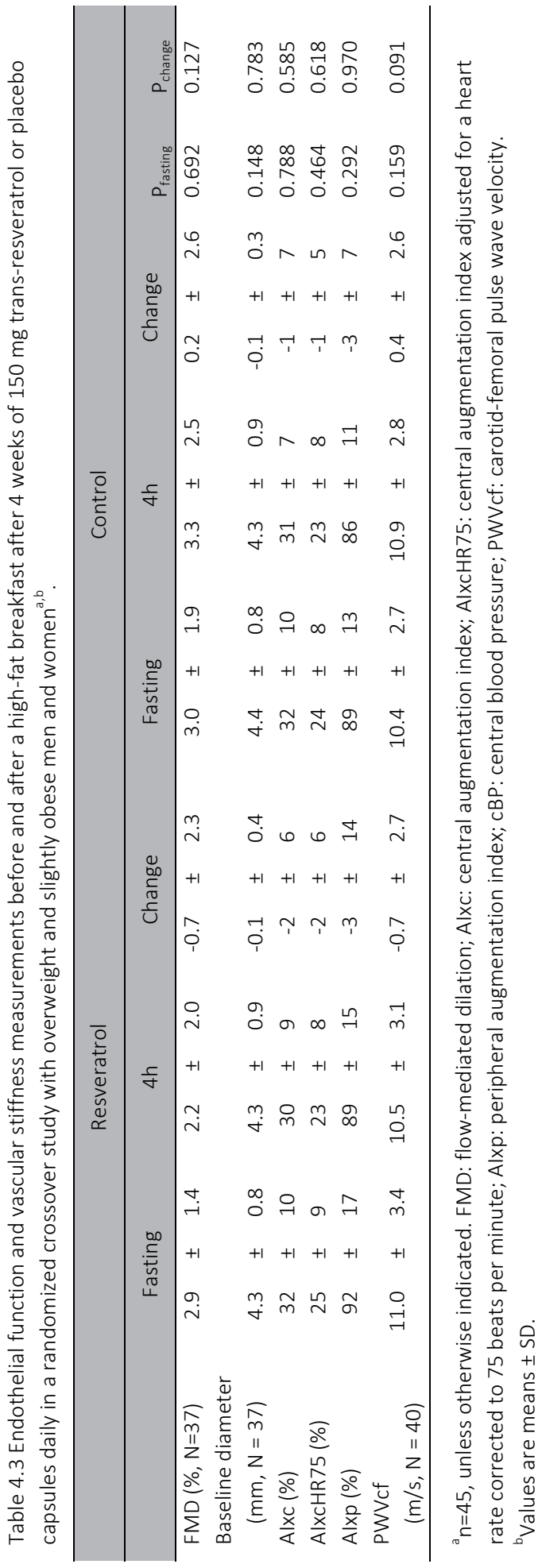




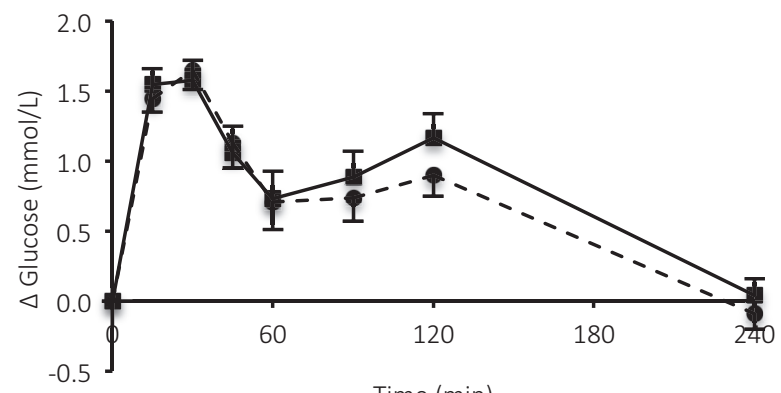

Time (min)
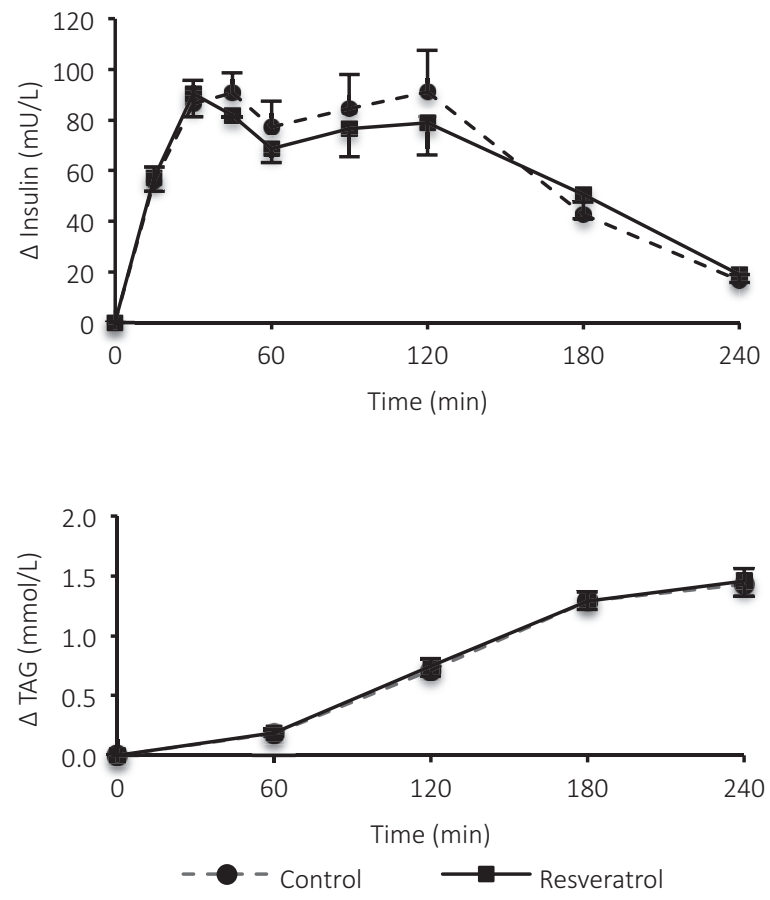

Figure 4.1 Mean changes \pm SEM in plasma glucose and serum insulin and triacylglycerol (TAG) concentrations following a mixed meal postprandial challenge after control $(\bullet)$ and resveratrol ( $\mathbf{\square})$ period in a randomized crossover study with overweight and obese men and women $(\mathrm{N}=45)$. Data were analysed using Linear Mixed Models. The increase in TAG, glucose and insulin concentrations were significant for the factor time $(P<0.001)$. There were no significant diet $x$ time interactions $(P>0.05)$. 


\section{Postprandial glycemia and lipemia}

Fasting concentrations of glucose, insulin and triacylglycerol (TAG) did not differ between the test days at the end of the control and experimental periods. Glucose and insulin concentrations significantly increased after mixed meal consumption $(P<0.001$ for time effect). This increase, however, was not significantly different between control and experimental periods. Also, the iAUCs for glucose and insulin were comparable between control and experimental periods. TAG concentrations increased significantly after the mixed meal $(P<0.001$ for time effect). However, no effects of resveratrol were found (Figure 4.1).

\section{Postprandial markers of endothelial activation and inflammation}

Fasting concentrations of markers for endothelial activation (sE-Selectin, sICAM-1, sICAM-3, sP-Selectin, soluble thrombomodulin, sVCAM) and inflammation (IL-6, TNF $\alpha$ ) were comparable between the experimental and control period. No significant interactions between intervention and time were found, except for sICAM-1 ( $P=0.02)$. At T120, sICAM-1 concentrations were higher after consuming resveratrol for 4 weeks ( $P$ $=0.04)$. For all other markers, time effects were significant $(P<0.001$, Figure 4.2).

\section{Subgroup analyses}

Exploratory data analyses were performed to explore the possible impact of subjects' characteristics on the results. However, no indications were found for different effects based on sex, degree of obesity (BMI $\geq 30 \mathrm{~kg} / \mathrm{m}^{2} ; \mathrm{n}=16: 7$ men and 9 women versus $\mathrm{BMI}<30 \mathrm{~kg} / \mathrm{m}^{2} ; \mathrm{n}=29: 18$ men and 11 women) high-sensitivity C-reactive protein concentrations (hsCRP) or when subgroups were formed based on dihydroresveratrol (DHR) concentrations or DHR:resveratrol ratios of day 28 of the control period (both hSCRP and DHR were measured as described before (102). Finally, when analyses were restricted to subjects that did not use any prescribed medication $(n=31)$, conclusions were also comparable. 

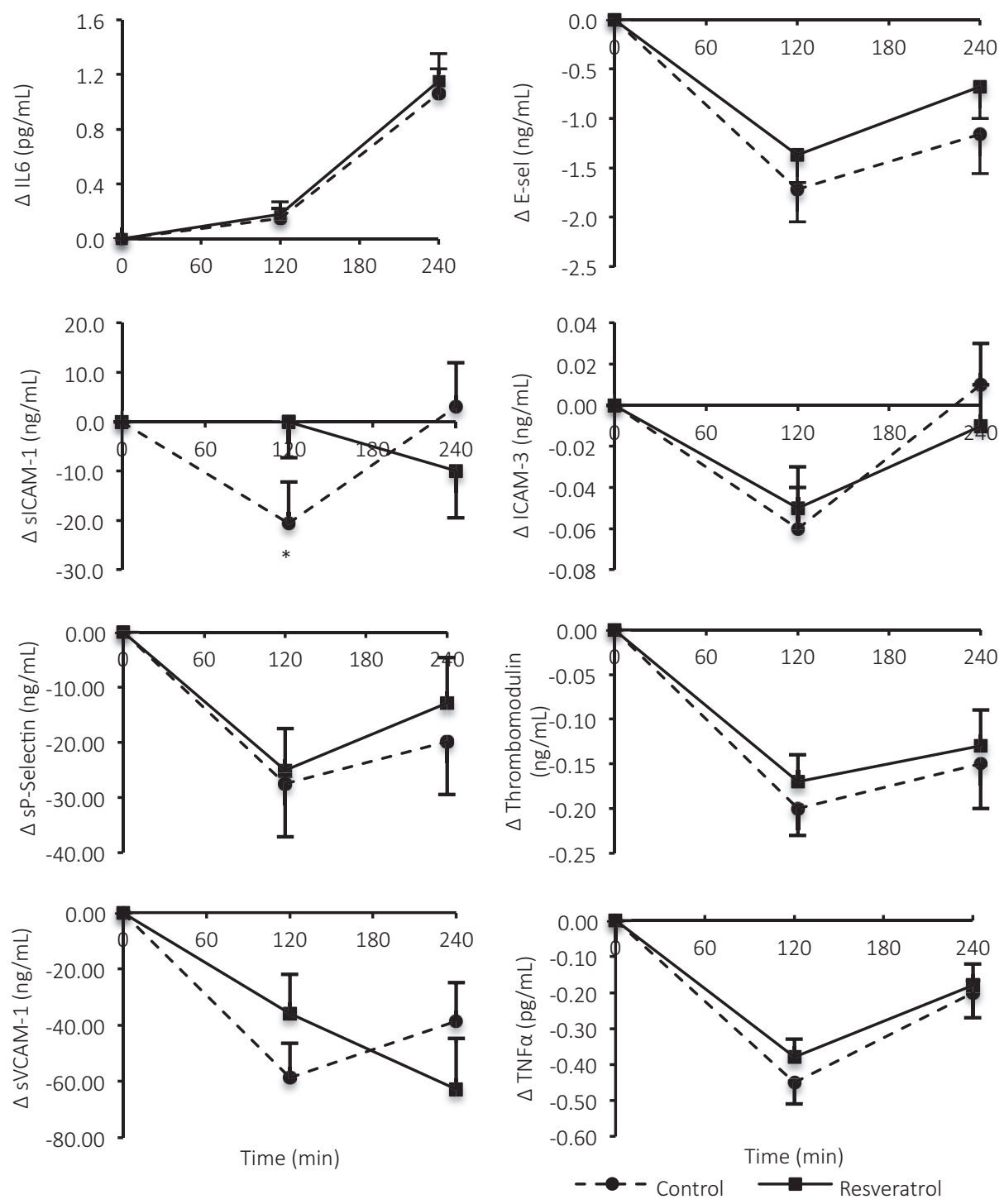

Figure 4.2 Mean ( \pm SEM) changes in IL-6, sE-Selectin, sICAM-1, sICAM-3, sP-Selectin, sThrombomodulin, sVCAM-1 and TNF $\alpha$ concentrations after a mixed meal following the control $(\bullet)$ and resveratrol $(\boldsymbol{\square})$ period in a randomized crossover study with overweight and obese men and women $(\mathrm{N}=45)$. Data were analysed using Linear Mixed Models. The increase in IL6 and decrease in sE-selectin, sICAM-3, sP-selectin, sThrombomodulin, sVCAM-1 and TNF $\alpha$ concentrations were significant for the factor time $(P<0.001)$. There were no significant diet $x$ time interactions $(P>0.05)$.

*significant difference between experimental and control period $(P<0.05)$ 


\section{Discussion}

We found that in overweight and slightly obese men and women the intake of $150 \mathrm{mg}$ trans-resveratrol daily for four weeks had no effect on FMD, arterial stiffness and plasma markers for endothelial function and low-grade inflammation in fasting conditions and during the postprandial phase.

In vitro and animal studies have found positive effects of resveratrol on endothelial function through upregulating activity and expression of eNOS, increasing NO bioavailability, upregulation of SIRT1, and inhibition of COX-1 $(103,104,110,111)$. However, resveratrol undergoes fast and extensive metabolic conversion, leading to low plasma resveratrol concentrations and high plasma concentrations of resveratrol metabolites, such as resveratrol monosulfate or dihydroresveratrol monoglucuronide (112). We also found that plasma total dihydroresveratrol concentrations were about twice as high as those for total resveratrol (102). Whether this indicates that resveratrol metabolites are responsible for the in vivo effects observed in animal studies instead of resveratrol itself warrants further study. Also in humans, positive effects of resveratrol supplementation on endothelial function as measured with FMD have been reported. In an acute study in 19 overweight and obese adults with an elevated blood pressure, Wong et al. showed a dose-dependent effect of trans-resveratrol intake (0, 30, 90, 270 $\mathrm{mg}$ ) on FMD, as measured 1 hour after consumption (105). In a subsequent 6-wk study in 28 obese adults, but otherwise healthy adults (BMI $30-45 \mathrm{~kg} / \mathrm{m}^{2}$ ), a daily supplement of $75 \mathrm{mg}$ of trans-resveratrol increased the FMD by $1.38 \%$ from $5.83 \%$ with placebo supplementation compared to $7.21 \%$ with resveratrol supplementation (98). However, we found no effects of resveratrol supplementation on FMD. We had FMD data available for 37 subjects, leading in retrospect to a statistical power of $>95 \%$ to detect a true change in FMD of $1.38 \%$ at an alpha of 0.05 . Thus, our study was certainly powered to detect these effects as well. Although our subjects had a lower BMI, exploratory subgroup analyses also did not indicate an effect on FMD in obese subjects. It should be noted, however, that the statistical power for subgroup analyses might not have been sufficient. It is also possible that the duration of our study was not long enough, as the study by Wong et al. lasted 6 weeks. Finally, FMD values in our study were lower than those measured by Wong et al. (98), although it is difficult to compare absolute FMD values between research groups. However, it is possible that endothelial function of our subjects was too poor and therefore not responsive to dietary changes within 4 weeks. Alternatively, it can be reasoned that a lower FMD can more easily be improved. Thus, we cannot exclude that study duration and differences in baseline characteristics of the subjects may explain the differences in findings between our study and the study of Wong et al (98). 
Except for FMD measurements in the fasting state, participants received a mixedmeal challenge to examine whether the intake of $150 \mathrm{mg}$ trans-resveratrol for 4 weeks could modify the postprandial FMD response. To disentangle the effects of longer-term resveratrol supplementation from those of an acute challenge, we deliberately chose not to incorporate any resveratrol in the mixed meal. However, resveratrol did not modify postprandial changes in FMD, while we did observe the expected increase in postprandial TAG concentrations that may deteriorate FMD 4-hr postprandially (113). We did however not observe a change in postprandial FMD during the control period. An earlier study with 21 overweight and slightly obese men performed by our group showed an impaired FMD two hours after consuming a high fat meal, which was identical to the one used in the present study. This postprandial FMD impairment was improved after consuming $140 \mathrm{~mL}$ beetroot juice (114). In the present study, FMD was measured 4 hours postprandially instead of the 2 hours in the beetroot juice study, which may suggest that FMD had already returned to fasting values. However, other studies did show a 4-hour postprandial deterioration of FMD after a high-fat meal (115, 116). As no differences were found on postprandial glucose, insulin and TAG concentrations between the control and intervention periods, the absence of effects on FMD was not confounded by differences in glucose or lipid metabolism between the experimental and control period.

Finally, resveratrol supplementation did not affect PWV and PWA, in the fasting or in the postprandial phase. Wong et al. also found no effects of resveratrol on arterial compliance in their 6-wk trial (98). To the best of our knowledge, no other studies assessed the effect of pure resveratrol on PWV. However, four weeks of pomegranate juice supplementation, which is a source of resveratrol, also had no effect on PWV (117). Dark chocolate, however, which is rich in flavanols, improved fasted PWV within four weeks, while blood pressure did not change (118). This suggests that the timeframe chosen in our study was not too short to demonstrate changes in PWV.

Anti-inflammatory effects of resveratrol have also been reported. Treating human coronary arterial endothelial cells with resveratrol at nutritionally relevant concentrations inhibited TNFa induced NFKB activity (96). Also, inflammatory gene expression was inhibited in vitro (119) and a decrease in monocyte adhesiveness to the endothelium was observed in human saphenous vein endothelial cells (120) after resveratrol treatment. In the present study, no effects were found on serum hsCRP, TNF $\alpha, I L-6$, sICAM-1, sICAM3 and SVCAM concentrations. Others did find a decrease in TNF $\alpha$, but no effects on CRP, IL-1 $\beta, I L-6$ and IL-8 after 30-day 150 mg trans-resveratrol treatment (88). Ghanim et al. (121) showed that a single oral dose of $100 \mathrm{mg}$ resveratrol plus $75 \mathrm{mg}$ of total polyphenols from a muscadine grape extract suppressed the mRNA and protein expression of inflammatory proteins induced by a high-fat, highcarbohydrate meal (\% of energy from carbohydrate:protein:fat; 41:17:42) in healthy subjects. However, we have not tested the acute effects of resveratrol. Moreover, it is 
also possible that the effects observed by Ghanim et al. (121) were due to the muscadine grape extract. In another study, sICAM-1 concentrations improved after 1year consumption of a grape supplement including $8 \mathrm{mg}$ resveratrol in patients who were statin-treated for primary prevention of cardiovascular disease (122). However, in our study, no effects on fasting or postprandial changes in plasma concentrations of inflammatory biomarkers were observed, which indicates that 4-wk $150 \mathrm{mg}$ daily transresveratrol consumption does not improve fasted or postprandial inflammatory markers in this population.

To conclude, no effects on vascular function as measured by FMD and PWV were found after 4-wk $150 \mathrm{mg} /$ day trans-resveratrol consumption. Furthermore, the lack of effect on postprandial glucose, insulin and TAG concentration, and postprandial inflammatory and endothelial function markers extends our earlier findings that $150 \mathrm{mg}$ trans-resveratrol daily for four weeks had no effect on these parameters in the fasted state. 


\section{CHAPTER 5}

Consuming a buttermilk drink containing lutein-enriched egg-yolk daily for 1 year increases plasma lutein but does not affect serum lipid or lipoprotein concentrations in adults with early signs of age-related macular degeneration

Sanne M. van der Made, Elton R. Kelly, Tos T.J.M. Berendschot, Aize Kijlstra, Dieter Lütjohann, Jogchum Plat 


\begin{abstract}
Dietary lutein intake is postulated to interfere with the development of age-related macular degeneration (AMD). Since egg-yolk derived lutein has a high bioavailability, long-term consumption of lutein-enriched eggs might be effective in preventing AMD development, but alternatively might increase cardiovascular disease risk. Here, we report the effect of 1-year daily consumption of a buttermilk drink containing one and a half lutein-rich egg-yolks on serum lipid, lipoprotein and plasma lutein concentrations. Additionally, subgroups that could potentially benefit most from the intervention were identified. Men and women who had early signs of AMD in at least one eye, but were otherwise healthy, participated in a one-year randomized, placebo-controlled parallel intervention trial. At the start of the study, 101 subjects were included; $N=52$ in the experimental group (Egg) and $\mathrm{N}=49$ in the control group (Con). Final analyses were performed with 45 subjects in the Egg group and 43 subjects in the Con group. As expected, the increase in plasma lutein concentrations in the Egg group was $83 \%$ greater than that in the Con group $(P<0.001)$. Changes in serum total-, HDL- and LDL cholesterol as well as the ratio of total:HDL cholesterol were not different between both groups. Interestingly, subjects classified as cholesterol absorber showed higher serum $\mathrm{HDL}$ cholesterol concentrations than subjects classified as cholesterol synthesizers or subjects with average campesterol:lathosterol ratios $(\mathrm{P}<0.05)$ at baseline. In addition, cholesterol absorbers showed a $229 \%$ greater increase in plasma lutein concentrations than subjects who were classified as having an average campesterol:lathosterol ratio ( $P$ $<0.05$ ) upon consumption of the lutein-enriched egg yolk drink. Moreover, the change in serum HDL cholesterol upon consumption was significantly different between these three groups $(P<0.05)$. We suggest that particularly cholesterol absorbers might benefit from the lutein-enriched buttermilk drink. This study was registered at clinicaltrials.gov as NCT00902408.
\end{abstract}




\section{Introduction}

Eggs provide several essential nutrients to the diet, such as high quality proteins, minerals, folate, and vitamins A, B12, D and K (123). In addition, one egg yolk contains on average $200 \mathrm{mg}$ of dietary cholesterol, which make eggs a main contributor of dietary cholesterol intake. The American Heart Association guidelines state that daily dietary cholesterol intake should not exceed 300 mg in healthy individuals (124), whereas for high-risk individuals, the recommended daily dietary cholesterol intake is even lower (< $200 \mathrm{mg}$ ) (125). Consuming elevated amounts of dietary cholesterol has been linked to an increase in the ratio of total to HDL cholesterol (126), suggesting an increased cardiovascular risk. Furthermore, a recent study suggests that especially subjects with elevated intestinal cholesterol absorption (so-called absorbers) are at risk for cardiovascular disease $(C V D)^{6}$ and not so much those with a high endogenous cholesterol synthesis (127). Interestingly, Herron et al. reported an increase in large LDL particles and a decrease in small LDL particles after increased dietary cholesterol intake from eggs, which is expected to result in a lower CVD risk (128). This illustrates that it is difficult to translate results into risk predictions. A possible reason for this could relate to the relatively short follow-up period of most interventions with typical durations ranging from 3 to 20 weeks. Therefore, long-term prospective follow up studies should be able to provide more consistent answers. Surprisingly, two recent long-term approaches reported completely opposite conclusions regarding the association between egg consumption and CVD risk $(129,130)$. This could relate to the assumption that it is difficult to relate CVD risk with a single nutrient or food product due to methodological constraints of such an approach (131). In addition, it is generally accepted that it is difficult to estimate food intake over longer periods back in time (132). Therefore, to overcome these issues, long-term well-controlled double blind intervention studies could potentially provide the best estimation of changes in cardiovascular risk.

Providing answers to the question whether frequent egg-intake should be discouraged or not is extremely relevant, especially because lutein and zeaxanthin from egg yolks is highly bioavailable (133), and these carotenoids are suggested to play a role in the prevention or treatment of specific diseases (134). Lutein from egg-yolks has a high bioavailability translating into increased plasma lutein concentrations (133). Within the circulation, lutein is primarily transported in HDL particles (135) to different target tissues such as the macular region of the retina (136). Consequently, a higher dietary lutein intake increases macular pigment optical density (MPOD). Both lutein and zeaxanthin are thought to play an important role in preserving the functional integrity of the retina, as these are major constituents of the macular pigment $(137,138)$. In other words, increased dietary consumption of these carotenoids might have a preventive effect on the risk for age-related macular degeneration (AMD). If true, this could be of 
great clinical and societal value since AMD is the most frequent cause of blindness in elderly in the Western world (139) and severely lowers quality of life (140).

Therefore, the primary objective of the present study was to assess the effects of 1year daily consumption of lutein-rich egg-yolks on plasma lutein concentrations, MPOD and visual function in subjects with early AMD (to be reported elsewhere) while the secondary objective - as discussed in this paper - was to evaluate the effects of longterm daily egg yolk consumption on serum lipids, and lipoproteins in order to estimate changes in cardiovascular risk. Moreover, since it is well known that there is a large inter-individual variation of dietary cholesterol absorption ranging from $20-70 \%$ (141) and additionally subjects can be classified as being cholesterol absorber or synthesizer $(142,143)$, we also tried to see whether we could identify more or less responsive subgroups. Another typical example of such a subgroup could be based on apoE genotype since the relation between apolipoprotein $\mathrm{E}$ genotype and cholesterol metabolism is well known $(144,145)$. Interestingly, apoE genotype also seems to be related to the risk for developing AMD (146), however, to the best of our knowledge, it is not known whether apoE genotype influences lutein metabolism.

\section{Subjects and Methods}

\section{Subjects}

The Medical Ethics Committee of Maastricht University Medical Centre approved the study protocol. The study was registered at clinicaltrials.gov as NCT00902408. One hundred and one subjects were recruited via advertisements in newspapers in the province of Limburg, The Netherlands. All participants gave their written informed consent before the screening procedure started. Eligible subjects were at least 50 years of age, did not receive treatment for diabetes or elevated serum cholesterol concentrations and had early signs of macular degeneration, as evidenced by drusen and / or retinal pigment epithelium alterations in at least one eye through fundus photography. Furthermore, intake of dietary supplements containing lutein or zeaxanthin was not allowed, and subjects allergic to eggs or egg-products were not included.

Twelve participants withdrew during the 1-year follow-up of the study. From these dropouts, six persons were part of the Egg group and six dropped out while being in the Con group. One subject started lutein supplementation during the intervention, two subjects started cholesterol lowering treatment via their general practitioner, four subjects suffered from digestive tract complaints after consuming the drinks (two in the Con and two in the Egg group) and four subjects withdrew because of various other 
physical complaints or personal reasons. As complaints were equally distributed over the Con and Egg group, we assumed that there was no relation with the experimental study product.

A total of 29 males and 60 females completed the study. One subject, who was part of the intervention group, was left out of analysis, because of outlying HDL cholesterol concentrations, as evidenced by a large Cook's distance. Final statistical analyses were performed on data from 88 subjects. The flow of participants throughout the entire study follow up is shown in figure 5.1.

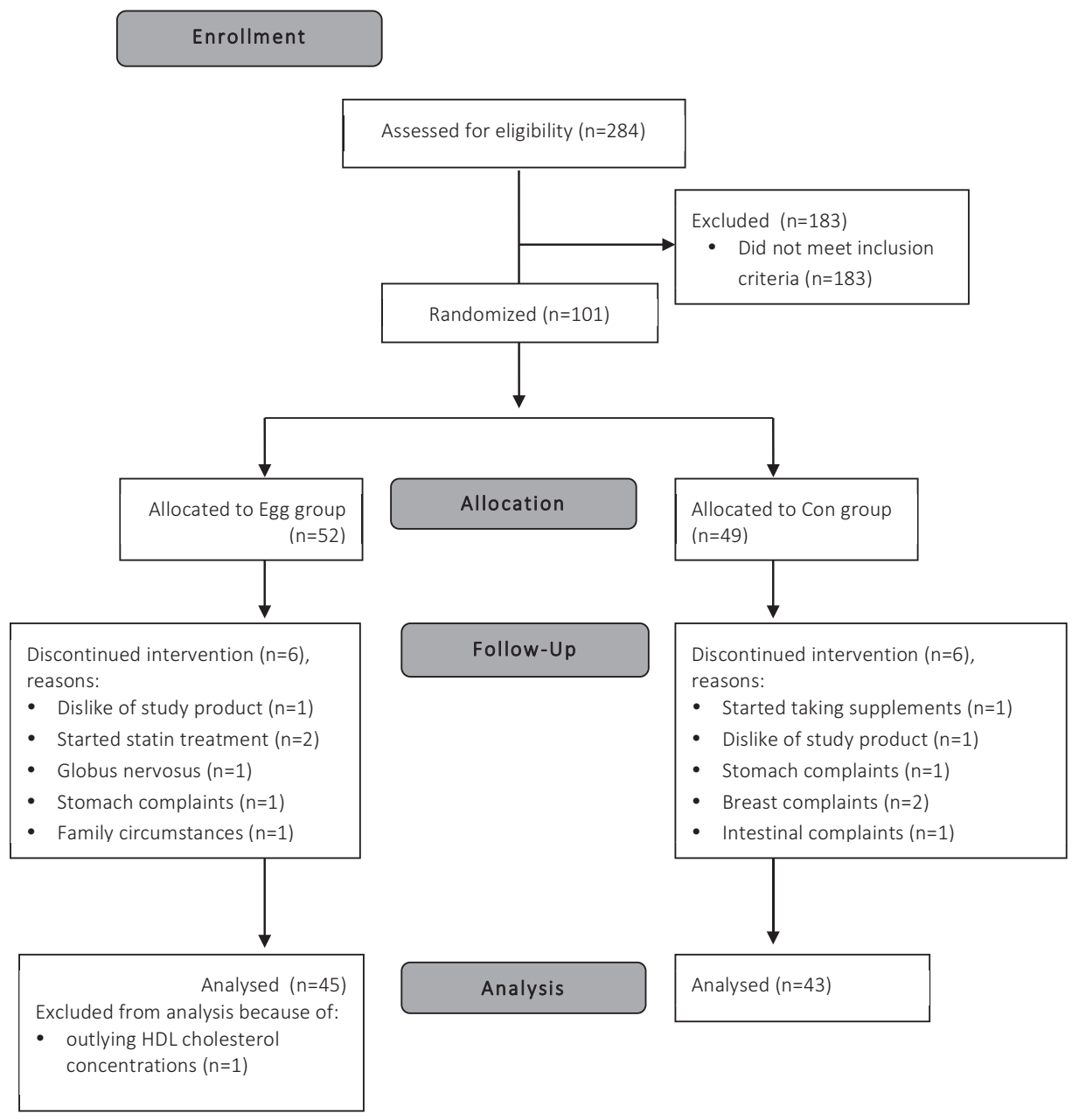

Figure 5.1 Participant flow chart. Con, control; Egg, experimental 


\section{Study design and intervention}

Subjects participated in a randomized, double blind, placebo-controlled one-year dietary intervention trial. Before the start of the study, subjects were randomly assigned to either the Egg or the Con group. Participants who were allocated to the Egg group were asked to consume a lutein-enriched egg-drink daily (provided by Newtricious R\&D, Oirlo, the Netherlands). For this, the egg-yolks used in the production of these drinks were enriched with lutein, through natural sources, via the feed of laying hens. The feed never exceeded the EU regulation concerning the maximal xanthophyll level of $80 \mathrm{ppm}$. One and a half egg-yolks were incorporated into an $80 \mathrm{~mL}$ buttermilk drink, i.e. the drink provided on average $1.4 \mathrm{mg}$ lutein and $323 \mathrm{mg}$ cholesterol per day for a total follow up period of one year. Subjects in the Con group received a comparable buttermilk drink, without the addition of egg yolks. Nutrient composition of both drinks is provided in Table 5.1.

\begin{tabular}{|c|c|c|}
\hline & Control drink & $\begin{array}{l}\text { Lutein-enriched } \\
\text { egg-yolk drink }\end{array}$ \\
\hline Energy (kcal) & 41 & 131 \\
\hline Fat (g) & 0.4 & 9.2 \\
\hline SFA (\%) & 67.0 & 32.5 \\
\hline TransFA (\%) & 3.1 & 0.3 \\
\hline MUFA (\%) & 26.2 & 49.5 \\
\hline PUFA (\%) & 3.7 & 17.7 \\
\hline Protein (g) & 2.7 & 6.2 \\
\hline Carbohydrate (g) & 6.6 & 5.8 \\
\hline Cholesterol (mg) & 2.0 & 323 \\
\hline Lutein (mg) & - & $1.4 \pm 0.2$ \\
\hline Zeaxanthin (mg) & - & $0.3 \pm 0.0$ \\
\hline
\end{tabular}

For blinding reasons, the color of the control drink was matched to the lutein-enriched egg drink by adding synthetic colorants E104 and E110. Participants received every two weeks a fresh delivery of products at home. At baseline and at the end of the intervention period, subjects were asked to fill out a validated food-frequency questionnaire (FFQ). These questionnaires were immediately checked by the research dietician, and used to calculate energy and nutrient intakes by the Dutch food composition table. Subjects were asked not to make large changes in their dietary pattern, level of physical exercise, use of alcohol and smoking habits during the one-year course of the study. 


\section{Blood sampling and analyses}

At the start (TO) and at the end of the intervention period (after 12 months, T12), blood was sampled after an overnight fast. Blood was sampled into serum separator tubes (Becton, Dickinson and Company, Franklin Lakes, NY, USA) for analysis of lipids, apolipoproteins and high-sensitivity C-reactive protein (hsCRP). These tubes were placed at room temperature for at least $30 \mathrm{~min}$ to allow clotting, followed by low-speed centrifugation at $1300 \mathrm{x}$ g for $15 \mathrm{~min}$ at $21^{\circ} \mathrm{C}$ to obtain serum. Fasting serum samples were enzymatically measured to determine serum total cholesterol by the CHOD-PAP method (Roche Diagnostics, Mannheim, Germany), HDL cholesterol (precipitation method; Roche Diagnostics, Mannheim, Germany) and triacylglycerol concentrations, with correction for free glycerol (GPO Trinder; Sigma-Aldrich Corp., St. Louis, MO, USA). ApoB100, ApoA-1 and hsCRP were analyzed by highly sensitive immunoturbidimetric assays (Horiba ABX, Montpellier, France). Serum LDL concentrations were calculated according to the Friedewald equation (2). Liver and kidney function parameters (creatinin, alkaline phosphatase (ALP), $\gamma$-glutamyl transpeptidase (GGT), aspartate aminotransferase (ASAT), alanine transaminase (ALAT) and total bilirubin) were measured in serum on a Beckman Coulter Synchron LX20 PRO Clinical System (Beckman Coulter Inc., Fullerton, CA, USA). Also a list of markers reflecting intestinal cholesterol absorption (sitosterol, campesterol and cholestanol) and endogenous cholesterol synthesis (lathosterol) were analyzed in serum by gas-liquid chromatography-mass spectroscopy (GC-MS) as described before (147).

Plasma was obtained by sampling blood into EDTA or sodium fluoride ( $\mathrm{NaF}$ ) tubes (Becton, Dickinson and Company, Franklin Lakes, NY, USA), followed by low-speed centrifugation at $1300 \mathrm{x}$ g for $15 \mathrm{~min}$ at $4^{\circ} \mathrm{C}$. Lutein and zeaxanthin concentrations were determined in EDTA plasma samples by high performance liquid chromatography (HPLC) as described (148). NaF plasma was used for analyzing circulating glucose concentrations (Horiba ABX, Montpellier, France). Also the non-esterified fatty acids (NEFA) (NEFA kit; WAKO Chemicals, Germany) concentrations were analyzed in fasted NaF plasma.

Immediately after centrifugation, both serum and plasma samples were snap-frozen into liquid nitrogen and stored at $-80^{\circ} \mathrm{C}$ until analysis. All indicated measurements were performed in the same assay to reduce inter-assay variation.

\section{ApoE genotyping}

DNA was collected from 87 participants that completed the study. DNA was isolated from the buffy coat layer of $10 \mathrm{~mL}$ blood collected in an EDTA-containing tube (Becton, Dickinson and Company, Franklin Lakes, NY, USA). The rs7412 and rs429358 single nucleotide polymorphisms (both coding for the APOE gene) were analyzed by Taqman Real Time PCR (Applied Biosystems). Subjects were divided into the apoE2 group (E2/2 and E2/3), E3 group (E3/3) or E4 group (E3/4 and E4/4). The E2/E4 genotype was 
excluded from grouping, since opposite effects of the E2 and E4 alleles on CVD risk have been described (149).

\section{Statistics}

Data are presented as mean \pm SD, unless otherwise indicated. Regarding serum lipid, lipoprotein and lutein concentrations, kidney and liver function parameters and FFQ outcomes, changes were calculated between the end of the intervention period and baseline. An independent student's T-test was performed to check for baseline differences and to test for differences in changes between the groups in the abovementioned parameters. Differences in gender distribution and smoking status over the Egg and Con groups were tested using the Pearson Chi-square test.

Analysis of subgroups based on apoE genotypes and ratio of campesterol:lathosterol (i.e. categorization into cholesterol synthesizers or absorbers) was performed using ANOVA to evaluate differences between subgroups concerning lipids, lipoproteins, and lutein concentrations at baseline and at the end of the intervention upon lutein-enriched eggdrink consumption. For this, BMI, gender and age were considered as potential confounders, because of their known interactions with cholesterol metabolism. As these parameters were equally distributed over the three apoE groups, we did not correct for them in this model. Thus, for evaluating differences between the ApoE subgroups at baseline and changes upon lutein-enriched egg-drink consumption 1-factor ANOVA was used. Gender and BMI were not equally distributed at baseline when the group was divided according to their campesterol:lathosterol ratio. Therefore, ANCOVA with gender and $\mathrm{BMI}$ as covariates was used to test for baseline differences and changes upon lutein-enriched egg-drink consumption in between the low-, mid- and high campesterol:lathosterol ratio groups. Post hoc comparisons with Bonferroni correction were carried out to compare apoE groups and low-, mid- and- high campesterol:lathosterol ratio groups.

Statistical power calculation was initially based on macular pigment optical density (MPOD), as this was the primary outcome of this project (to be reported elsewhere). The expected increase in MPOD in this trial was estimated at $12 \%$. Regarding an MPOD measuring error of $17 \%$, a significance level $(\alpha)$ of $5 \%$, a power of $90 \%$, and a $10 \%$ dropout rate, 48 subjects had to be included in the study. However, for the secondary outcome parameter, flow-mediated dilation (FMD), 50 subjects should be included in both intervention groups to detect a true difference in FMD of at least 1\%, regarding a standard deviation of FMD of $1.7 \%$, a drop-out rate of $10 \%$, a power of $80 \%$ and $\alpha=5 \%$. Therefore, a total of 101 subjects started the study.

Statistical analyses were carried out using IBM SPSS Statistics 21.0 for Macintosh OS $X$ package. Results were considered to be statistically significant if $P<0.05$. 


\section{Results}

Subject characteristics and dietary intake

\begin{tabular}{|c|c|c|c|c|c|c|}
\hline \multirow[b]{2}{*}{$\operatorname{Sex}(M / F)$} & \multicolumn{3}{|c|}{$\begin{array}{c}\text { Egg } \\
(\mathrm{N}=45)\end{array}$} & \multicolumn{3}{|c|}{$\begin{array}{c}\text { Con } \\
(\mathrm{N}=43)\end{array}$} \\
\hline & 15 & / & 30 & 14 & / & 29 \\
\hline Age (y) & 62 & \pm & 6 & 62 & \pm & 8 \\
\hline Smoking (yes/no) ${ }^{2}$ & 18 & / & 26 & 16 & / & 27 \\
\hline $\mathrm{BMI}\left(\mathrm{kg} / \mathrm{m}^{2}\right)$ & 26.9 & \pm & 3.6 & 26.3 & \pm & 3.7 \\
\hline Systolic blood pressure (mmHg) & 133 & \pm & 18 & 136 & \pm & 23 \\
\hline Diastolic blood pressure (mmHg) & 76 & \pm & 9 & 78 & \pm & 11 \\
\hline Plasma glucose (mmol/L) & 5.46 & \pm & 0.43 & 5.41 & \pm & 0.46 \\
\hline Serum hsCRP (mg/L) & 2.49 & \pm & 4.13 & 1.96 & \pm & 2.17 \\
\hline Plasma NEFA ( $\mu \mathrm{mol} / \mathrm{L})$ & 503 & \pm & 167 & 518 & \pm & 209 \\
\hline Serum creatinine $(\mu \mathrm{mol} / \mathrm{L})$ & 70.8 & \pm & 13.4 & 75.0 & \pm & 12.4 \\
\hline Serum ALP (IU/L) & 74.9 & \pm & 16.6 & 84.3 & \pm & $22.6^{*}$ \\
\hline Serum GGT (IU/L) & 28.2 & \pm & 16.7 & 24.7 & \pm & 12.6 \\
\hline Serum ASAT (IU/L) & 19.6 & \pm & 7.2 & 22.6 & \pm & 12.4 \\
\hline Serum ALAT (IU/L) & 21.6 & \pm & 6.6 & 23.9 & \pm & 12.6 \\
\hline Serum bilirubin ( $\mu \mathrm{mol} / \mathrm{L})$ & 15.0 & \pm & 4.5 & 16.6 & \pm & 7.1 \\
\hline \multicolumn{7}{|c|}{$\begin{array}{l}{ }^{1} \text { Values are means } \pm \text { SDs. }{ }^{*} \text { Different from Con, } P<0.05 \text {. ALAT; Alanine } \\
\text { transaminase, ALP; Alkaline phosphatase, ASAT; Aspartate transaminase, CRP; } \\
\text { high-sensitivity C-reactive protein, GGT; Gamma-glutamyltranspeptidase, NEFA; } \\
\text { non-esterified fatty acids. }\end{array}$} \\
\hline
\end{tabular}

At baseline, there were no differences between the Con and Egg group regarding age, gender, smoking status, BMI, systolic blood pressure, diastolic blood pressure, glucose, hsCRP and NEFA. Also, baseline liver function parameters were comparable between the groups, except for ALP concentrations, which were significantly greater $(P<0.05)$ in the Con group as compared to the Egg group (Table 5.2). This was due to relatively high ALP values in three subjects as compared to the total group, although these values were below the recommended upper limits for healthy liver function as established within the Maastricht University Medical Center+. 
Table 5.3 Total energy and nutrient intakes at baseline and change after a one-year intervention with the lutein-enriched egg-drink (Egg) or control beverage (Con) in adults with early signs of age-related macular degeneration1

\begin{tabular}{|c|c|c|c|c|c|c|c|c|c|c|c|c|}
\hline \multirow[b]{3}{*}{ Energy (MJ/d) } & \multicolumn{6}{|c|}{$\begin{array}{c}\text { Egg } \\
(N=43)\end{array}$} & \multicolumn{6}{|c|}{$\begin{array}{c}\text { Con } \\
(N=42)\end{array}$} \\
\hline & \multicolumn{3}{|c|}{ Baseline } & \multicolumn{3}{|c|}{ Change } & \multicolumn{3}{|c|}{ Baseline } & \multicolumn{3}{|c|}{ Change } \\
\hline & 9.7 & \pm & 2.2 & -0.6 & \pm & $2.3^{\#}$ & 9.3 & \pm & 3.3 & -0.6 & \pm & 2.7 \\
\hline Protein (En\%) & 18 & \pm & 5.9 & -0.2 & \pm & 5.0 & 18 & \pm & 3.8 & 0.6 & \pm & 3.8 \\
\hline Fat Total (En\%) & 39 & \pm & 7.2 & 2.6 & \pm & 6.4 & 40 & \pm & 7.9 & 1.0 & \pm & 9.8 \\
\hline SAFA (En\%) & 13 & \pm & 3.1 & 0.9 & \pm & 3.0 & 14 & \pm & 3.0 & -0.2 & \pm & 3.6 \\
\hline MUFA (En\%) & 14 & \pm & 4.0 & 0.5 & \pm & $3.5^{\#}$ & 14 & \pm & 4.0 & 1.1 & \pm & 6.0 \\
\hline $\begin{array}{l}\text { Oleic Acid } \\
\text { (En\%) }\end{array}$ & 12 & \pm & 4.0 & -1.2 & \pm & $3.6^{* \#}$ & 12 & \pm & 3.9 & 1.2 & \pm & 5.6 \\
\hline TransFA (En\%) & 1.1 & \pm & 0.5 & 0.1 & \pm & 0.4 & 1.2 & \pm & 0.6 & -0.1 & \pm & 0.6 \\
\hline PUFA (En\%) & 7.9 & \pm & 3.2 & 1.4 & \pm & 3.3 & 8.2 & \pm & 4.0 & 0.3 & \pm & 4.4 \\
\hline $\begin{array}{l}\text { Carbohydrates } \\
\text { (En\%) }\end{array}$ & 41 & \pm & 9.5 & -0.8 & \pm & 9.0 & 43 & \pm & 9.0 & -0.2 & \pm & 12 \\
\hline Alcohol (En\%) & 4.2 & \pm & 3.8 & -0.7 & \pm & $2.0^{\#}$ & 3.0 & \pm & 3.3 & -0.3 & \pm & 2.4 \\
\hline Fiber $(g / d)$ & 24 & \pm & 8.5 & -1.4 & \pm & 7.9 & 26 & \pm & 11 & -2.5 & \pm & 11 \\
\hline $\begin{array}{l}\text { Cholesterol } \\
(\mathrm{mg} / \mathrm{d})\end{array}$ & $\begin{array}{r}24 \\
5\end{array}$ & \pm & 99 & 296 & \pm & $106^{* \#}$ & 217 & \pm & 103 & -12 & \pm & 83 \\
\hline $\operatorname{EPA}(\mathrm{g} / \mathrm{d})$ & 0.1 & \pm & 0.1 & -0.1 & \pm & $0.2^{\#}$ & 0.2 & \pm & 0.2 & -0.0 & \pm & 0.2 \\
\hline $\mathrm{DHA}(\mathrm{g} / \mathrm{d})$ & 0.2 & \pm & 0.2 & -0.1 & \pm & $-0.3^{\#}$ & 0.3 & \pm & 0.3 & -0.0 & \pm & 0.3 \\
\hline
\end{tabular}

${ }^{1}$ Values are means \pm SDs. ${ }^{*}$ Change differed from Con, $\mathrm{P}<0.05$

"Change within the group differed, $P<0.05$.

EPA; eicosapentaenoic acid, DHA; docosahexaenoic acid, MUFA; monounsaturated fatty acid, OA; oleic acid, PUFA; polyunsaturated fatty acid, SAFA; saturated fatty acid.

Total energy and nutrient intakes were calculated from the FFQ at baseline and after twelve months.

Subjects were asked to recall their dietary habits from the previous month. We were unable to obtain

FFQ results from two subjects in the Egg group and from one subject in the Con group.

No statistical significant differences were found at baseline.

Statistical significant changes within a group were assessed by a Student's Paired T-test.

At baseline, total energy intake, proportion of energy from proteins, fats, and carbohydrates, alcohol, fiber and cholesterol intake was comparable between the two groups. During the intervention period, dietary cholesterol intake was greater in the Egg group than in the Con group $(P<0.001)$, which was anticipated since this was the targeted intervention. In addition, oleic acid intake was significantly lower in the Egg group $(P<0.05$, Table 5.3). 


\section{Plasma lutein and zeaxanthin}

Baseline plasma lutein concentrations were comparable between the Egg and Con group and also between men and women. Increases in plasma lutein and zeaxanthin concentrations were $83 \%$ and $110 \%$ greater in the Egg group than in the Con group $(P<$ 0.001 and $P<0.01$ respectively), which reflected good compliance. Gender specific analysis revealed that these changes were evident in both men and women, however, increases in both lutein and zeaxanthin were more pronounced in men (127\%; $\mathrm{P}<0.01$ and $110 \% ; \mathrm{P}<0.05)$ than in women $(77 \% ; \mathrm{P}<0.01$ and $45 \% ; \mathrm{P}<0.05$ respectively). In the Egg group, no significant differences at baseline and in change during the intervention between men and women were found.

Since lutein and zeaxanthin are transported by lipoproteins in the circulation, we also calculated the cholesterol standardized plasma lutein concentrations to correct for changes in carrier capacity. After standardization of lutein for serum total cholesterol, an $83 \%$ greater increase in cholesterol-standardized lutein $(P<0.001)$ and a $50 \%$ greater increase in cholesterol-standardized zeaxanthin $(P<0.001$, Table 5.4) was observed in the Egg group than in the Con group. This suggests that the conclusion is similar to the conclusion for non-standardized lutein concentrations and therefore changes in lutein and zeaxanthin were not exclusively determined by changes in serum lipid and lipoprotein concentrations.

\section{Serum lipids and lipoproteins}

Baseline lipid and lipoprotein concentrations were comparable between the egg-drink (Egg) and control group (Con). No differences were found between both groups regarding the change in serum total, $\mathrm{LDL}$ and $\mathrm{HDL}$ cholesterol, total:HDL cholesterol ratio, apoB100, apoAI, TG and NEFAs (Table 5.4). Also, no differences were found in changes in serum lipids and lipoproteins throughout the intervention between men and women (data not shown). Also, the expected increases in total-, HDL- and LDL cholesterol, and total:HDL cholesterol ratio were calculated according to the prediction formula based on the meta-analysis by Weggemans et al. (126). Interestingly, the measured changes in total-, HDL- and LDL cholesterol after 12 months were fully in line with these predictions, whereas the change in total:HDL cholesterol ratio was lower than expected (Table 5.5). As shown in Figure 5.2, individual responses in total cholesterol concentration during the one-year course of lutein-enriched egg-drink consumption did not show remarkable differences between subjects. 
Table 5.4 Baseline values and changes during a one-year intervention with the lutein-enriched egg-drink (Egg) or control beverage (Con) in lipids, (apo)lipoproteins, markers for cholesterol metabolism and carotenoids in adults with early signs of age-related macular degeneration ${ }^{1}$

\begin{tabular}{|c|c|c|c|c|c|c|c|c|c|c|c|c|}
\hline \multirow[b]{3}{*}{$\begin{array}{l}\text { Serum total cholesterol } \\
(\mathrm{mmol} / \mathrm{L})\end{array}$} & \multicolumn{6}{|c|}{$\begin{array}{c}\text { Egg } \\
(N=45)\end{array}$} & \multicolumn{6}{|c|}{$\begin{array}{c}\text { Con } \\
(N=43)\end{array}$} \\
\hline & \multicolumn{3}{|c|}{ Baseline } & \multicolumn{3}{|c|}{ Change } & \multicolumn{3}{|c|}{ Baseline } & \multicolumn{3}{|c|}{ Change } \\
\hline & 6.01 & \pm & 1.14 & 0.30 & \pm & 0.69 & 6.04 & \pm & 1.15 & 0.13 & \pm & 0.54 \\
\hline $\begin{array}{l}\text { Serum LDL cholesterol } \\
\qquad(\mathrm{mmol} / \mathrm{L})\end{array}$ & 3.82 & \pm & 1.06 & 0.24 & \pm & 0.62 & 3.87 & \pm & 0.98 & 0.09 & \pm & 0.45 \\
\hline $\begin{array}{l}\text { Serum HDL cholesterol } \\
\qquad(\mathrm{mmol} / \mathrm{L})\end{array}$ & 1.65 & \pm & 0.41 & 0.04 & \pm & 0.18 & 1.67 & \pm & 0.39 & 0.01 & \pm & 0.18 \\
\hline $\begin{array}{l}\text { total:HDL cholesterol } \\
\text { ratio }\end{array}$ & 3.87 & \pm & 1.33 & 0.08 & \pm & 0.59 & 3.75 & \pm & 0.96 & 0.14 & \pm & 0.43 \\
\hline $\begin{array}{l}\text { Serum apoB100 } \\
\qquad(\mathrm{g} / \mathrm{L})\end{array}$ & 0.99 & \pm & 0.24 & 0.04 & \pm & 0.11 & 0.99 & \pm & 0.22 & 0.02 & \pm & 0.09 \\
\hline $\begin{array}{l}\text { Serum apoAl } \\
\qquad(\mathrm{g} / \mathrm{L})\end{array}$ & 1.38 & \pm & 0.21 & 0.02 & \pm & 0.11 & 1.38 & \pm & 0.21 & 0.01 & \pm & 0.16 \\
\hline $\begin{array}{l}\text { Serum TG } \\
\qquad(\mathrm{mmol} / \mathrm{L})\end{array}$ & 1.18 & \pm & 0.63 & 0.06 & \pm & 0.35 & 1.09 & \pm & 0.45 & 0.08 & \pm & 0.49 \\
\hline $\begin{array}{l}\text { Plasma NEFA } \\
(\mu \mathrm{mol} / \mathrm{L})\end{array}$ & 503 & \pm & 167 & -8 & \pm & 154 & 518 & \pm & 209 & -31 & \pm & 163 \\
\hline $\begin{array}{l}\text { Cholestanol/cholesterol } \\
\qquad\left(\mu \mathrm{mol} / \mathrm{mmol} \times 10^{-2}\right)\end{array}$ & 214 & \pm & 36 & -1 & \pm & 18 & 219 & \pm & 32 & 0 & \pm & 17 \\
\hline $\begin{array}{l}\text { Lathosterol/cholesterol } \\
\qquad\left(\mu \mathrm{mol} / \mathrm{mmol} \times 10^{-2}\right)\end{array}$ & 114 & \pm & 42 & 6 & \pm & 25 & 106 & \pm & 44 & 5 & \pm & 25 \\
\hline $\begin{array}{l}\text { Campesterol/cholester } \\
\text { ol }(\mu \mathrm{mol} / \mathrm{mmol} \times 10 \\
\left.{ }^{2}\right)\end{array}$ & 197 & \pm & 86 & 5 & \pm & 56 & 246 & \pm & 93 & -7 & \pm & 45 \\
\hline $\begin{array}{r}\text { Sitosterol/cholesterol } \\
\left(\mu \mathrm{mol} / \mathrm{mmol} \times 10^{-2}\right)\end{array}$ & 140 & \pm & 53 & 4 & \pm & 33 & 171 & \pm & 68 & -5 & \pm & 34 \\
\hline $\begin{array}{l}\text { Plasma lutein } \\
(\mathrm{ng} / \mathrm{mL})\end{array}$ & 210 & \pm & 150 & 189 & \pm & $154^{*}$ & 201 & \pm & 119 & 14.0 & \pm & 172 \\
\hline $\begin{array}{l}\text { Plasma zeaxanthin } \\
(\mathrm{ng} / \mathrm{mL})\end{array}$ & 67 & \pm & 46 & 35 & \pm & $49^{*}$ & 60 & \pm & 34 & -1.3 & \pm & 51 \\
\hline $\begin{array}{l}\text { Lutein/cholesterol } \\
\qquad(\mu \mathrm{mol} / \mathrm{mmol})\end{array}$ & 0.06 & \pm & 0.04 & 0.05 & \pm & $0.04^{*}$ & 0.06 & \pm & 0.03 & 0.00 & \pm & 0.04 \\
\hline $\begin{array}{l}\text { Zeaxanthin/cholesterol } \\
\text { ( } \mu \mathrm{mol} / \mathrm{mmol})\end{array}$ & 0.02 & \pm & 0.01 & 0.01 & \pm & $0.01^{*}$ & 0.02 & \pm & 0.01 & 0.00 & \pm & 0.01 \\
\hline
\end{tabular}

${ }^{1}$ Values are means \pm SDs. ${ }^{*}$ Change differed from Con, $\mathrm{P}<0.001$ 
Markers for cholesterol metabolism and classification of responders and non-responders

Cholesterol-standardized cholestanol, lathosterol, campesterol and sitosterol serum concentrations are shown in Table 5.4. No significant differences in change in these parameters were observed throughout the intervention.

Next, the serum campesterol:lathosterol ratio was calculated in order to classify subjects as being cholesterol synthesizers (low ratio) or cholesterol absorbers (high ratio). Subjects were classified in tertiles having a low $(\leq 1.44)$, middle $(1.45-1.66)$, or high ( $\geq 1.67)$ campesterol:lathosterol ratio. Subjects in the average $(P<0.05)$ or high group $(P<0.01)$ had a significant higher baseline HDL cholesterol concentration as compared to subjects with a low campesterol:lathosterol ratio, whereas other markers were not significantly different between these groups (Table 5.6). Baseline HDL cholesterol was $18 \%$ higher in the mid-ratio $(P<0.05)$ and $21 \%$ higher in the high-ratio group $(P<0.05)$ as compared to the low-ratio group. This suggests that subjects classified as being cholesterol absorbers might have a higher serum HDL cholesterol concentration as compared to those classified as synthesizers. Interestingly, also the change in HDL cholesterol upon lutein-enriched egg-drink consumption was significantly different between the three campesterol:lathosterol ratio groups $(P<0.05)$. Although this significant change was found, post-hoc comparison could not reveal whether this could be attributed to the low-, middle- or high-campesterol:lathosterol ratio group. Only a trend towards a significant greater increase in HDL cholesterol in the cholesterol absorbers compared to the middle campesterol:lathosterol ratio group was observed ( $P$ $=0.07)$, which might be caused by a lack of statistical power, because of the small subgroups. In other words, the tertile with the highest baseline HDL cholesterol concentrations also showed the highest increase in HDL cholesterol upon egg-yolk consumption. Interestingly, plasma lutein concentration in the high-ratio group was $229 \%$ greater than in the mid-ratio group $(P<0.05)$, whereas plasma lutein concentration was $172 \%$ greater than in the low-ratio group ( $P=0.08$; Table 5.6).

Table 5.5 Observed changes during a one-year intervention with a lutein-enriched egg-drink ( $N=45)$ compared to placebo $(N=43)$ and expected changes based on prediction equations (126) in total-, HDL- and LDL cholesterol concentrations and total:HDL cholesterol ratio ${ }^{1}$

\begin{tabular}{lcc}
\hline & $\begin{array}{c}\text { Expected } \\
\text { change }\end{array}$ & $\begin{array}{c}\text { Observed relative change } \\
\text { after } 12 \text { months }\end{array}$ \\
\hline Serum total cholesterol, $\mathrm{mmol} / \mathrm{L}$ & $0.18 \pm 0.02$ & $0.18 \pm 0.13$ \\
Serum HDL cholesterol, $\mathrm{mmol} / \mathrm{L}$ & $0.03 \pm 0.00$ & $0.03 \pm 0.04$ \\
Serum LDL cholesterol, $\mathrm{mmol} / \mathrm{L}$ & $0.16 \pm 0.01$ & $0.15 \pm 0.12$ \\
total:HDL cholesterol ratio & $0.06 \pm 0.02$ & $-0.06 \pm 0.11$ \\
\hline${ }^{1}$ Values are means \pm SEEs for expected increase and means \pm SEs for observed \\
change after 12 months
\end{tabular}


A

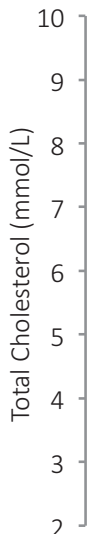

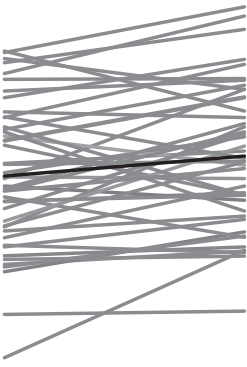

Month 0
B

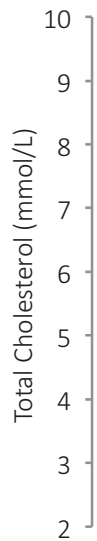

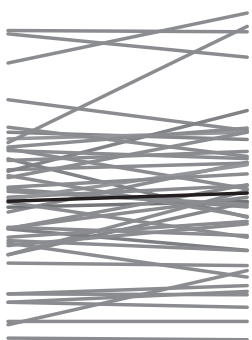

Month 0
Month 12

Figure 5.2 Individual total cholesterol concentrations (dark grey lines) at baseline and after one year of consuming the lutein-enriched egg-drinks in adults with early signs of macular degeneration enrolled in the Egg group (panel A) and the Con group (panel B). The black lines represent the group averages at the start and after 12 months. 


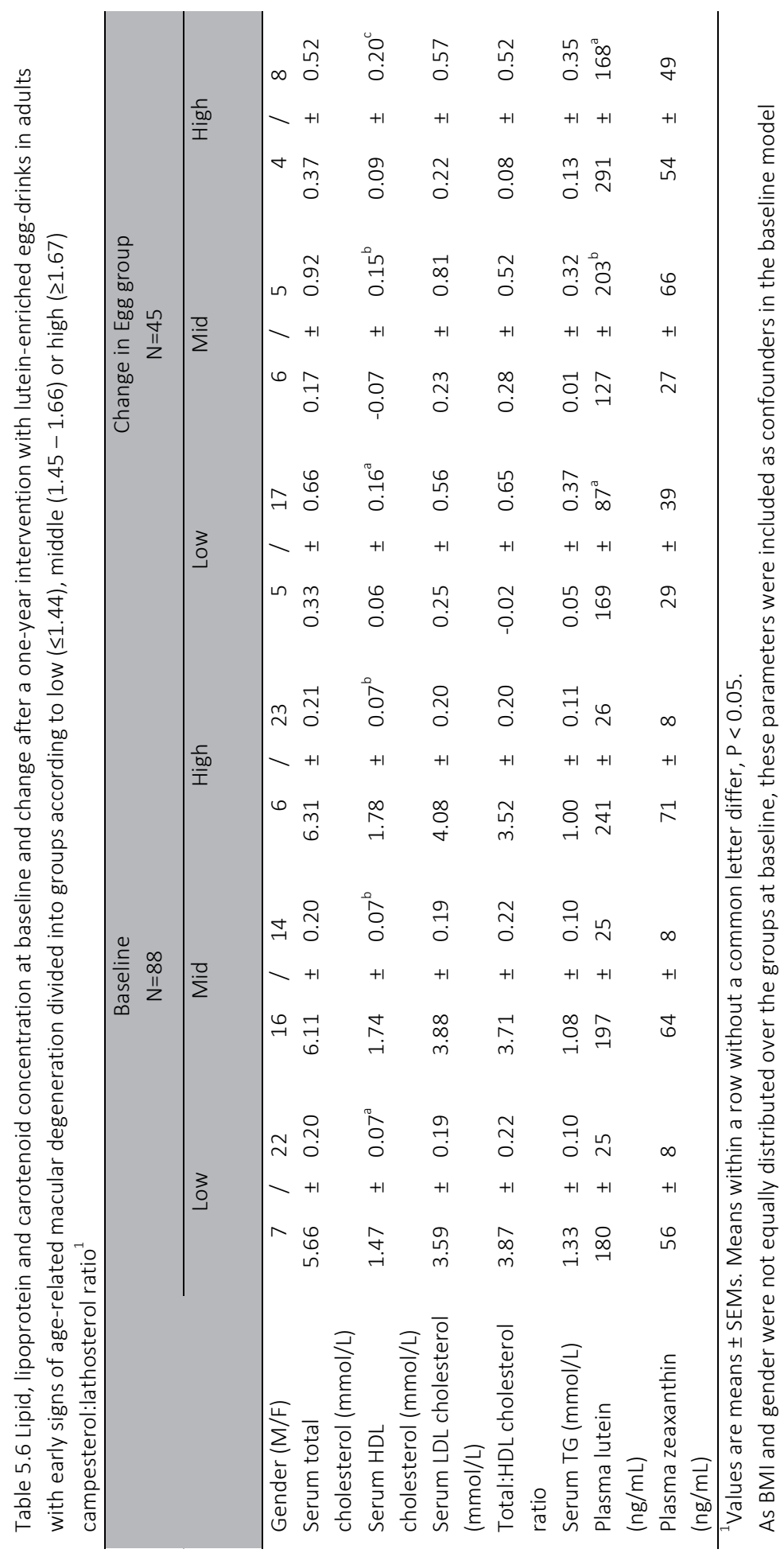


Table 5.7 Frequency distribution of the different genotypes in loci encoding apoE in adults with early signs of age-related macular degeneration

\begin{tabular}{llll}
\hline & ApoE2 & ApoE3 & ApoE4 \\
\hline Comp (n, \%) & $15,18.1$ & $50,60.2$ & $18,21.7$ \\
Egg $(n, \%)$ & $5,12.2$ & $26,63.4$ & $10,24.4$ \\
Con $(n, \%)$ & $10,23.8$ & $24,57.1$ & $8,19.0$ \\
\hline
\end{tabular}

Mean \pm SD

*Significantly different from apoE3 group $(\mathrm{P}<0.05)$.

Egg; egg-drink group, Con; control group, Comp;

complete group

ApoE2: E2/2 + E2/3; ApoE3: E3/3; ApoE4: E3/4 + E4/4

The effect of apoE genotype on circulating lipid lipoprotein, lutein and zeaxanthin concentrations

A total of 83 subjects were included in analyzing the effect of apoE genotype on fasting lipids and carotenoids; DNA was collected from 87 participants, of which two subjects had the apoE2/4 genotype and were excluded from further analysis due to impossible classification in the apoE2, E3 or E4 groups (149). We were unable to determine apoE genotype from one of the subjects, and another subject was left out of analysis because of outlying $\mathrm{HDL}$ cholesterol concentrations. Frequency distribution of the different genotypes in loci encoding apoE is shown in Table 5.7 and was in line with that in the general population (149). Baseline serum total- and LDL cholesterol concentrations were respectively $23 \%(P<0.05)$ and $22 \%(P<0.05)$ lower in subjects with the apoE2 genotype as compared to the apoE3 group (Table 5.8). Figure 5.3 shows the individual variation in baseline plasma lutein concentrations as well as the individual changes in plasma lutein concentrations after one year follow up classified according to the different apoE groups. There were no significant differences in the mean changes in lipid, lipoprotein or carotenoid concentrations between the three apoE genotypes during the lutein-enriched egg-drink consumption (Table 5.8). Results for differences in cholesterol standardized lutein and zeaxanthin concentrations between groups based on apoE genotype were essentially the same as those of absolute concentrations (data not shown). 


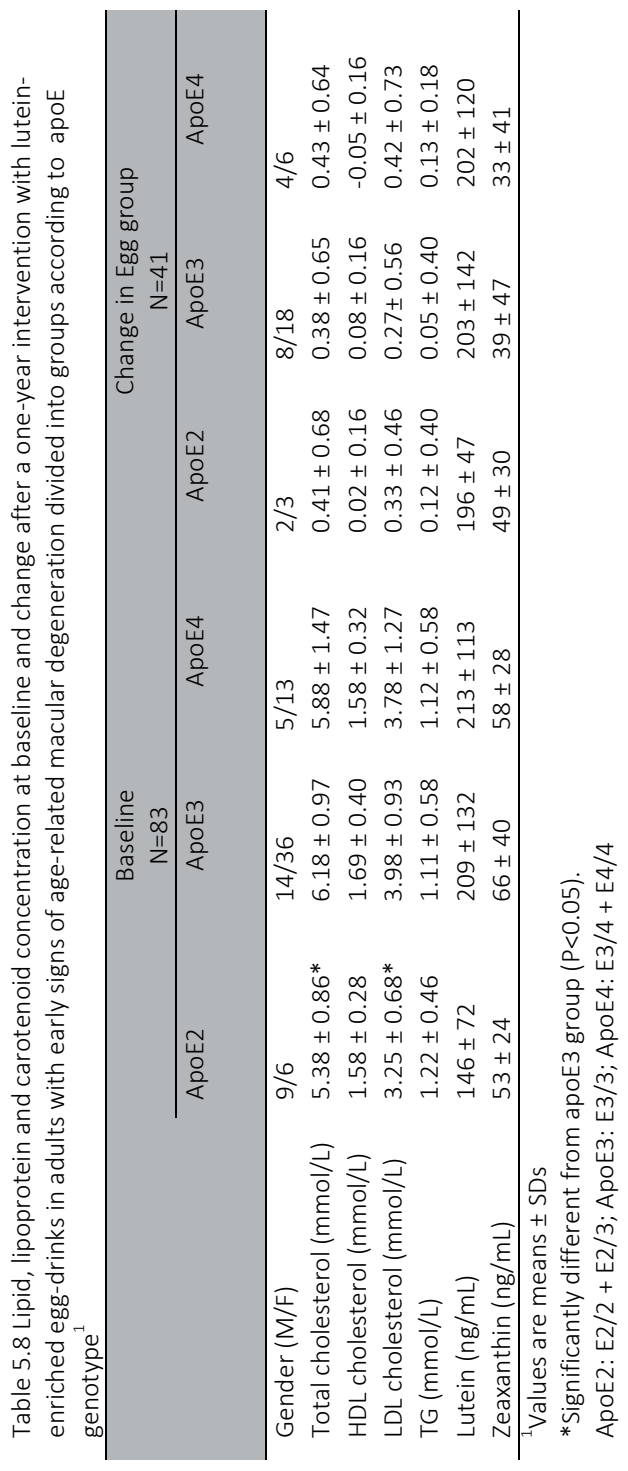


A

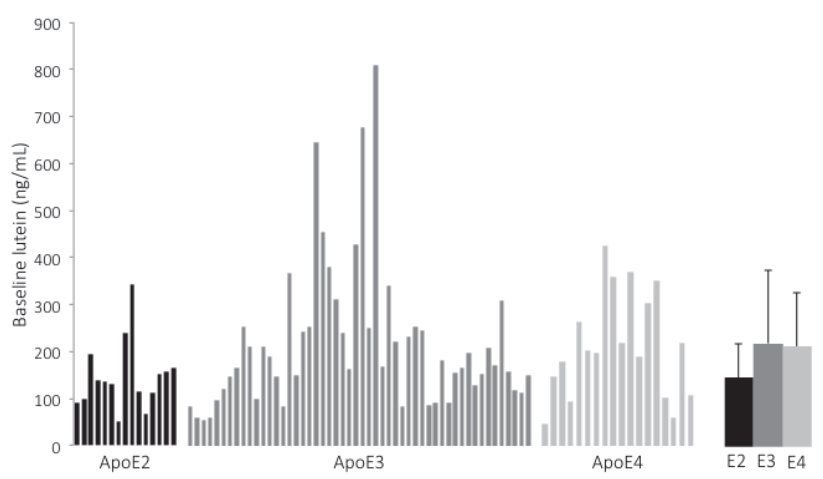

B

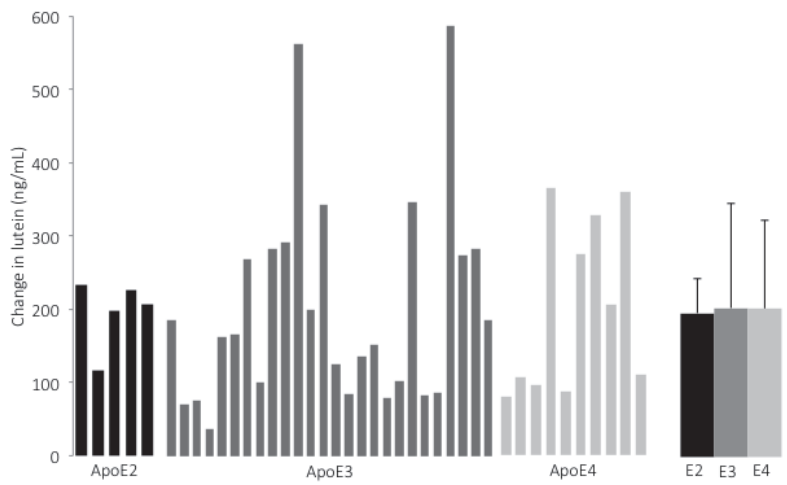

C

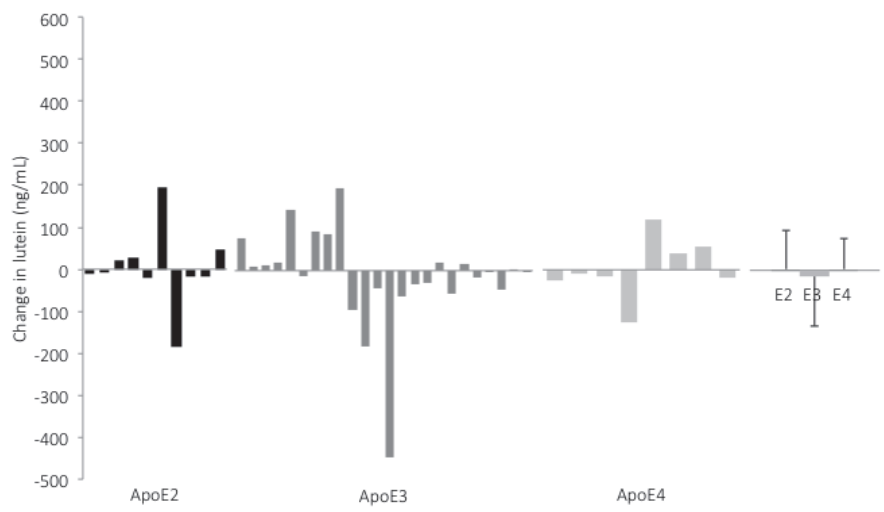

Figure 5.3 Individual baseline lutein concentrations (A), changes in lutein concentrations in the Egg group (B) and changes in lutein concentrations in the Con group (C) according to apoE genotype.

ApoE2: E2/2 +E2/3; ApoE3: E3/3; ApoE4: E3/4 + E4/4 


\section{Markers for kidney- and liver function}

Changes in kidney- and liver function parameters were monitored during the intervention to be able to observe any side effects. Apart from a greater increase in GGT in the Con group than in the Egg group during the intervention $(P<0.05)$, no significant differences were found (data not shown). Most important, during the one-year follow up period all liver and kidney function parameters remained below the reference values as established within the Maastricht University Medical Center+.

\section{Discussion}

This study shows that the consumption of a lutein-enriched egg yolk for a time period of one year leads to a significant increase in plasma lutein concentration. Both epidemiological as well as intervention studies indicate that a higher lutein intake may delay AMD development $(150,151)$. Since eggs are a known source of dietary lutein we started a study to investigate the effects of lutein-enriched eggs on visual parameters (to be reported elsewhere) as well as the possible side effects. An egg-containing beverage is well suited for a placebo-controlled trial since the control beverage can be adjusted for taste and colour properties. Moreover, the bioavailability of lutein from eggs is known to be higher than from lutein supplements (133).

During this one-year follow-up, serum total- and LDL cholesterol increased in line with the available prediction equations (126), however changes did not reach statistical significance. Based on different prediction models, the observed changes in serum lipids and lipoproteins imply a modest risk elevation. Whether the assumed beneficial effects on AMD outweigh the changed CVD risk deserves further analysis. Most important, we were able to identify a subpopulation of so-called cholesterol absorbers that presented themselves with significantly higher baseline HDL cholesterol concentrations, and showed a more pronounced increased plasma lutein concentration as compared to subjects categorized as cholesterol synthesizers based on their campesterol:lathosterol ratio. In theory, this is regarded the ideal target population for a lutein enriched egg-yolk containing dairy drink approach as preventive strategy against AMD development.

The observed increase in plasma lutein concentrations during the one-year daily lutein enriched egg-yolk containing drink consumption was in line with earlier short-term observations $(152,153)$. This suggests that there appears a plateau in serum lutein concentrations after interventions with a shorter duration of 4-12 weeks (152), obviously depending on the quantity and form of lutein supplementation. Although our lutein enriched dairy drink contained less lutein (1.5 mg per serving) in comparison with conventional supplements (10-20 mg), the lipid matrix in which the lutein was provided has been shown to increase its bioavailability (154). The $83 \%$ increase in plasma lutein concentrations resulted in an average plasma lutein concentration of $399 \mathrm{ng} / \mathrm{mL}$. Earlier, 
Berendschot et al. (2000) showed a stabilized serum lutein concentration around 340 $\mathrm{ng} / \mathrm{mL}$ after four weeks of $10 \mathrm{mg}$ lutein supplementation in a comparable population (152). From these results, we can conclude that supplementation of lutein via luteinenriched egg yolks incorporated in a buttermilk drink is at least as efficient in increasing plasma lutein concentrations as supplementation via supplements, since much lower quantities of lutein are needed to reach comparable plasma lutein concentrations. Whether this translates into increased MPOD values and slows down the regression of AMD needs further study (manuscript in preparation).

An important issue related to the use of an egg-yolk matrix that needs to be addressed is how daily consumption of 1.5 egg-yolks translates into changes in cardiovascular risk. Although the observed increases in total- and LDL cholesterol were not significant, they were fully in line with the expectations according prediction equations by Weggemans et al. (126). These predictions are based on studies with a maximum intervention duration of 20 weeks. Based on our data, we can conclude that cholesterol metabolism has clearly reached a new steady state already after these 20 weeks since changes in serum cholesterol concentrations as observed after 1 year follow up are comparable to the prediction outcome (Table 5.5). The question what the observed changes in serum lipids and lipoproteins mean in terms of cardiovascular risk prediction is a highly relevant question given the on-going discussion regarding the need for restriction of egg consumption in the dietary guidelines. The outcome of future CVD risk prediction depends on whether changes in LDL cholesterol or changes in the total:HDL cholesterol ratio are used. A $1 \mathrm{mmol} / \mathrm{L}$ decrease in $\mathrm{LDL}$ cholesterol concentrations is associated with a $20 \%$ lowering in risk for coronary events such as myocardial infarction and ischemic stroke $(155,156)$. If one would translate this curvelinear relation from the included pharma trials to the results from our dietary intervention study, an increase in LDL cholesterol of $0.14 \mathrm{mmol} / \mathrm{L}$, if this were significant, would assume a modest increase in risk for coronary events of $2.8 \%$. However, the observed decrease in the total:HDL cholesterol ratio is related to a $0.7 \%$ decrease in risk for future CHD (157). Moreover, a strong correlation between the change in apoB100 and LDL cholesterol concentrations $(r=0.822, P<0.001)$ was found in the present study. This is in line with studies that showed a parallel between change in apoB100 concentration and LDL cholesterol concentrations (158-161), and implies that there is no change in the amount of cholesterol per LDL particle. In other words, there is apparently no change in LDL particle size or density. Earlier studies suggested that egg consumption shifts the LDL fraction towards the less atherogenic LDL-1 subclass (162). We could, however, not confirm this suggestion after consuming lutein-enriched egg-drinks for one year. Next, we also explored the effect of consuming the lutein-enriched egg drink on markers for cholesterol metabolism, i.e. markers for endogenous cholesterol synthesis and fractional intestinal cholesterol absorption. Earlier short-term studies did not show 
an effect on these markers after consumption of 1 additional egg yolk a day for 12 weeks (163). We here confirm our prior observation by showing that these markers are not affected after one-year additional daily intake of on average $323 \mathrm{mg}$ dietary cholesterol provided through 1.5 egg yolks. However, one should consider that using lathosterol as marker for endogenous synthesis in the situation of egg-consumption may not be ideal since egg-yolks have been suggested as a lathosterol rich source (164).

It is generally accepted that there is a large inter-individual variation in dietary cholesterol absorption ranging between 20-70\% (141). In addition, subjects can be classified as being cholesterol absorber or synthesizer (142). An individual with a high campesterol:lathosterol ratio identifies a cholesterol absorber, whereas a low ratio classifies a so-called synthesizer (143). Based on this classification we attempted to see whether we could identify more or less responsive subgroups, both in lutein as well as regarding lipid and lipoprotein concentrations. We here show that the so-called cholesterol-absorbers had significantly higher baseline HDL cholesterol concentrations. Next to this, these subjects also showed a higher increase in serum HDL cholesterol and significantly higher serum lutein concentrations after one-year egg-yolk consumption as compared to cholesterol synthesizers and subjects in the mid campesterol:lathosterol ratio group. From this, we conclude that cholesterol absorbers might benefit most from the lutein-enriched egg-yolk drink, not only because serum lutein increased most pronounced, but also additionally serum HDL cholesterol concentrations increased significantly. Importantly, no significant differences in change in LDL cholesterol and of the total:HDL cholesterol ratio between the three subgroups were observed. It is important to realize that the change in LDL cholesterol in the absorbers, if this change were significant, corresponds to a $4.4 \%$ increase in risk for future CVD, whereas the increase in total:HDL cholesterol ratio relates to a $1.0 \%$ increase in this risk, which are both slightly higher than calculated in the entire population. Although this risk for cardiovascular events cannot be outweighed, we need to point out that the egg-yolk based buttermilk drink provided in this intervention study was enriched with highly bioavailable lutein, which might be beneficial for (cardiovascular) health (165).

A second typical example of such a subgroup could be based on apoE genotype since the relation between apolipoprotein $\mathrm{E}$ and cholesterol metabolism is well known $(149,166)$. Moreover, a pooled analysis of data from studies evaluating the relation between apoE genotype and AMD provided evidence for a protective role of the $\varepsilon 4$ haplotype in AMD and a significant increased risk associated with the $\varepsilon 2 \varepsilon 2$ diplotype for late AMD. Next to this, it is well known that the $\varepsilon 4$ isoform predisposes for higher intestinal cholesterol absorption efficiency, while the $\varepsilon 2$ isoform is associated with a lower fractional intestinal cholesterol absorption (146). This would be an ideal condition for the development of personalized nutrition strategies using the lutein enriched eggyolk drink, since the apoE2 group is characterized by low plasma lutein concentrations and at the same time they are not expected to respond as strongly with an increase in 
total cholesterol or LDL cholesterol concentrations. However, possibly resulting from the relatively small sample size in this subanalysis, we conclude that, in contrast to the campesterol:lathosterol ratio, apoE genotype cannot be used as subgroup classification to identify lutein responsive subgroups, based on the present results. Further studies using a larger sample size are needed to confirm this hypothesis.

In conclusion, consuming lutein-enriched egg yolks incorporated into a buttermilk drink daily for a time period of one year resulted in a significantly increased plasma lutein concentration, without significantly increasing lipid or (apo)lipoprotein concentrations. A subgroup that potentially benefits most from the lutein-enriched eggdrink approach is subjects characterized as cholesterol-absorbers. They showed a larger increase in HDL cholesterol during intervention, notwithstanding their already higher baseline HDL cholesterol concentrations, and at the same time responded with a more pronounced increase in plasma lutein concentration.

\section{Acknowledgments}

We would like to thank M. Hulsbosch, M. Beckers, Y. Verhulst and N. Wystyrk for their technical and dietary assistance throughout the study.

T.T.J.M., A.K. and J.P. designed research, S.M.M. and E.R. conducted research, S.M.M. performed statistical analysis, S.M.M. and J.P. wrote the paper, and J.P. had primary responsibility for final content. All authors have read and approved the final manuscript. 


\section{CHAPTER 6}

\section{Daily consumption of a buttermilk drink containing lutein-enriched egg-yolks does not affect postprandial metabolism or endothelial function}




\section{Abstract}

Background Previous results have shown that one-year daily consumption of a luteinenriched egg yolk containing dairy drink did not significantly affect serum lipid and lipoprotein concentrations in adults with early signs of macular degeneration.

Objective The objective of the current study was to extend our earlier finding using a broader panel of cardiovascular risk parameters in the same study population, whereby we focused on the capacity of the human body to adapt to a high fat mixed meal challenge. This was performed through evaluating changes in postprandial metabolism and endothelial function by flow-mediated dilation. These tests were performed both at the start and after one-year intervention with the lutein-enriched egg-yolk drink.

Design Subjects participated in a 1-y randomized placebo-controlled dietary intervention trial. At the start of the study, 52 subjects were included in the active (Egg) group and 49 in the control (Con) group. Changes in postprandial biochemistry (triacylglycerol (TAG), glucose and non-esterified fatty acids (NEFA)) following a mixed meal challenge were evaluated in 88 subjects; 43 in the Con and 45 in the Egg group. Flow-mediated dilation (FMD) analyses were performed in 40 subjects from the Egg group and 35 subjects from the Con group.

Results The postprandial glycemic and lipemic responses before the intervention as well as the differences in postprandial responses after one-year intervention were comparable between the Egg and the Con group. Fasting FMD was comparable between the groups before the intervention started (Egg vs. Con; $2.6 \pm 1.7 \%$ vs. $2.5 \pm 2.1 \%$, $\mathrm{P}=0.92$ ) and at the end of intervention (Egg vs. Con; $3.4 \pm 2.3$ vs. $3.1 \pm 2.4 \%, \mathrm{P}=0.57$ ). Additionally, the change in FMD following a mixed meal was comparable between the groups (Egg vs, Con; -0.8 vs $0.0 \%, P=0.33$ ).

Conclusion One-year consumption of a lutein-enriched egg yolk incorporated in a dairy drink has no effect on postprandial lipid and glucose metabolism or endothelial function as measured by FMD. 


\section{Introduction}

Eggs are an inexpensive, rich source of high-quality protein, essential fatty acids, minerals, and vitamins A, B12, D, K and folate. Additionally, eggs contain relatively high amounts of cholesterol and lutein. Lutein is a xanthophyll that is concentrated in the human retina where it plays an important role in maintaining visual function. It is not synthesized in the human body and intake depends on dietary sources such as eggs and green leafy vegetables.

Elevated intakes of other dietary components, such as cholesterol and saturated fatty acids (SAFAs), are known to increase serum LDL cholesterol concentrations (6) and as such may impair endothelial function (167). In view of the fact that an elevated intake of dietary cholesterol from eggs has been shown to increase serum LDL cholesterol (126), the intake of egg(yolks) has been discouraged over the past decades and dietary guidelines advice to limit SAFA intake to a maximum of 10en\% (125). However, evidence - mainly from large epidemiological (prospective cohort) studies - has not shown a significant relationship between egg intake and CVD $(129,130,168)$. Egg yolks are not only rich in cholesterol but also contain lutein, a xanthophyll carotenoid with antioxidant and anti-inflammatory properties. Furthermore, serum carotenoid concentrations have been inversely associated with biomarkers for cardiovascular disease risk prediction $(169,170)$ and serum asymmetric dimethylarginine (ADMA) concentrations (171). Moreover, a Chinese study recently showed that a one-year supplementation of lutein improved carotid IMT in subjects with subclinical atherosclerosis (172). Whether increased lutein intake via eggs might affect endothelial function despite the higher concomitant intake of cholesterol has not yet been investigated and was the purpose of the study described here.

Recently, we showed that one-year daily consumption of a buttermilk drink containing lutein-enriched egg-yolk did not significantly change fasting serum lipid or (apo)lipoprotein concentrations, whereas plasma lutein concentrations were markedly increased (173). However, the fact that serum cholesterol concentrations were not significantly elevated does not exclude the possibility that endothelial function might have been affected. Evidence for this statement comes from earlier studies in mice that reported a decreased endothelial function in animals fed a high-cholesterol diet despite the fact that circulating serum cholesterol concentrations were hardly affected (174). Therefore, although fasting lipid profiles are of great value in CVD risk predictions we here further evaluated the effects on a broader panel of cardiovascular risk parameters in the same study population before and after one-year daily consumption of luteinenriched egg-yolks incorporated in a dairy drink. Moreover, we measured the capability of the body to adapt to a high fat mixed meal challenge by evaluating changes in 
postprandial lipid and glucose metabolism and endothelial function, measured by flowmediated dilation.

\section{Subjects and methods}

\section{Subjects}

One hundred and one ( $\mathrm{N}=101)$ subjects were recruited for a one-year double blind placebo-controlled intervention trial with a parallel design, which was approved by the Medical Ethics Committee of Maastricht University Medical Centre and registered at clinicaltrials.gov as NCT00902408. Subjects were recruited via advertisements in newspapers in the province of Limburg, The Netherlands. The trial adhered to the tenets of the declaration of Helsinki and before the screening procedure started, written informed consent was obtained from all subjects. The main inclusion criteria were being at least 50 years of age, not receiving treatment for diabetes or elevated serum cholesterol concentrations and having early signs of macular degeneration, as evidenced by drusen and / or retinal pigment epithelium alterations in at least one eye assessed through fundus photography. Next to this, it was not allowed to use dietary supplements containing lutein or zeaxanthin. Subjects allergic to eggs or egg-products were not included.

During the 1-year follow-up of the study, 12 participants withdrew (six from the experimental group (Egg) and six from the control (Con) group). One subject that completed the study was omitted from further statistical evaluations because of outlying HDL cholesterol concentrations, as evidenced by a large Cook's distance. The flow of participants through the study and reasons for withdrawal (which were not related to the study product as such) can be found in Figure 5.1.

$\begin{aligned} & \text { Table } 6.1 \text { Nutrient composition of control- and lutein-enriched egg-yolk } \\
& \text { drink (per daily } 80 \mathrm{~mL} \text { dosage) }\end{aligned}$
\begin{tabular}{lcc} 
Control drink & $\begin{array}{c}\text { Lutein-enriched } \\
\text { egg-yolk drink }\end{array}$ \\
\hline Energy (kcal) & 41 & 131 \\
Fat (g) & 0.4 & 9.2 \\
SFA (\%) & 67.0 & 32.5 \\
TransFA (\%) & 3.1 & 0.3 \\
MUFA (\%) & 26.2 & 49.5 \\
PUFA (\%) & 3.7 & 17.7 \\
Protein (g) & 2.7 & 6.2 \\
Carbohydrate (g) & 6.6 & 5.8 \\
Cholesterol (mg) & 2.0 & 323 \\
Lutein (mg) & - & 1.4 \\
Zeaxanthin (mg) & - & 0.3
\end{tabular}


TABLE 6.2 Nutrient composition of muffins (serving of 2)

\begin{tabular}{ll} 
& Muffins \\
\hline Energy, kJ & 4095 \\
Energy, kcal & 980 \\
Protein, g & 14.5 \\
Carbohydrates, g & 103.0 \\
Total fat, g & 56.6 \\
SFA, g & 33.9 \\
TransFA, g & 2.2 \\
MUFA, g & 14.5 \\
PUFA, g & 2.7 \\
Cholesterol, mg & 349 \\
\hline
\end{tabular}

\section{Study design and products}

The study had a randomized, double blind, placebo-controlled parallel design with a duration of one year. Subjects were randomly assigned to either the Con or Egg group before starting the study. The intervention consisted of a lutein-enriched egg-yolk based dairy drink (provided by Newtricious R\&D, Oirlo, The Netherlands). Egg-yolks were enriched through natural sources, via the feed of laying hens. This feed complied with the EU regulation concerning the maximal xanthophyll level of $80 \mathrm{ppm}$. One and a half enriched egg-yolks were then incorporated into an $80 \mathrm{~mL}$ buttermilk drink that provided on average $1.4 \mathrm{mg}$ lutein and $323 \mathrm{mg}$ cholesterol per day. Subjects allocated to the Con group received a comparable buttermilk drink, without the addition of egg yolks. The color of the control drink was matched to the lutein-enriched egg drink through addition of synthetic colorants E104 and E110. The nutrient composition of both drinks can be found in Table 6.1.

Subjects participated in a mixed meal challenge at the start of the study and again after one-year consumption of either the control or lutein-enriched egg-drinks. The highfat mixed meal consisted of two buttercup cakes, with a total macronutrient composition as presented in Table 6.2. One batch of muffins was prepared for the entire study and stored at $-20^{\circ} \mathrm{C}$ until consumption. On the postprandial test day, subjects arrived at university after an overnight fast. First an FMD measurement was performed, followed by insertion of an intravenous cannula (Becton, Dickinson and Company, Franklin Lanes, NY, USA) in an antecubital vein and collection of a fasting (TO) blood sample. Hereafter, subjects were asked to consume the mixed meal within $10 \mathrm{~min}$. Subsequent blood samples were drawn 15 (T15), 30 (T30), 45 (T45), 60 (T60), 90 (T90), 120 (T120), and 240 (T240) min after mixed meal consumption. Subjects were allowed to drink water at their convenience throughout the entire postprandial test. 


\section{Flow-mediated dilation}

Before and $4 \mathrm{~h}$ after consuming the mixed meal, FMD measurements were performed according to standard guidelines (17) to assess endothelium-dependent vascular reactivity. The same ultrasonographer performed all measurements by using a highresolution ultrasound system with a $3-11 \mathrm{MHz}$ wide band linear array transducer (Philips Sonos 5500 System; Philips Ultrasound, Andover, MA, USA). After at least 15-min supine rest in a quiet, temperature-controlled room, images of the brachial artery were taken longitudinally, 2-10 cm above the antecubital fossa in combined Doppler / brightness mode (B-mode). A probe holder was used to secure the position of the transducer during the measurement. After a $3 \mathrm{~min}$ baseline measurement, a peripherally hypoxic state was induced by inflation of a pneumatic tourniquet (Hokanson TD312 automatic cuff inflator, D.E. Hokanson, Inc. WA, USA) placed around the forearm, inflated to a pressure of at least $50 \mathrm{mmHg}$ above systolic pressure, with a minimal pressure of 200 $\mathrm{mmHg}$. After 5 minutes, the cuff was deflated, resulting in reactive hyperemia, which was recorded for another 5 minutes. Images were recorded on DVD continuously during the entire $13 \mathrm{~min}$ measurement protocol. Acquired images were analyzed using an inhouse developed semi-automated image-analysis algorithm (Dept. of Biomedical Engineering, Maastricht University Medical Center, The Netherlands). FMD response was expressed as the percentage increase in brachial artery diameter from baseline to maximal dilation post-occlusion.

\section{Blood sampling and analyses}

Blood was sampled into NaF-containing tubes and serum separator tubes (Becton, Dickinson and Company, Franklin Lanes, NY, USA) at the indicated time points, which were in between the two FMD measurements at baseline and after 240 minutes. NaFcontaining tubes were immediately placed on ice after blood sampling, followed by centrifugation at $1300 \times \mathrm{g}$ for $15 \mathrm{~min}$ at $4^{\circ} \mathrm{C}$. Serum separator tubes were allowed to clot for at least $30 \mathrm{~min}$ at $21^{\circ} \mathrm{C}$ before centrifugation at $1300 \mathrm{xg}$ for $15 \mathrm{~min}$ at $21^{\circ} \mathrm{C}$. Plasma and serum aliquots were directly snap-frozen in liquid nitrogen and stored at $-80^{\circ} \mathrm{C}$ until analysis.

Plasma glucose concentrations were measured in NaF plasma at T0, 15, 30, 45, 60, 90, 120 and 240 (Horiba ABX, Montpellier, France). Non-esterified fatty acid (NEFA) concentrations were also analyzed in NaF plasma (NEFA kit; WAKO Chemicals, Germany) at the indicated time points. Concentrations of TAG, corrected for free glycerol, were determined in serum samples from T0, 15, 30, 45, 60, 120 and 240 (GPO Trinder; Sigma Diagnostics). 
Fasting serum samples were analyzed for total cholesterol, HDL cholesterol, TAG and apolipoprotein A-I (apoA1) and apolipoprotein B100 (apoB100) concentrations. LDL cholesterol concentrations were calculated according to the Friedewald equation (90).

\section{Statistical analyses}

All results are presented as mean \pm SD, unless otherwise indicated. Differences in baseline concentrations as well as changes in metabolic risk parameters and FMD between the Egg and Con group were tested using an independent samples student's ttest. Pearson correlation coefficients were used to calculate correlations. Postprandial glucose, TAG and NEFA changes were assessed by calculating differences within a subject between baseline and the end of intervention for each postprandial time point. Differences between Egg and Con group were assessed by linear mixed model (LMM) analyses with treatment and time point as within-subject fixed factors and treatment $x$ time as interaction term. If the interaction term was not significant, it was omitted from the model. If factor time was significant, post hoc tests with Bonferroni correction were carried out to compare concentrations to baseline concentrations. Change between FMD responses before and after a mixed meal at baseline were distracted from the response before and after a mixed meal after the one-year intervention. Differences between these responses were evaluated by an independent samples student's T-test. Differences were considered statistically significant if $P<0.05$. All statistical analyses were performed using SPSS 21.0 for Mac Os X (SPSS Inc., Chicago, IL, USA). 
Table 6.3 Baseline characteristics, serum lipids and (apo)lipoproteins, markers for cholesterol metabolism and liver- and kidney function of study participants within the egg-drink (Egg) and control (Con) groups1

\begin{tabular}{|c|c|c|c|c|c|c|}
\hline \multirow[b]{2}{*}{$\operatorname{Sex}(M / F)$} & \multicolumn{3}{|c|}{$\begin{array}{c}\text { Egg } \\
(\mathrm{N}=45)\end{array}$} & \multicolumn{3}{|c|}{$\begin{array}{c}\text { Con } \\
(\mathrm{N}=43)\end{array}$} \\
\hline & 15 & / & 30 & 14 & / & 29 \\
\hline Age, y & 62 & \pm & 6 & 62 & \pm & 8 \\
\hline Smoking, yes/no ${ }^{2}$ & 18 & / & 26 & 16 & / & 27 \\
\hline $\mathrm{BMI}, \mathrm{kg} / \mathrm{m}^{2}$ & 26.9 & \pm & 3.6 & 26.3 & \pm & 3.7 \\
\hline Systolic blood pressure, $\mathrm{mmHg}$ & 133 & \pm & 18 & 136 & \pm & 23 \\
\hline Diastolic blood pressure, $\mathrm{mmHg}$ & 76 & \pm & 9 & 78 & \pm & 11 \\
\hline Flow-mediated dilation, $\%^{3}$ & 2.48 & \pm & 1.74 & 2.59 & \pm & 2.20 \\
\hline Serum total cholesterol, $\mathrm{mmol} / \mathrm{L}$ & 6.01 & \pm & 1.14 & 6.04 & \pm & 1.15 \\
\hline Serum LDL cholesterol, mmol/L & 3.82 & \pm & 1.06 & 3.87 & \pm & 0.98 \\
\hline Serum HDL cholesterol, mmol/L & 1.65 & \pm & 0.41 & 1.67 & \pm & 0.39 \\
\hline Serum apoB100, g/L & 0.99 & \pm & 0.24 & 0.99 & \pm & 0.22 \\
\hline Serum apoAl, g/L & 1.38 & \pm & 0.21 & 1.38 & \pm & 0.21 \\
\hline Serum TAG, mmol/L & 1.18 & \pm & 0.63 & 1.09 & \pm & 0.45 \\
\hline Plasma glucose, $\mathrm{mmol} / \mathrm{L}$ & 5.46 & \pm & 0.43 & 5.41 & \pm & 0.46 \\
\hline Serum hsCRP, mg/L & 2.49 & \pm & 4.13 & 1.96 & \pm & 2.17 \\
\hline Plasma NEFA, $\mu \mathrm{mol} / \mathrm{L}$ & 503 & \pm & 167 & 518 & \pm & 209 \\
\hline Serum creatinine, $\mu \mathrm{mol} / \mathrm{L}$ & 70.8 & \pm & 13.4 & 75.0 & \pm & 12.4 \\
\hline Serum ALP, IU/L & 74.9 & \pm & 16.6 & 84.3 & \pm & $22.6^{*}$ \\
\hline Serum GGT, IU/L & 28.2 & \pm & 16.7 & 24.7 & \pm & 12.6 \\
\hline Serum ASAT, IU/L & 19.6 & \pm & 7.2 & 22.6 & \pm & 12.4 \\
\hline Serum ALAT, IU/L & 21.6 & \pm & 6.6 & 23.9 & \pm & 12.6 \\
\hline Serum bilirubin, $\mu \mathrm{mol} / \mathrm{L}$ & 15.0 & \pm & 4.5 & 16.6 & \pm & 7.1 \\
\hline
\end{tabular}

${ }^{1}$ Values are means \pm SDs. *Different from Con, $P<0.05$. ALAT; Alanine transaminase, ALP; Alkaline phosphatase, ASAT; Aspartate transaminase, CRP; high-sensitivity Creactive protein, GGT; Gamma-glutamyltranspeptidase, NEFA; non-esterified fatty acids.

${ }^{2}$ Smoking status of one subject in the Egg group was not provided

${ }^{3}$ Flow-mediated dilation results are shown for $\mathrm{N}=42$ in the Egg group and $\mathrm{N}=39$ in the Con group 


\section{Results}

\section{Study participants}

The flow of participants throughout the study including reasons for drop out is shown in Figure 5.1. Baseline characteristics of the eighty-nine subjects that completed the study are shown in Table 6.3. One subject was omitted from final analyses because of outlying HDL cholesterol concentrations. Therefore, results in metabolic parameters are shown for eighty-eight subjects ( $N=45$ in the Egg and $N=43$ in the Con group). Within this group of eighty-eight subjects, there were technical problems during the FMD measurement on one of the test-days, which hampered proper analysis in five subjects (one in the Con group and four in the Egg group). In addition, eight subjects (four in both groups) were omitted from the statistical analysis on FMD, because of poor quality of the FMD measurements. Therefore, FMD results are shown for seventy-five subjects ( $N=40$ in the Egg and $\mathrm{N}=35$ in the Con group).

\section{Fasting lipids, (apo)lipoproteins and FMD}

As described earlier, no significant changes were found in fasting serum total-, HDL- and LDL cholesterol, total-to-HDL cholesterol ratio, apoB100, apoAI, TAG and NEFAs between the Egg and Con group over the 1-year intervention (173). As shown in Figure 6.1, no significant difference was found in fasting FMD between the groups at the start of intervention (Egg vs. Con; $2.6 \pm 1.7 \%$ vs. $2.5 \pm 2.1 \%, \mathrm{P}=0.92$ ). Moreover, there were no significant correlations between any of the metabolic parameters and FMD (data not shown).

\section{Postprandial glycaemia and lipemia}

Fasting concentrations of NEFA, glucose and TAG were not different between the Con and the Egg group (Figure 6.2). Glucose and TAG concentrations increased and NEFA decreased significantly after mixed meal consumption, both before and after intervention ( $P<0.001$ for time effect). The change in postprandial glucose, NEFA and TAG was not different between the Egg and Con group after one year of consuming the lutein-enriched egg drink. However, a significant difference between the Egg and Con group regarding the factor time was found for glucose and NEFA ( $P=0.027, P=0.002$ ). This implies that these postprandial responses are different between both groups, irrespective of the intervention. Post-hoc comparisons did not reveal which time point was exactly different from baseline in either the glucose or the NEFA response. 

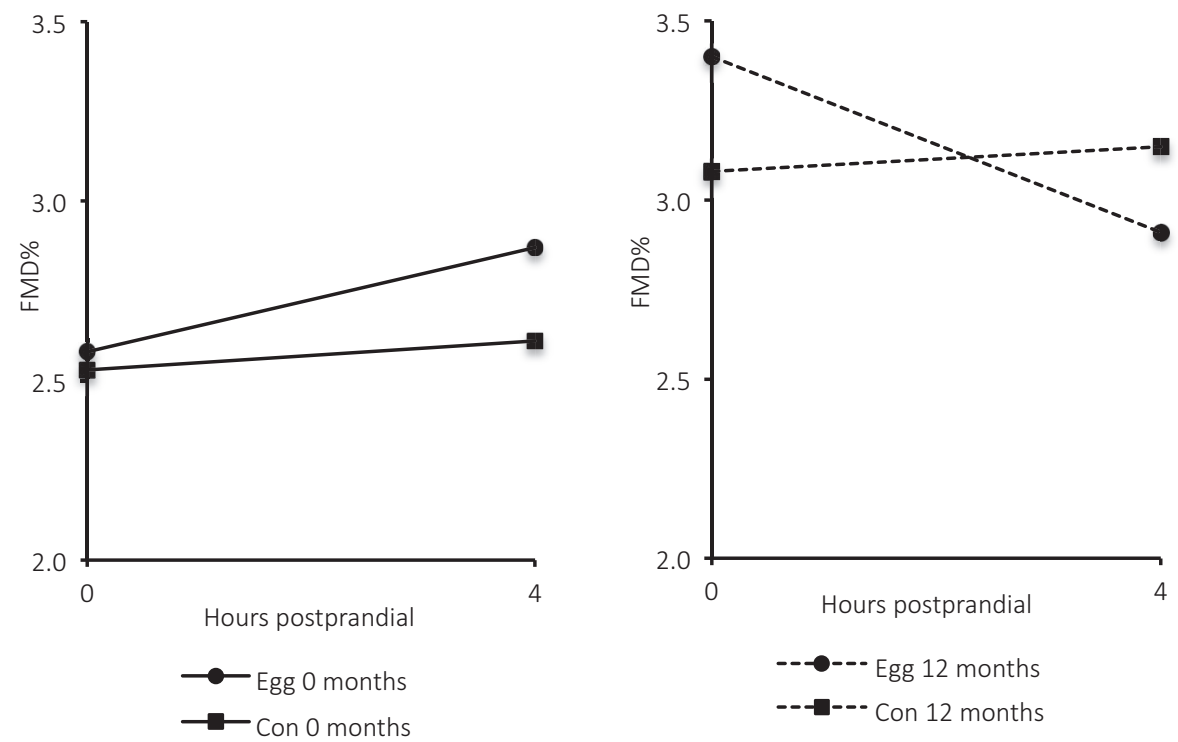

\begin{tabular}{|c|c|c|c|c|c|c|}
\hline FMD\% & Con & & & Egg & & \\
\hline & Fasting & $\begin{array}{l}\text { 4h post- } \\
\text { prandial }\end{array}$ & $\begin{array}{l}\text { Post- } \\
\text { prandial } \\
\text { change }\end{array}$ & Fasting & $\begin{array}{l}\text { 4h post- } \\
\text { prandial }\end{array}$ & $\begin{array}{l}\text { Post- } \\
\text { prandial } \\
\text { change }\end{array}$ \\
\hline Start intervention & $2.5 \pm 2.1$ & $2.6 \pm 2.1$ & $0.1 \pm 1.7$ & $2.6 \pm 1.7$ & $2.9 \pm 2.3$ & $0.3 \pm 2.6$ \\
\hline $\begin{array}{l}\text { End intervention } \\
\text { Fasting }\end{array}$ & $3.1 \pm 2.4$ & $3.2 \pm 1.9$ & $0.1 \pm 2.6$ & $3.4 \pm 2.3$ & $2.9 \pm 1.7$ & $-0.5 \pm 2.5$ \\
\hline change & $0.5 \pm 3.1$ & & & $0.8 \pm 2.9$ & & \\
\hline
\end{tabular}

Figure 6.1 Change in FMD\% measured at the start of intervention ( 0 months, solid lines) and at the end of intervention (12 months, dashed lines) in the Egg group (rounds) and in the Con group (squares). No significant differences were found. Exact FMD values are given in the accompanying table. 

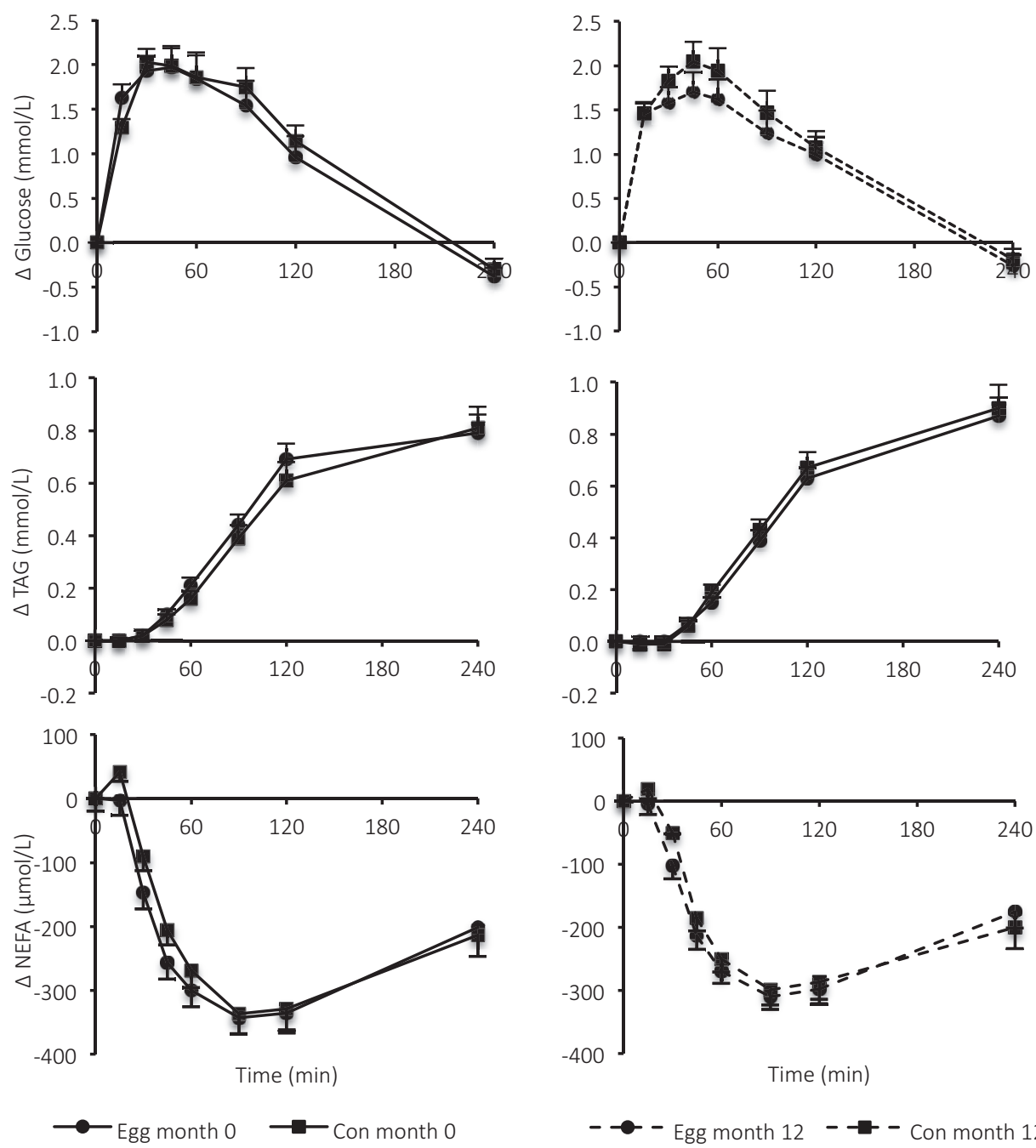

- - Egg month $12-\rightarrow-$ Con month 12

Figure 6.2. Mean changes ( \pm SEM) in plasma glucose, serum triacylglycerol (TAG) and non-esterified fatty acid (NEFA) concentrations following a mixed meal at the start of intervention (month 0 ) and at the end of intervention (month 12) in a parallel study in subjects with early signs of macular degeneration. Analyses were performed in 45 subjects in the Egg group and 43 subjects in the Con group. Differences between Egg and Con groups were analyzed using Linear Mixed Models. There were no significant meal $x$ time interactions for NEFA, TAG and glucose. Time effects were $\mathrm{P}<0.05$ for NEFA and glucose. No effects of intervention were found.

\section{Postprandial FMD}

At the start of intervention, the postprandial change in FMD was not significantly different between the groups ( $0.1 \pm 1.7 \%$ in the Con and $0.3 \pm 2.6 \%$ in the Egg group). 
After 12 months of intervention, fasting FMD showed a non-significant absolute increase of $0.5 \pm 3.1 \%$ in the Con group and $0.8 \pm 2.9 \%$ in the Egg group compared to baseline. Also, no difference in postprandial FMD between the Egg $(-0.5 \pm 2.5 \%)$ and Con $(0.1 \pm$ 2.6\%) was found. Most importantly, the change in FMD after a mixed meal before and after one-year intake of lutein-enriched egg-drinks was not different from the controls receiving a placebo dairy drink (Egg vs. Con; $-0.8 \pm 3.3$ vs. $0.0 \pm 3.5 \%, P=0.33$ ).

\section{Discussion}

This study shows that during a period of one-year, daily consumption of a dairy drink containing 1.5 lutein-enriched egg-yolks, does not affect endothelial function as measured by flow-mediated dilation. Moreover, consuming this dairy drink did not alter postprandial glycemic and lipemic responses.

Over the past decades, the effect of dietary cholesterol in general and egg consumption in particular on cardiovascular outcomes has been a controversial issue. The reason for potential concerns is the well-defined serum LDL cholesterol raising effect of increased dietary cholesterol intake (126). As shown earlier, an increase in LDL cholesterol concentration of $0.16 \pm 0.01 \mathrm{mmol} / \mathrm{L}$ was expected after 1 -y consumption of the lutein-enriched egg-drink (173). In a healthy population, with average serum LDL cholesterol concentrations of $3 \mathrm{mmol} / \mathrm{l}$, this means an increase in serum LDL cholesterol of approximately 5\%. Based on estimations as described in a meta-analysis from the Cholesterol Treatment Trialists' Collaboration, this would translate in an increased risk for major vascular events of $\pm 5 \%$ (175). However, epidemiologic studies evaluating the effect of egg consumption on (intermediary) outcomes of CVD are not conclusive (129, $130,168,176,177)$. Moreover, the long-term effect of increased dietary cholesterol intake on intermediate cardiovascular endpoints has not been assessed in a randomized clinical trial. Endothelial dysfunction, as assessed by FMD in the present study, is an early hallmark in the process of atherosclerotic plaque development (178), which therefore might act as intermediate cardiovascular endpoint. Earlier studies did not observe an effect of short-term daily intake of eggs on endothelial function and lipid profile in both healthy (179) and hyperlipidemic (180) subjects. Our results extend these findings and show that also long-term consumption of lutein-enriched egg yolks incorporated in a buttermilk drink had no effect on endothelial function as measured by FMD in a population of slightly hypercholesterolemic, overweight male and female subjects with early signs of macular degeneration.

It should be mentioned that the egg yolks used in this study were enriched in lutein, a carotenoid that is hypothesized to be protective against early atherosclerosis (165). An in vitro study revealed that lutein was able to improve endothelial function through 
increasing NO and decreasing the release of endothelin (181). Furthermore, lutein downregulated NF-KB signaling, and decreased several markers for endothelial activation and inflammation. Also, a significant inverse relation was observed between plasma soluble intercellular adhesion molecule-1 (sICAM-1) and lutein concentrations in a crosssectional study in a general population sample (182). Recently, a study from China showed that one-year daily supplementation of $20 \mathrm{mg}$ lutein improved carotid IMT in subjects with subclinical atherosclerosis (172). Therefore, it might be possible that the combined intake of dietary cholesterol with an increased quantity of lutein counterbalanced each other's effects on flow-mediated dilation. This also implies that the observation that one year daily intake of these lutein-enriched egg-yolks did not affect endothelial function cannot be extrapolated to regular eggs of which the yolks are not enriched in lutein. In view of the difficulties in obtaining a large sample size of individuals with age-related macular degeneration we decided not to include a reference group that consumed regular eggs for one year in our trial. However, the primary goal of our trial was to find out whether intake of these lutein enriched eggs were able to increase circulating lutein levels without showing an adverse effect on CVD risk parameters and as such our study achieved its goals. The effects of the daily intake of lutein-enriched egg-yolks on visual function will be addressed in a separate paper (manuscript in preparation).

Another explanation for not finding an effect on endothelial function, after consuming one-and-a half egg yolks incorporated in a buttermilk drink that provided on average $323 \mathrm{mg}$ dietary cholesterol additionally per day for one year, might be caused by the fact that egg consumption is thought to mainly increase particularly the larger, more buoyant, LDL (LDL-1) particles. These particles are thought to be less atherogenic than small (LDL-3+) particles, because, compared to smaller LDL particles, they are not able to enter the arterial wall as easily, their binding capacity to proteoglycans is not enhanced, they are less susceptible to oxidation and their affinity to bind to the LDL receptor is not decreased (183). These features might explain the fact that small LDL particles are related to endothelial dysfunction in several groups of subjects (184-186), which is not the case for larger LDL particles. However, LDL subclass distribution was not measured in our subjects.

In conclusion, we here show that daily consumption of a dairy drink containing lutein-enriched egg-yolks had no adverse effect on endothelial function or postprandial glucose and lipid metabolism in subjects with early signs of macular degeneration. Our findings support the use of this lutein-enriched egg-yolk drink as a natural source of lutein.

\section{Acknowledgments}

We would like to thank M. Hulsbosch, M. Beckers, Y. Verhulst and N. Wystyrk for their technical and dietary assistance throughout the study. 
T.T.J.M.B., A.K. and J.P. designed the research; S.M.v.d.M. conducted research; S.M.v.d.M. performed the statistical analysis, S.M.v.d.M. and J.P. wrote the first draft of the paper, which was further edited by T.T.J.M.B and A.K. ; J.P. had primary responsibility for final content. All authors read and approved the final manuscript. 


\section{CHAPTER 7}

General Discussion 


\section{Introduction}

The metabolic syndrome (MetS) is a complex multifactorial disorder, of which the incidence is increasing worldwide. Several strategies to improve risk factors associated with the metabolic syndrome are used including lifestyle modifications. These lifestyle modifications aim, for instance, at increasing physical exercise, quitting smoking, adapting to a healthy diet and losing weight, which are of utmost importance to lower the risk for cardiovascular disease (CVD) and type 2 diabetes mellitus (T2DM). The research presented in this thesis focused on two well-defined nutritional intervention studies that were designed to assess the effects on metabolic indices related to CVD. Especially, effects on postprandial metabolism and endothelial function were examined.

\section{Nutritional strategies to interfere with the metabolic syndrome}

The metabolic profile can be improved through adapting to nutritional strategies that specifically target one or more characteristics of the MetS such as a disturbed lipid metabolism, blood pressure or insulin sensitivity. The most well-known dietary strategies to interfere with the MetS and its associated metabolic abnormalities include (a) manipulation of dietary macronutrient intake, for example adhering to a high-protein diet, (b) the intake of functional foods and bioactive nutrients and (c) adherence to specific dietary patterns, such as the Mediterranean diet (187).

The relation between the intake of bioactive ingredients and markers for cardiovascular health is described in this thesis. A dietary component that is thought to exhibit beneficial effects on cardiovascular health is discussed in chapter 2. In that chapter we focused on the role of polyphenols in dyslipidemia and endothelial function. Several short-term studies ( $<2$ weeks) have described that flavonoids from cocoa had a beneficial effect on LDL cholesterol concentrations. However, longer-term studies (4-12 weeks) could not confirm this finding, which raised questions on the clinical usefulness of these compounds in dyslipidemia. Several studies described a positive relation between the intake of flavonoids from dark chocolate and improved endothelial function, as summarized in a systematic review and meta-analysis (53). This even led to approval of a so-called 'new function (13.5)' health claim by the European Food Safety Authority (EFSA) regarding the positive relation between the intake of cocoa flavanols and maintenance of endothelium-dependent vasodilation (76).

As this example shows, a bioactive ingredient has the potency to play an important role in lowering the risk for CVD and improving several characteristics of the metabolic syndrome. In this thesis we examined the relation between two different nutritional compounds and the risk for markers related to the MetS and CVD into great detail. The first study examined the effects of 28 days of trans-resveratrol intake on metabolic 
health outcomes. Trans-resveratrol is thought to beneficially influence lipid and lipoprotein metabolism, mainly based on in vitro and animal studies. The second study was a one-year dietary intervention trial that focused on the effects of the intake of a lutein-enriched egg yolk based buttermilk drink on lipid and lipoprotein metabolism. Although the drink provided an increased amount of lutein, daily dietary cholesterol intake from it exceeded 300 mg, which is the upper intake limit defined by the American Health Association (124). In both studies, not only the effects on cardiovascular risk markers in the fasting state was evaluated, but subjects also participated in a dietary challenge test in order to study the effect of the intervention on postprandial metabolism and consequent changes in endothelial function.

\section{Trans-resveratrol and fasting metabolic risk markers}

The first study described in this thesis (chapter 3) was performed in overweight and slightly obese subjects with low HDL cholesterol concentrations. The primary objective of this study was to assess the influence of 4-weeks $150 \mathrm{mg}$ trans-resveratrol intake on apolipoprotein A-I concentrations. ApoA-I is the major protein constituent of the HDL particle. Besides this, effect on fasting metabolic risk markers for lipid and lipoprotein metabolism, glucose metabolism and inflammation and endothelial function were assessed. Based on literature, a positive effect of trans-resveratrol intake on apoA-I metabolism was hypothesized. Although in vitro and animal studies have shown positive effects of resveratrol on genes and proteins that are involved in lipid and glucose metabolism $(83,84,88,188)$, in our study, no effects of 4-wk trans-resveratrol intake on markers related to cardiovascular health were found.

As mentioned, this study focused on the potential of resveratrol to elevate serum apoA-I concentrations. Subjects with higher serum HDL cholesterol concentrations were shown to have a lower cardiovascular risk in epidemiological studies $(32-34,77)$. However, increasing HDL cholesterol concentrations was not related to improved cardiovascular outcomes in studies examining pharmacological interventions such as high-dose niacin or cholesteryl ester transfer protein (CETP) inhibitors (38, 39, 78, 189). In our trans-resveratrol study, increasing the serum apoA-I concentration was the primary outcome, which may be a more important target to reduce the risk for major cardiovascular events than increasing serum HDL cholesterol concentrations (35). ApoA-I may play a crucial role in many of the favorable effects attributed to HDL particles, such as its anti-inflammatory, anti-thrombotic and anti-oxidative effects $(36,37)$.

Although trans-resveratol did not increase serum apoA-I and HDL cholesterol concentrations in our study, this does not fully exclude the possibility that transresveratrol improves metabolic risk markers related to cardiovascular health. Cholesterol efflux capacity could be measured in future studies to assess the effect of transresveratrol on HDL metabolism. Cholesterol efflux from macrophages to HDL is the 
critical initial step in reverse cholesterol transport, which has been linked to the prevention of atherosclerosis in animal models. Cholesterol efflux capacity is a biomarker that symbolizes a key step in reverse cholesterol transport. Thus, cholesterol efflux capacity might be a valuable parameter to assess the effect of trans-resveratrol intake on CVD risk reduction. Recently, an inverse relation between the incidence of cardiovascular events and cholesterol efflux capacity was found (190). Also, other methods to measure HDL functionality receive more and more attention. Functional roles of $\mathrm{HDL}$ include antimicrobial, antioxidant, anti-glycation, anti-inflammatory, antithrombotic, antiplatelet and immune modulatory activities (191). In vitro studies show a positive effect of resveratrol on $\operatorname{HDL}$-mediated efflux $(59,192,193)$, while this effect has not yet been established in vivo.

In contrast to the results found in our study, a recent paper shows that high-dose (1000 mg in week 1 and $2000 \mathrm{mg}$ in week 2) resveratrol intake reduced intestinal and hepatic lipoprotein particle production in eight overweight and obese, mildly hypertriglyceridemic men (93). Compared to our study, this study had a shorter duration, the intake of resveratrol was higher and a smaller group of male subjects was included. Also, triglyceride-rich lipoprotein kinetics was measured, which might be interesting for future research in this field. Additionally, in order to evaluate the clinical benefits of resveratrol in this particular group of subjects, a longer-term study should be performed.

Besides the effects of trans-resveratrol intake on lipid and lipoprotein metabolism, its effect on fasting glucose concentrations and markers for low-grade inflammation were studied. However, we only found a tendency towards decreased fasting plasma glucose concentrations, whereas we did not find a difference in $\mathrm{HOMA}_{\mathrm{R}}$ between the trans-resveratrol and control periods. In addition, we did not find an effect of transresveratrol on soluble IL-6, TNF $\alpha$, E-selectin, thrombomodulin, P-selectin, ICAM-3, ICAM1 and VCAM-1 concentrations.

To summarize, although it has been postulated that trans-resveratrol is a promising dietary ingredient to influence characteristics of the metabolic syndrome, no effects were found on fasting apoA-I, HDL cholesterol concentrations and other metabolic risk markers related to cardiovascular health, such as glucose concentration and inflammatory parameters. Future research could study HDL functionality and lipoprotein production by the liver and intestine into more detail, for instance through measuring cholesterol efflux capacity and stable isotope infusion, in order to further map the relation between trans-resveratrol intake and CVD risk. 
Lutein-enriched egg yolks incorporated into a buttermilk drink and fasting metabolic riskmarkers

The second study described in this thesis evaluated the possible negative effects of oneyear daily consumption of a buttermilk drink that contained one and a half luteinenriched egg yolk thereby providing $300 \mathrm{mg}$ of cholesterol, on serum lipid and lipoprotein concentrations (chapter 5). The study was primarily designed to assess the effects of this buttermilk drink on visual function, macular pigment optical density (MPOD) and plasma lutein concentrations in adults with early signs of age-related macular degeneration (AMD), as an increased intake of lutein and zeaxanthin is proposed to have a preventive effect in this most frequent cause of blindness in elderly in the Western world (194). Lutein bioavailability is higher from egg-yolks than from supplements and spinach, at least in a small-scale but well-controlled study in ten healthy subjects (133). This might be explained by the fact that lutein is located in the digestible lipid matrix within the egg-yolk, which is composed of cholesterol, triacylglycerols, and phospholipids (195), which leads to improved lutein uptake via enterocytes in the gastrointestinal tract. As the debate on the effect of frequent egg consumption on cardiovascular risk is still ongoing, our main aim was to assess the effect of 1-year daily consumption of lutein-enriched egg-yolks incorporated in a buttermilk drink on lipid- and lipoprotein metabolism in order to estimate the effect of consuming this dairy drink on cardiovascular risk.

Although we hypothesized a negative effect of consuming the lutein-enriched egg drink on lipid- and lipoprotein metabolism, consumption of this drink for one year did not result in significantly increased serum lipids and lipoproteins. The non-significant increases in total and LDL cholesterol concentration were completely in line with prediction equations, although these predictions were based on studies with a maximum duration of 20 weeks (126), whereas our study had a duration of one year. Altogether, daily consumption of the lutein-enriched egg drink for one year did not result in significant increases in lipid and (apo)lipoprotein concentrations. Although the risk of cardiovascular events caused by the non-significant increase in total and LDL cholesterol and decrease in HDL cholesterol after consumption of this drink should be kept in mind, it needs to be emphasized that the egg-yolks in this drink were enriched in lutein, which might be beneficial to (cardiovascular) health (165).

Another goal of this study was to assess the effects of consuming the luteinenriched buttermilk drink in specified subgroups, which touches upon the concept of personalized nutrition. Personalized nutrition takes into consideration each individual's physical and genetic make-up in order to tailor one's personal diet to his or her genetic make-up. It is related to the concept of 'nutritional genomics and genetics' that studies gene and nutrient interactions. It is the ultimate aim to design diets that are optimal in maintaining health and preventing diseases on an individual basis (196). In our study, markers for cholesterol metabolism were assessed in subjects with early signs of AMD in 
order to classify subjects as being a cholesterol synthesizer (low campesterol-tolathosterol ratio) or a cholesterol absorber (high ratio). From these sub-group analyses, we were able to identify a subpopulation of cholesterol absorbers that had higher HDL cholesterol concentrations at the start of the 12-month intervention, but yet showed a more pronounced increase in serum HDL cholesterol concentration, which is of particular interest since HDL particles are thought to be important in lutein transport $(135,197)$. Moreover, these subjects had a more pronounced increase in plasma lutein concentrations, compared to individuals classified as cholesterol synthesizers. The increase in serum cholesterol was comparable in the group classified as cholesterol absorbers as compared to the group classified as cholesterol synthesizers. Based on this, persons classified as cholesterol absorber seem to be the ideal target population for starting the intake of a lutein-enriched egg-drink to prevent the development of AMD, because here, lutein is provided within a cholesterol-rich matrix.

In addition, apolipoprotein E (APOE) genotype was assessed in this study, because of the well-known relation between APOE genotype and cholesterol metabolism and the suggested association between APOE genotype and the risk of developing age-related macular degeneration (144-146). A pooled analysis of 15 studies provided evidence for a protective role of the APOع4 haplotype in AMD, while the $\varepsilon 2 \varepsilon 2$ diplotype was associated with an increased risk for late AMD. Moreover, the $\varepsilon 4$ isoform is related to a higher cholesterol absorption efficiency, while the $\varepsilon 2$ isoform is associated with lower fractional intestinal cholesterol absorption (146). Individuals in the APOE2 group would thus be an interesting target group for this intervention, as they are characterized by low plasma lutein concentrations, while this group is not expected to have a strong increase in total or LDL cholesterol concentrations. However, no significant differences between the APOE subgroups in mean changes in lipid, lipoprotein or lutein concentrations could be identified during one-year lutein-enriched egg drink consumption. This could be due to the relatively small sample size in this sub-analysis, which had a highly explorative character. Therefore, future research into this topic should be performed in larger samples.

To conclude, the intake of $300 \mathrm{mg}$ dietary cholesterol through daily consumption of a lutein-enriched egg drink based on buttermilk for one year did not increase lipid- or lipoprotein concentrations, while this approach was successful in increasing plasma lutein concentrations. A specific subgroup that could potentially benefit most from this dairy drink is a group that can be specified as cholesterol absorbers, who showed a significant higher increase in plasma lutein concentrations compared with cholesterol synthesizers. To confirm this finding, future research into these lutein-responsive subgroups should be performed in studies using a larger sample size. 
Trans-resveratrol, lutein-enriched egg yolks incorporated into a buttermilk drink and endothelial function

Another approach to determine the changes in risk for CVD induced by a specific intervention is to assess its effect on endothelial function. Flow-mediated dilation (FMD) is a widely used method to measure endothelial function, despite several shortcomings, including the fact that the measurement is technically demanding -it requires an extensive training period, specific subject preparation and is time-consuming for both the subject and the investigator-, the costly equipment and the final analysis being highly dependent on the quality of the ultrasound images (17). The beneficial characteristics of this measurement, namely the fact that it is a non-invasive method to measure NOdependent vasodilatation, which has been shown to be inversely associated with future cardiovascular events (198), make it a popular tool in clinical and nutritional research.

Trans-resveratrol has been shown to improve FMD dose-dependently after acute intake in overweight and obese adults with mildly elevated blood pressure (105). Also, 6-weeks daily $75 \mathrm{mg}$ resveratrol consumption improved FMD in healthy obese adults from $5.83 \%$ in the control period to $7.21 \%$ during resveratrol intake (98). Another study did not find an effect of daily $10 \mathrm{mg}$ trans-resveratrol intake for 3 months on FMD in 40 postinfarction patients (106). In our study, we did not find an effect of 4-week daily intake of $150 \mathrm{mg}$ trans-resveratrol in overweight male and female subjects (chapter 4). It might be that our study duration was too short, as Wong et al. had a 6-week intervention period. Furthermore, baseline characteristics of the subjects participating in both studies were not similar. Mean fasting FMD was 3.0\% after the control period in our subjects, whereas Wong et al. measured a fasting FMD of $6.6 \%$ in their subjects (98). Moreover, blood pressure was higher in our subjects $(136 / 88 \mathrm{mmHg})$ compared to blood pressure in Wong's study $(127 / 73 \mathrm{mmHg})$. It might therefore be that our subjects were too metabolically disturbed, which hampers the ability to show improvements within the 4week timeframe of our study. Next to not finding an effect on fasting FMD, we were also not able to find effects on fasting pulse wave velocity and augmentation index, which are both markers of arterial stiffness, in this study.

In the second study described in this thesis (chapter 6), FMD was measured in slightly hypercholesterolemic, overweight male and female subjects with early AMD. These subjects received lutein-enriched egg drinks or placebo for one year. FMD measurements were performed at the start and end of the intervention period. We hypothesized that consumption of this drink for one year would lead to increased serum total and LDL cholesterol concentrations, accompanied by deterioration in FMD. However, the increase of $0.15 \mathrm{mmol} / \mathrm{L}$ in serum LDL cholesterol concentrations did not reach statistical significance and no effects on fasting FMD were found. One explanation for the absence of the postulated effect could be the high concentration of lutein in the egg-yolks, which were incorporated in the dairy drink. Lutein is mainly known because of its anti-oxidant and blue light-filtering properties in the retina, where it forms the 
macular pigment (136) and plays an important role in maintaining visual function in subjects with early signs of AMD (199). Lately, the relation between carotenoids in general and lutein in particular and cardiovascular parameters has been studied extensively, both in epidemiologic $(169,171)$ and intervention studies $(172)$. In addition, an inverse relation between asymmetric dimethylarginine (ADMA), an endogenous inhibitor of endothelium nitric oxide synthase (eNOS) and as such an inhibitor of nitric oxide production in vascular endothelial cells, and serum carotenoid concentrations was found in a cross-sectional study in 470 subjects (171). A significant decrease of 0.035 $\mathrm{mm}$ in carotid artery intima-media thickness (CAIMT) -an ultrasound measurement of the thickness of the tunica media and tunica intima of the carotid artery, to detect the presence of atherosclerotic lesions- was shown in subjects with subclinical atherosclerosis that received $20 \mathrm{mg}$ of lutein daily for 12 months, leading to an inverse association between serum lutein and CAIMT (172). The aforementioned results point towards a positive relation between carotenoid status and a lower risk for CVD. Therefore, it might be that the increased lutein intake in our study counterbalanced the expected deleterious effects on FMD caused by increased dietary cholesterol intake in terms of CVD risk (125). Other groups did not find an effect of two additional eggs daily for 6-weeks on FMD in forty-nine healthy (179) or in forty hyperlipidemic male and female subjects (180). However, no long-term studies assessed the relation between increased dietary cholesterol intake and FMD.

Our results add to the idea that dietary cholesterol intake might be less detrimental in terms of CVD risk than previously thought. However, we again want to point out that the nutritional intervention used in this study also included lutein, which makes it impossible to give an advice towards CVD risk exclusively based on dietary cholesterol intake.

\section{Dietary challenge test}

In both studies described in this thesis, a dietary challenge test was introduced to be able to measure subtle changes in FMD, low-grade systemic inflammation and postprandial metabolism following the intervention periods. This approach holds a strong relation with the recently suggested change of emphasis in the definition of health from "a state of complete physical, mental and social well-being and not merely the absence of disease and infirmity" (200) towards "the ability of the human body to adapt and self-manage in the face of social, physical and emotional challenges" (201). Within this new definition, the concept of resilience links health to the capacity of the human body to cope with daily stressors that challenge homeostasis. Restoration of homeostasis is the target resultant of various physiologic systems, which were challenged by a stressor, such as a glucose or fat load. In the studies described in this 
thesis, a high fat meal was chosen as a stressor. The body's capability to adapt to a mixed meal stressor was assessed through providing the subjects with a high-fat meal. This was followed by measuring FMD -in the case of the study into the lutein-enriched egg-drinks- and FMD and arterial stiffness -in the case of the study into transresveratrol- before and 4 hours postprandially. Furthermore, glucose, insulin and triacylglycerol concentrations and inflammatory markers and markers for endothelial function were measured at specified time intervals during the postprandial phase. No effects of trans-resveratrol on postprandial glucose, insulin or triacylglycerol concentrations were observed. Also, no differences between postprandial responses in markers for endothelial activation (sE-Selectin, sICAM-1, sICAM-3, sP-Selectin, soluble thrombomodulin, sVCAM) and inflammation (IL-6, TNF $\alpha$ ) were found. Finally, no effect of the mixed meal challenge was found on FMD, pulse wave velocity (PWV) and augmentation index (Aix). Our study that evaluated the effect of one-year intake of a lutein-enriched egg-drink also did not show results on postprandially measured parameters. The postprandial response in non-esterified fatty acids, glucose and triacylglycerol were comparable between the group that consumed the egg-drink and the control group. Also, the change in FMD after a mixed meal was not different between both groups.

Although postprandial TAG concentrations increased as expected in both studies, this did not lead to the anticipated deterioration in FMD. This could be caused by the chosen time-point of FMD measurement, which was 4 hours after high-fat meal consumption. Several studies show a 4-hour postprandial FMD-deterioration after a high-fat meal $(115,116)$, while others showed this effect 2 hours postprandially $(114)$. It is therefore possible that we missed the effect on FMD because values already returned to fasting after 4 hours. Although the main triacylglycerol increase was observed within 2 hours postprandially in our studies, peak triacylglycerol concentrations were not reached within the 4-hour timeframe of the postprandial test.

\section{General conclusion and concluding remarks}

The studies described in this thesis aimed to assess the effect of two well-defined dietary interventions with trans-resveratrol and a lutein-enriched egg-yolk dairy drink on markers predicting the risk for CVD. Although trans-resveratrol was regarded a promising compound in decreasing the risk for CVD, we did not find evidence for this in our human study. A very complete set of metabolic risk markers, including (apo)lipoprotein-, glucose- and triacylglycerol concentrations, inflammatory markers and markers for endothelial function, amongst which were FMD measurements, was assessed, which were not affected by trans-resveratrol intake in this study population of overweight and slightly obese men and women. 
On the other hand, an intervention study in which subjects consumed a luteinenriched egg-yolk containing dairy drink for one year was hypothesized to induce an increase in CVD risk through the increased intake of dietary cholesterol. However, no effect on a comparable set of metabolic risk markers was found in this study either.

Future research into the relation between trans-resveratrol and metabolic risk markers should include a study duration of at least 6 weeks to exclude the possibility that study duration was the reason for not finding any relation between transresveratrol intake and metabolic risk markers in our study.

To confirm findings from the sub-group analyses performed in the study into the effect of lutein-enriched egg yolks on lipid and lipoprotein metabolism in order to identify lutein-responsive subgroups, future studies should be performed with a larger sample size.

Finally, as nutritional interventions in general are not aimed at curing people, but rather at prevention, selection of the target population should be performed with the greatest care. For instance, subjects that already suffer from the metabolic syndrome might be too far in the disease process. Therefore, future research into the relation between nutritional compounds or functional foods and the MetS should ideally be performed in subjects that are at risk for developing the syndrome, but do not suffer from it yet and thus present themselves with maximal two out of the five MetS characteristics. 
References 
1. Alberti KG, Zimmet PZ. Definition, diagnosis and classification of diabetes mellitus and its complications. Part 1: diagnosis and classification of diabetes mellitus provisional report of a WHO consultation. Diabet Med. 1998;15:539-53.

2. National Cholesterol Education Program Expert Panel on Detection E, Treatment of High Blood Cholesterol in A. Third Report of the National Cholesterol Education Program (NCEP) Expert Panel on Detection, Evaluation, and Treatment of High Blood Cholesterol in Adults (Adult Treatment Panel III) final report. Circulation. 2002;106:3143-421.

3. Alberti KG, Zimmet P, Shaw J, Group IDFETFC. The metabolic syndrome--a new worldwide definition. Lancet. 2005;366:1059-62.

4. Grundy SM, Cleeman JI, Daniels SR, Donato KA, Eckel RH, Franklin BA, Gordon DJ, Krauss RM, Savage PJ, Smith SC, Jr., et al. Diagnosis and management of the metabolic syndrome: an American Heart Association/National Heart, Lung, and Blood Institute scientific statement: Executive Summary. Crit Pathw Cardiol. 2005;4:198-203.

5. Alberti KG, Eckel RH, Grundy SM, Zimmet PZ, Cleeman JI, Donato KA, Fruchart JC, James WP, Loria CM, Smith SC, Jr., et al. Harmonizing the metabolic syndrome: a joint interim statement of the International Diabetes Federation Task Force on Epidemiology and Prevention; National Heart, Lung, and Blood Institute; American Heart Association; World Heart Federation; International Atherosclerosis Society; and International Association for the Study of Obesity. Circulation. 2009;120:1640-5.

6. Eckel RH, Alberti KG, Grundy SM, Zimmet PZ. The metabolic syndrome. Lancet. 2010;375:1813.

7. Grundy SM. Drug therapy of the metabolic syndrome: minimizing the emerging crisis in polypharmacy. Nat Rev Drug Discov. 2006;5:295-309.

8. Cameron AJ, Shaw JE, Zimmet PZ. The metabolic syndrome: prevalence in worldwide populations. Endocrinol Metab Clin North Am. 2004;33:351-75, table of contents.

9. Bos MB, de Vries JH, Wolffenbuttel BH, Verhagen H, Hillege JL, Feskens EJ. [The prevalence of the metabolic syndrome in the Netherlands: increased risk of cardiovascular diseases and diabetes mellitus type 2 in one quarter of persons under 60]. Ned Tijdschr Geneeskd. 2007;151:2382-8.

10. Lozano R, Naghavi M, Foreman K, Lim S, Shibuya K, Aboyans V, Abraham J, Adair T, Aggarwal $R$, Ahn SY, et al. Global and regional mortality from 235 causes of death for 20 age groups in 1990 and 2010: a systematic analysis for the Global Burden of Disease Study 2010. Lancet. 2012;380:2095-128.

11. Go AS, Mozaffarian D, Roger VL, Benjamin EJ, Berry JD, Blaha MJ, Dai S, Ford ES, Fox CS, Franco S, et al. Heart disease and stroke statistics--2014 update: a report from the American Heart Association. Circulation. 2014;129:e28-e292.

12. Organization WH. The European health report 2012: charting the way to well-being. Copenhagen: World Health Organization Regional Office for Europe; 2012.

13. Ross R. Atherosclerosis--an inflammatory disease. N Engl J Med. 1999;340:115-26.

14. Davignon J, Ganz P. Role of endothelial dysfunction in atherosclerosis. Circulation. 2004;109:III27-32.

15. Luscher TF, Barton M. Biology of the endothelium. Clin Cardiol. 1997;20:II-3-10. 
16. Rubbo H, Trostchansky A, Botti H, Batthyany C. Interactions of nitric oxide and peroxynitrite with low-density lipoprotein. Biol Chem. 2002;383:547-52.

17. Corretti MC, Anderson TJ, Benjamin EJ, Celermajer D, Charbonneau F, Creager MA, Deanfield J, Drexler H, Gerhard-Herman M, Herrington D, et al. Guidelines for the ultrasound assessment of endothelial-dependent flow-mediated vasodilation of the brachial artery: a report of the International Brachial Artery Reactivity Task Force. J Am Coll Cardiol. 2002;39:257-65.

18. Oh PC, Koh KK, Sakuma I, Lim S, Lee Y, Lee S, Lee K, Han SH, Shin EK. Omega-3 fatty acid therapy dose-dependently and significantly decreased triglycerides and improved flow-mediated dilation, however, did not significantly improve insulin sensitivity in patients with hypertriglyceridemia. Int J Cardiol. 2014.

19. Tousoulis D, Plastiras A, Siasos G, Oikonomou E, Verveniotis A, Kokkou E, Maniatis K, Gouliopoulos N, Miliou A, Paraskevopoulos T, et al. Omega-3 PUFAs improved endothelial function and arterial stiffness with a parallel antiinflammatory effect in adults with metabolic syndrome. Atherosclerosis. 2014;232:10-6.

20. Mancini GB, Henry GC, Macaya C, O'Neill BJ, Pucillo AL, Carere RG, Wargovich TJ, Mudra H, Luscher TF, Klibaner MI, et al. Angiotensin-converting enzyme inhibition with quinapril improves endothelial vasomotor dysfunction in patients with coronary artery disease. The TREND (Trial on Reversing ENdothelial Dysfunction) Study. Circulation. 1996;94:258-65.

21. Treasure CB, Klein JL, Weintraub WS, Talley JD, Stillabower ME, Kosinski AS, Zhang J, Boccuzzi SJ, Cedarholm JC, Alexander RW. Beneficial effects of cholesterol-lowering therapy on the coronary endothelium in patients with coronary artery disease. N Engl J Med. 1995;332:481-7.

22. Zieman SJ, Melenovsky V, Kass DA. Mechanisms, pathophysiology, and therapy of arterial stiffness. Arterioscler Thromb Vasc Biol. 2005;25:932-43.

23. Tomiyama H, Yamashina A. Non-invasive vascular function tests: their pathophysiological background and clinical application. Circ J. 2010;74:24-33.

24. Laurent S, Cockcroft J, Van Bortel L, Boutouyrie P, Giannattasio C, Hayoz D, Pannier B, Vlachopoulos C, Wilkinson I, Struijker-Boudier H, et al. Expert consensus document on arterial stiffness: methodological issues and clinical applications. Eur Heart J. 2006;27:2588-605.

25. Stehouwer CD, Henry RM, Ferreira I. Arterial stiffness in diabetes and the metabolic syndrome: a pathway to cardiovascular disease. Diabetologia. 2008;51:527-39.

26. Van Bortel LM, Laurent S, Boutouyrie P, Chowienczyk P, Cruickshank JK, De Backer T, Filipovsky J, Huybrechts S, Mattace-Raso FU, Protogerou AD, et al. Expert consensus document on the measurement of aortic stiffness in daily practice using carotid-femoral pulse wave velocity. J Hypertens. 2012;30:445-8.

27. Van Bortel LM, Duprez D, Starmans-Kool MJ, Safar ME, Giannattasio C, Cockcroft J, Kaiser DR, Thuillez C. Clinical applications of arterial stiffness, Task Force III: recommendations for user procedures. Am J Hypertens. 2002;15:445-52.

28. Mills NL, Miller JJ, Anand A, Robinson SD, Frazer GA, Anderson D, Breen L, Wilkinson IB, McEniery CM, Donaldson $\mathrm{K}$, et al. Increased arterial stiffness in patients with chronic obstructive pulmonary disease: a mechanism for increased cardiovascular risk. Thorax. 2008;63:306-11.

29. O'Rourke MF, Gallagher DE. Pulse wave analysis. J Hypertens Suppl. 1996;14:S147-57.

30. Hodis HN. Triglyceride-rich lipoprotein remnant particles and risk of atherosclerosis. Circulation. 1999;99:2852-4. 
31. Castelli WP, Garrison RJ, Wilson PW, Abbott RD, Kalousdian S, Kannel WB. Incidence of coronary heart disease and lipoprotein cholesterol levels. The Framingham Study. JAMA. 1986;256:2835-8.

32. Assmann $G$, Schulte $H$, von Eckardstein A, Huang Y. High-density lipoprotein cholesterol as a predictor of coronary heart disease risk. The PROCAM experience and pathophysiological implications for reverse cholesterol transport. Atherosclerosis. 1996;124 Suppl:S11-20.

33. Gordon T, Castelli WP, Hjortland MC, Kannel WB, Dawber TR. High density lipoprotein as a protective factor against coronary heart disease. The Framingham Study. Am J Med. 1977;62:70714.

34. Sharrett AR, Ballantyne CM, Coady SA, Heiss G, Sorlie PD, Catellier D, Patsch W. Coronary heart disease prediction from lipoprotein cholesterol levels, triglycerides, lipoprotein(a), apolipoproteins A-I and B, and HDL density subfractions: The Atherosclerosis Risk in Communities (ARIC) Study. Circulation. 2001;104:1108-13.

35. Boekholdt SM, Arsenault BJ, Hovingh GK, Mora S, Pedersen TR, Larosa JC, Welch KM, Amarenco P, Demicco DA, Tonkin AM, et al. Levels and changes of HDL cholesterol and apolipoprotein A-I in relation to risk of cardiovascular events among statin-treated patients: a meta-analysis. Circulation. 2013;128:1504-12.

36. Hovingh GK, Bochem AE, Kastelein JJ. Apolipoprotein A-I mimetic peptides. Curr Opin Lipidol. 2010;21:481-6.

37. Nicholls SJ. Apo a-I modulating therapies. Curr Cardiol Rep. 2011;13:537-43.

38. Singh IM, Shishehbor MH, Ansell BJ. High-density lipoprotein as a therapeutic target: a systematic review. JAMA. 2007;298:786-98.

39. Briel M, Ferreira-Gonzalez I, You JJ, Karanicolas PJ, AkI EA, Wu P, Blechacz B, Bassler D, Wei X, Sharman $A$, et al. Association between change in high density lipoprotein cholesterol and cardiovascular disease morbidity and mortality: systematic review and meta-regression analysis. BMJ. 2009;338:b92.

40. Barter PJ, Caulfield M, Eriksson M, Grundy SM, Kastelein JJ, Komajda M, Lopez-Sendon J, Mosca L, Tardif JC, Waters DD, et al. Effects of torcetrapib in patients at high risk for coronary events. N Engl J Med. 2007;357:2109-22.

41. Therond P. Catabolism of lipoproteins and metabolic syndrome. Curr Opin Clin Nutr Metab Care. 2009;12:366-71.

42. Wang H, Eckel RH. Lipoprotein lipase: from gene to obesity. Am J Physiol Endocrinol Metab. 2009;297:E271-88.

43. Muniyappa R, Quon MJ. Insulin action and insulin resistance in vascular endothelium. Curr Opin Clin Nutr Metab Care. 2007;10:523-30.

44. Devaraj S, Wang-Polagruto J, Polagruto J, Keen CL, Jialal I. High-fat, energy-dense, fast-foodstyle breakfast results in an increase in oxidative stress in metabolic syndrome. Metabolism. 2008;57:867-70.

45. Ghanim H, Abuaysheh S, Sia CL, Korzeniewski K, Chaudhuri A, Fernandez-Real JM, Dandona P. Increase in plasma endotoxin concentrations and the expression of Toll-like receptors and suppressor of cytokine signaling-3 in mononuclear cells after a high-fat, high-carbohydrate meal: implications for insulin resistance. Diabetes Care. 2009;32:2281-7. 
46. Hertog MG, Feskens EJ, Hollman PC, Katan MB, Kromhout D. Dietary antioxidant flavonoids and risk of coronary heart disease: the Zutphen Elderly Study. Lancet. 1993;342:1007-11.

47. Hollenberg NK, Martinez G, McCullough M, Meinking T, Passan D, Preston M, Rivera A, Taplin D, Vicaria-Clement M. Aging, acculturation, salt intake, and hypertension in the Kuna of Panama. Hypertension. 1997;29:171-6.

48. Buijsse B, Feskens EJ, Kok FJ, Kromhout D. Cocoa intake, blood pressure, and cardiovascular mortality: the Zutphen Elderly Study. Arch Intern Med. 2006;166:411-7.

49. Janszky I, Mukamal KJ, Ljung R, Ahnve S, Ahlbom A, Hallqvist J. Chocolate consumption and mortality following a first acute myocardial infarction: the Stockholm Heart Epidemiology Program. J Intern Med. 2009;266:248-57.

50. Manach C, Scalbert A, Morand C, Remesy C, Jimenez L. Polyphenols: food sources and bioavailability. Am J Clin Nutr. 2004;79:727-47.

51. Milder IE, Arts IC, van de Putte B, Venema DP, Hollman PC. Lignan contents of Dutch plant foods: a database including lariciresinol, pinoresinol, secoisolariciresinol and matairesinol. $\mathrm{Br} J$ Nutr. 2005;93:393-402.

52. Tokede OA, Gaziano JM, Djousse L. Effects of cocoa products/dark chocolate on serum lipids: a meta-analysis. Eur J Clin Nutr. 2011;65:879-86.

53. Hooper L, Kay C, Abdelhamid A, Kroon PA, Cohn JS, Rimm EB, Cassidy A. Effects of chocolate, cocoa, and flavan-3-ols on cardiovascular health: a systematic review and meta-analysis of randomized trials. Am J Clin Nutr. 2012;95:740-51.

54. Baba S, Osakabe N, Kato Y, Natsume M, Yasuda A, Kido T, Fukuda K, Muto Y, Kondo K. Continuous intake of polyphenolic compounds containing cocoa powder reduces LDL oxidative susceptibility and has beneficial effects on plasma HDL-cholesterol concentrations in humans. Am J Clin Nutr. 2007;85:709-17.

55. Osakabe N, Baba S, Yasuda A, Iwamoto T, Kamiyama M, Takizawa T, Itakura H, Kondo K. Daily cocoa intake reduces the susceptibility of low-density lipoprotein to oxidation as demonstrated in healthy human volunteers. Free Radic Res. 2001;34:93-9.

56. Yasuda A, Natsume M, Sasaki K, Baba S, Nakamura Y, Kanegae M, Nagaoka S. Cacao procyanidins reduce plasma cholesterol and increase fecal steroid excretion in rats fed a highcholesterol diet. BioFactors. 2008;33:211-23.

57. Voloshyna I, Hussaini SM, Reiss AB. Resveratrol in cholesterol metabolism and atherosclerosis. J Med Food. 2012;15:763-73.

58. Cho IJ, Ahn JY, Kim S, Choi MS, Ha TY. Resveratrol attenuates the expression of HMG-CoA reductase mRNA in hamsters. Biochem Biophys Res Commun. 2008;367:190-4.

59. Berrougui H, Grenier G, Loued S, Drouin G, Khalil A. A new insight into resveratrol as an atheroprotective compound: Inhibition of lipid peroxidation and enhancement of cholesterol efflux. Atherosclerosis. 2009;207:420-7.

60. Wang H, Yang YJ, Qian HY, Zhang Q, Xu H, Li JJ. Resveratrol in cardiovascular disease: what is known from current research? Heart Fail Rev. 2012;17:437-48.

61. Sahebkar A. Effects of resveratrol supplementation on plasma lipids: a systematic review and meta-analysis of randomized controlled trials. Nutr Rev. 2013.

62. Kim A, Chiu A, Barone MK, Avino D, Wang F, Coleman Cl, Phung OJ. Green tea catechins decrease total and low-density lipoprotein cholesterol: a systematic review and meta-analysis. J Am Diet Assoc. 2011;111:1720-9. 
63. Weggemans RM, Trautwein EA. Relation between soy-associated isoflavones and LDL and HDL cholesterol concentrations in humans: a meta-analysis. Eur J Clin Nutr. 2003;57:940-6.

64. Reynolds K, Chin A, Lees KA, Nguyen A, Bujnowski D, He J. A meta-analysis of the effect of soy protein supplementation on serum lipids. The American journal of cardiology. 2006;98:633-40.

65. Zhan S, Ho SC. Meta-analysis of the effects of soy protein containing isoflavones on the lipid profile. Am J Clin Nutr. 2005;81:397-408.

66. Shrime MG, Bauer SR, McDonald AC, Chowdhury NH, Coltart CE, Ding EL. Flavonoid-rich cocoa consumption affects multiple cardiovascular risk factors in a meta-analysis of short-term studies. The Journal of nutrition. 2011;141:1982-8.

67. Kay CD, Hooper L, Kroon PA, Rimm EB, Cassidy A. Relative impact of flavonoid composition, dose and structure on vascular function: a systematic review of randomised controlled trials of flavonoid-rich food products. Mol Nutr Food Res. 2012;56:1605-16.

68. Ramirez-Sanchez I, Maya L, Ceballos G, Villarreal F. (-)-epicatechin activation of endothelial cell endothelial nitric oxide synthase, nitric oxide, and related signaling pathways. Hypertension. 2010;55:1398-405.

69. Gardner EJ, Ruxton CH, Leeds AR. Black tea--helpful or harmful? A review of the evidence. Eur J Clin Nutr. 2007;61:3-18.

70. Kim JA, Montagnani M, Koh KK, Quon MJ. Reciprocal relationships between insulin resistance and endothelial dysfunction: molecular and pathophysiological mechanisms. Circulation. 2006;113:1888-904.

71. Wong RH, Howe PR, Buckley JD, Coates AM, Kunz I, Berry NM. Acute resveratrol supplementation improves flow-mediated dilatation in overweight/obese individuals with mildly elevated blood pressure. Nutr Metab Cardiovasc Dis.

72. Li H, Xia N, Forstermann U. Cardiovascular effects and molecular targets of resveratrol. Nitric oxide : biology and chemistry / official journal of the Nitric Oxide Society. 2012;26:102-10.

73. Bokkenheuser VD, Shackleton $\mathrm{CH}$, Winter J. Hydrolysis of dietary flavonoid glycosides by strains of intestinal Bacteroides from humans. The Biochemical journal. 1987;248:953-6.

74. Hollman PCH. Absorption, bioavailability, and metabolism of flavonoids. Pharm Biol. 2004;42:74-83.

75. Manach C, Williamson G, Morand C, Scalbert A, Remesy C. Bioavailability and bioefficacy of polyphenols in humans. I. Review of 97 bioavailability studies. Am J Clin Nutr. 2005;81:230S-42S.

76. EFSA (European Food Safety Authority). Scientific Opinion on the substantiation of a health claim related to cocoa flavanols and maintenance of normal endothelium-dependent vasodilation pursuant to Article 13(5) of Regulation (EC) No 1924/2006. EFSA Journal. 2012;10:2809.

77. Castelli WP, Garrison RJ, Wilson PW, Abbott RD, Kalousdian S, Kannel WB. Incidence of coronary heart disease and lipoprotein cholesterol levels. The Framingham Study. JAMA. 1986;256:2835-8.

78. Barter PJ, Caulfield M, Eriksson M, Grundy SM, Kastelein JJ, Komajda M, Lopez-Sendon J, Mosca L, Tardif JC, Waters DD, et al. Effects of torcetrapib in patients at high risk for coronary events. N Engl J Med. 2007;357:2109-22.

79. Boden WE, Probstfield JL, Anderson T, Chaitman BR, Desvignes-Nickens P, Koprowicz K, McBride R, Teo K, Weintraub W. Niacin in patients with low HDL cholesterol levels receiving intensive statin therapy. N Engl J Med. 2011;365:2255-67. 
80. Schwartz GG, Olsson AG, Abt M, Ballantyne CM, Barter PJ, Brumm J, Chaitman BR, Holme IM, Kallend D, Leiter LA, et al. Effects of dalcetrapib in patients with a recent acute coronary syndrome. N Engl J Med. 2012;367:2089-99.

81. Manach C, Mazur A, Scalbert A. Polyphenols and prevention of cardiovascular diseases. Curr Opin Lipidol. 2005;16:77-84.

82. Fan E, Zhang L, Jiang S, Bai Y. Beneficial effects of resveratrol on atherosclerosis. J Med Food. 2008;11:610-4.

83. Iannelli P, Zarrilli V, Varricchio E, Tramontano D, Mancini FP. The dietary antioxidant resveratrol affects redox changes of PPARalpha activity. Nutr Metab Cardiovasc Dis. 2007;17:24756.

84. Inoue H, Jiang XF, Katayama T, Osada S, Umesono K, Namura S. Brain protection by resveratrol and fenofibrate against stroke requires peroxisome proliferator-activated receptor alpha in mice. Neurosci Lett. 2003;352:203-6.

85. Erlund I, Koli R, Alfthan G, Marniemi J, Puukka P, Mustonen P, Mattila P, Jula A. Favorable effects of berry consumption on platelet function, blood pressure, and HDL cholesterol. Am J Clin Nutr. 2008;87:323-31.

86. Sacanella E, Vazquez-Agell M, Mena MP, Antunez E, Fernandez-Sola J, Nicolas JM, LamuelaRaventos RM, Ros E, Estruch R. Down-regulation of adhesion molecules and other inflammatory biomarkers after moderate wine consumption in healthy women: a randomized trial. Am J Clin Nutr. 2007;86:1463-9.

87. Poulsen MM, Vestergaard PF, Clasen BF, Radko Y, Christensen LP, Stodkilde-Jorgensen $H$, Moller N, Jessen N, Pedersen SB, Jorgensen JO. High-dose resveratrol supplementation in obese men: an investigator-initiated, randomized, placebo-controlled clinical trial of substrate metabolism, insulin sensitivity, and body composition. Diabetes. 2013;62:1186-95.

88. Timmers S, Konings E, Bilet L, Houtkooper RH, van de Weijer T, Goossens GH, Hoeks J, van der Krieken S, Ryu D, Kersten $S$, et al. Calorie restriction-like effects of 30 days of resveratrol supplementation on energy metabolism and metabolic profile in obese humans. Cell Metab. 2011;14:612-22.

89. Yoshino J, Conte C, Fontana L, Mittendorfer B, Imai S, Schechtman KB, Gu C, Kunz I, Rossi Fanelli F, Patterson BW, et al. Resveratrol supplementation does not improve metabolic function in nonobese women with normal glucose tolerance. Cell Metab. 2012;16:658-64.

90. Friedewald WT, Levy RI, Fredrickson DS. Estimation of the concentration of low-density lipoprotein cholesterol in plasma, without use of the preparative ultracentrifuge. Clin Chem. 1972;18:499-502.

91. Matthews DR, Hosker JP, Rudenski AS, Naylor BA, Treacher DF, Turner RC. Homeostasis model assessment: insulin resistance and beta-cell function from fasting plasma glucose and insulin concentrations in man. Diabetologia. 1985;28:412-9.

92. Hansen HC, Chiacchia FS, Patel R, Wong NC, Khlebnikov V, Jankowska R, Patel K, Reddy MM. Stilbene analogs as inducers of apolipoprotein-I transcription. Eur J Med Chem. 2010;45:2018-23.

93. Dash S, Xiao C, Morgantini C, Szeto L, Lewis GF. High-dose resveratrol treatment for 2 weeks inhibits intestinal and hepatic lipoprotein production in overweight/obese men. Arterioscler Thromb Vasc Biol. 2013;33:2895-901.

94. Brasnyo P, Molnar GA, Mohas M, Marko L, Laczy B, Cseh J, Mikolas E, Szijarto IA, Merei A, Halmai R, et al. Resveratrol improves insulin sensitivity, reduces oxidative stress and activates the Akt pathway in type 2 diabetic patients. Br J Nutr. 2011;106:383-9. 
95. Bhatt JK, Thomas S, Nanjan MJ. Resveratrol supplementation improves glycemic control in type 2 diabetes mellitus. Nutr Res. 2012;32:537-41.

96. Csiszar A, Smith K, Labinskyy N, Orosz Z, Rivera A, Ungvari Z. Resveratrol attenuates TNFalpha-induced activation of coronary arterial endothelial cells: role of NF-kappaB inhibition. Am J Physiol Heart Circ Physiol. 2006;291:H1694-9.

97. Agarwal B, Campen MJ, Channell MM, Wherry SJ, Varamini B, Davis JG, Baur JA, Smoliga JM. Resveratrol for primary prevention of atherosclerosis: clinical trial evidence for improved gene expression in vascular endothelium. Int J Cardiol. 2013;166:246-8.

98. Wong RH, Berry NM, Coates AM, Buckley JD, Bryan J, Kunz I, Howe PR. Chronic resveratrol consumption improves brachial flow-mediated dilatation in healthy obese adults. J Hypertens. 2013;31:1819-27.

99. Bode LM, Bunzel D, Huch M, Cho GS, Ruhland D, Bunzel M, Bub A, Franz CM, Kulling SE. In vivo and in vitro metabolism of trans-resveratrol by human gut microbiota. Am J Clin Nutr. 2013;97:295-309.

100. Baur JA, Sinclair DA. Therapeutic potential of resveratrol: the in vivo evidence. Nat Rev Drug Discov. 2006;5:493-506.

101. Vang O, Ahmad N, Baile CA, Baur JA, Brown K, Csiszar A, Das DK, Delmas D, Gottfried C, Lin HY, et al. What is new for an old molecule? Systematic review and recommendations on the use of resveratrol. PLoS One. 2011;6:e19881.

102. van der Made SM, Plat J, Mensink RP. Resveratrol does not influence metabolic risk markers related to cardiovascular health in overweight and slightly obese subjects: a randomized, placebocontrolled crossover trial. Accepted for publication.

103. Wallerath T, Deckert G, Ternes T, Anderson H, Li H, Witte K, Forstermann U. Resveratrol, a polyphenolic phytoalexin present in red wine, enhances expression and activity of endothelial nitric oxide synthase. Circulation. 2002;106:1652-8.

104. Takizawa Y, Kosuge Y, Awaji H, Tamura E, Takai A, Yanai T, Yamamoto R, Kokame K, Miyata T, Nakata $R$, et al. Up-regulation of endothelial nitric oxide synthase (eNOS), silent mating type information regulation 2 homologue 1 (SIRT1) and autophagy-related genes by repeated treatments with resveratrol in human umbilical vein endothelial cells. Br J Nutr. 2013;110:2150-5.

105. Wong RH, Howe PR, Buckley JD, Coates AM, Kunz I, Berry NM. Acute resveratrol supplementation improves flow-mediated dilatation in overweight/obese individuals with mildly elevated blood pressure. Nutr Metab Cardiovasc Dis. 2011;21:851-6.

106. Magyar K, Halmosi R, Palfi A, Feher G, Czopf L, Fulop A, Battyany I, Sumegi B, Toth K, Szabados E. Cardioprotection by resveratrol: A human clinical trial in patients with stable coronary artery disease. Clin Hemorheol Microcirc. 2012;50:179-87.

107. Gaenzer H, Sturm W, Neumayr G, Kirchmair R, Ebenbichler C, Ritsch A, Foger B, Weiss G, Patsch JR. Pronounced postprandial lipemia impairs endothelium-dependent dilation of the brachial artery in men. Cardiovasc Res. 2001;52:509-16.

108. Vogel RA, Corretti MC, Plotnick GD. Effect of a single high-fat meal on endothelial function in healthy subjects. Am J Cardiol. 1997;79:350-4.

109. Matthews JN, Altman DG, Campbell MJ, Royston P. Analysis of serial measurements in medical research. BMJ. 1990;300:230-5. 
110. Bradamante S, Barenghi L, Piccinini F, Bertelli AA, De Jonge R, Beemster $P$, De Jong JW. Resveratrol provides late-phase cardioprotection by means of a nitric oxide- and adenosinemediated mechanism. Eur J Pharmacol. 2003;465:115-23.

111. Zhang H, Zhang J, Ungvari Z, Zhang C. Resveratrol Improves Endothelial Function. Role of TNF $\{a l p h a\}$ and Vascular Oxidative Stress. Arterioscler Thromb Vasc Biol. 2009.

112. Rotches-Ribalta M, Andres-Lacueva C, Estruch R, Escribano E, Urpi-Sarda M. Pharmacokinetics of resveratrol metabolic profile in healthy humans after moderate consumption of red wine and grape extract tablets. Pharmacol Res. 2012;66:375-82.

113. Bae JH, Schwemmer M, Lee IK, Lee HJ, Park KR, Kim KY, Bassenge E. Postprandial hypertriglyceridemia-induced endothelial dysfunction in healthy subjects is independent of lipid oxidation. Int J Cardiol. 2003;87:259-67.

114. Joris PJ, Mensink RP. Beetroot juice improves in overweight and slightly obese men postprandial endothelial function after consumption of a mixed meal. Atherosclerosis. 2013;231:78-83.

115. Johnson BD, Padilla J, Harris RA, Wallace JP. Vascular consequences of a high-fat meal in physically active and inactive adults. Appl Physiol Nutr Metab. 2011;36:368-75.

116. Westphal S, Luley C. Flavanol-rich cocoa ameliorates lipemia-induced endothelial dysfunction. Heart Vessels. 2011;26:511-5.

117. Lynn A, Hamadeh H, Leung WC, Russell JM, Barker ME. Effects of pomegranate juice supplementation on pulse wave velocity and blood pressure in healthy young and middle-aged men and women. Plant Foods Hum Nutr. 2012;67:309-14.

118. West SG, Mclntyre MD, Piotrowski MJ, Poupin N, Miller DL, Preston AG, Wagner P, Groves LF, Skulas-Ray AC. Effects of dark chocolate and cocoa consumption on endothelial function and arterial stiffness in overweight adults. Br J Nutr. 2014;111:653-61.

119. Wung BS, Hsu MC, Wu CC, Hsieh CW. Resveratrol suppresses IL-6-induced ICAM-1 gene expression in endothelial cells: effects on the inhibition of STAT3 phosphorylation. Life Sci. 2005;78:389-97.

120. Ferrero ME, Bertelli AE, Fulgenzi A, Pellegatta F, Corsi MM, Bonfrate M, Ferrara F, De Caterina $R$, Giovannini L, Bertelli A. Activity in vitro of resveratrol on granulocyte and monocyte adhesion to endothelium. Am J Clin Nutr. 1998;68:1208-14.

121. Ghanim H, Sia CL, Korzeniewski K, Lohano T, Abuaysheh S, Marumganti A, Chaudhuri A, Dandona P. A resveratrol and polyphenol preparation suppresses oxidative and inflammatory stress response to a high-fat, high-carbohydrate meal. J Clin Endocrinol Metab. 2011;96:1409-14.

122. Tome-Carneiro J, Gonzalvez M, Larrosa M, Yanez-Gascon MJ, Garcia-Almagro FJ, Ruiz-Ros JA, Garcia-Conesa MT, Tomas-Barberan FA, Espin JC. One-year consumption of a grape nutraceutical containing resveratrol improves the inflammatory and fibrinolytic status of patients in primary prevention of cardiovascular disease. Am J Cardiol. 2012;110:356-63.

123. Applegate E. Introduction: nutritional and functional roles of eggs in the diet. J Am Coll Nutr. 2000;19:495S-8S.

124. Lichtenstein AH, Appel LJ, Brands M, Carnethon M, Daniels S, Franch HA, Franklin B, KrisEtherton P, Harris WS, Howard B, et al. Diet and lifestyle recommendations revision 2006: a scientific statement from the American Heart Association Nutrition Committee. Circulation. 2006;114:82-96.

125. U.S. Department of Agriculture and U.S. Department of Health and Human Services. Dietary Guidelines for Americans, 2010. 7th ed. Washington, DC: Government Printing Office. 
126. Weggemans RM, Zock PL, Katan MB. Dietary cholesterol from eggs increases the ratio of total cholesterol to high-density lipoprotein cholesterol in humans: a meta-analysis. Am J Clin Nutr. 2001;73:885-91.

127. Silbernagel G, Fauler G, Hoffmann MM, Lutjohann D, Winkelmann BR, Boehm BO, Marz W. The associations of cholesterol metabolism and plasma plant sterols with all-cause and cardiovascular mortality. J Lipid Res. 2010;51:2384-93.

128. Herron KL, Lofgren IE, Sharman M, Volek JS, Fernandez ML. High intake of cholesterol results in less atherogenic low-density lipoprotein particles in men and women independent of response classification. Metabolism. 2004;53:823-30.

129. Rong Y, Chen L, Zhu T, Song Y, Yu M, Shan Z, Sands A, Hu FB, Liu L. Egg consumption and risk of coronary heart disease and stroke: dose-response meta-analysis of prospective cohort studies. BMJ. 2013;346:e8539.

130. Spence JD, Jenkins DJ, Davignon J. Egg yolk consumption and carotid plaque. Atherosclerosis. 2012;224:469-73.

131. Kromhout D, Geleijnse JM, Menotti A, Jacobs DR, Jr. The confusion about dietary fatty acids recommendations for CHD prevention. Br J Nutr. 2011;106:627-32.

132. Westerterp KR, Goris AH. Validity of the assessment of dietary intake: problems of misreporting. Curr Opin Clin Nutr Metab Care. 2002;5:489-93.

133. Chung HY, Rasmussen HM, Johnson EJ. Lutein bioavailability is higher from lutein-enriched eggs than from supplements and spinach in men. J Nutr. 2004;134:1887-93.

134. Maiani G, Caston MJ, Catasta G, Toti E, Cambrodon IG, Bysted A, Granado-Lorencio F, Olmedilla-Alonso B, Knuthsen P, Valoti M, et al. Carotenoids: actual knowledge on food sources, intakes, stability and bioavailability and their protective role in humans. Mol Nutr Food Res. 2009;53 Suppl 2:S194-218.

135. Wang W, Connor SL, Johnson EJ, Klein ML, Hughes S, Connor WE. Effect of dietary lutein and zeaxanthin on plasma carotenoids and their transport in lipoproteins in age-related macular degeneration. Am J Clin Nutr. 2007;85:762-9.

136. Kijlstra A, Tian Y, Kelly ER, Berendschot TT. Lutein: More than just a filter for blue light. Prog Retin Eye Res. 2012;31:303-15.

137. Kim SR, Nakanishi K, Itagaki Y, Sparrow JR. Photooxidation of A2-PE, a photoreceptor outer segment fluorophore, and protection by lutein and zeaxanthin. Exp Eye Res. 2006;82:828-39.

138. Neuringer M, Sandstrom MM, Johnson EJ, Snodderly DM. Nutritional manipulation of primate retinas, I: effects of lutein or zeaxanthin supplements on serum and macular pigment in xanthophyll-free rhesus monkeys. Invest Ophthalmol Vis Sci. 2004;45:3234-43.

139. Prokofyeva E, Zrenner E. Epidemiology of major eye diseases leading to blindness in Europe: a literature review. Ophthalmic Res. 2012;47:171-88.

140. Sabour-Pickett S, Nolan JM, Loughman J, Beatty S. A review of the evidence germane to the putative protective role of the macular carotenoids for age-related macular degeneration. Mol Nutr Food Res. 2012;56:270-86.

141. Bosner MS, Ostlund RE, Jr., Osofisan O, Grosklos J, Fritschle C, Lange LG. Assessment of percent cholesterol absorption in humans with stable isotopes. J Lipid Res. 1993;34:1047-53. 
142. Miettinen TA, Gylling H, Strandberg T, Sarna S. Baseline serum cholestanol as predictor of recurrent coronary events in subgroup of Scandinavian simvastatin survival study. Finnish $4 \mathrm{~S}$ Investigators. BMJ. 1998;316:1127-30.

143. Thuluva SC, Igel M, Giesa U, Lutjohann D, Sudhop T, von Bergmann K. Ratio of lathosterol to campesterol in serum predicts the cholesterol-lowering effect of sitostanol-supplemented margarine. Int J Clin Pharmacol Ther. 2005;43:305-10.

144. Kesaniemi YA, Ehnholm C, Miettinen TA. Intestinal cholesterol absorption efficiency in man is related to apoprotein E phenotype. J Clin Invest. 1987;80:578-81.

145. Miettinen TA, Gylling H, Vanhanen H. Serum cholesterol response to dietary cholesterol and apoprotein E phenotype. Lancet. 1988;2:1261.

146. McKay GJ, Patterson CC, Chakravarthy U, Dasari S, Klaver CC, Vingerling JR, Ho L, de Jong PT, Fletcher AE, Young IS, et al. Evidence of association of APOE with age-related macular degeneration: a pooled analysis of 15 studies. Hum Mutat. 2011;32:1407-16.

147. Plat J, Mensink RP. Effects of diets enriched with two different plant stanol ester mixtures on plasma ubiquinol-10 and fat-soluble antioxidant concentrations. Metabolism. 2001;50:520-9.

148. Mensink RP, de Jong A, Lutjohann D, Haenen GR, Plat J. Plant stanols dose-dependently decrease LDL-cholesterol concentrations, but not cholesterol-standardized fat-soluble antioxidant concentrations, at intakes up to 9 g/d. Am J Clin Nutr. 2010;92:24-33.

149. Minihane AM, Jofre-Monseny L, Olano-Martin E, Rimbach G. ApoE genotype, cardiovascular risk and responsiveness to dietary fat manipulation. P Nutr Soc. 2007;66:183-97.

150. Tan JS, Wang JJ, Flood V, Rochtchina E, Smith W, Mitchell P. Dietary antioxidants and the longterm incidence of age-related macular degeneration: the Blue Mountains Eye Study. Ophthalmology. 2008;115:334-41.

151. The Age-Related Eye Disease Study 2 Research Group, Chew EY, Clemons TE, Sangiovanni JP, Danis RP, Ferris FL, Elman MJ, Antoszyk AN, Ruby AJ, Orth D, et al. Secondary Analyses of the Effects of Lutein/Zeaxanthin on Age-Related Macular Degeneration Progression: AREDS2 Report No. 3. JAMA Ophthalmol. 2013.

152. Berendschot TT, Goldbohm RA, Klopping WA, van de Kraats J, van Norel J, van Norren D. Influence of lutein supplementation on macular pigment, assessed with two objective techniques. Invest Ophthalmol Vis Sci. 2000;41:3322-6.

153. Huang YM, Yan SF, Ma L, Zou ZY, Xu XR, Dou HL, Lin XM. Serum and macular responses to multiple xanthophyll supplements in patients with early age-related macular degeneration. Nutrition. 2013;29:387-92.

154. Handelman GJ, Nightingale ZD, Lichtenstein AH, Schaefer EJ, Blumberg JB. Lutein and zeaxanthin concentrations in plasma after dietary supplementation with egg yolk. Am J Clin Nutr. 1999;70:247-51.

155. Baigent C, Keech A, Kearney PM, Blackwell L, Buck G, Pollicino C, Kirby A, Sourjina T, Peto R, Collins R, et al. Efficacy and safety of cholesterol-lowering treatment: prospective meta-analysis of data from 90,056 participants in 14 randomised trials of statins. Lancet. 2005;366:1267-78.

156. Cholesterol Treatment Trialists Collaboration, Baigent C, Blackwell L, Emberson J, Holland LE, Reith C, Bhala N, Peto R, Barnes EH, Keech A, et al. Efficacy and safety of more intensive lowering of LDL cholesterol: a meta-analysis of data from 170,000 participants in 26 randomised trials. Lancet. 2010;376:1670-81.

157. Arsenault BJ, Rana JS, Stroes ES, Despres JP, Shah PK, Kastelein JJ, Wareham NJ, Boekholdt SM, Khaw KT. Beyond low-density lipoprotein cholesterol: respective contributions of non-high- 
density lipoprotein cholesterol levels, triglycerides, and the total cholesterol/high-density lipoprotein cholesterol ratio to coronary heart disease risk in apparently healthy men and women. J Am Coll Cardiol. 2009;55:35-41.

158. Blanco-Molina A, Castro G, Martin-Escalante D, Bravo D, Lopez-Miranda J, Castro P, LopezSegura F, Fruchart JC, Ordovas JM, Perez-Jimenez F. Effects of different dietary cholesterol concentrations on lipoprotein plasma concentrations and on cholesterol efflux from Fu5AH cells. Am J Clin Nutr. 1998;68:1028-33.

159. Ginsberg HN, Karmally W, Siddiqui M, Holleran S, Tall AR, Blaner WS, Ramakrishnan R. Increases in dietary cholesterol are associated with modest increases in both LDL and HDL cholesterol in healthy young women. Arterioscler Thromb Vasc Biol. 1995;15:169-78.

160. Ginsberg HN, Karmally W, Siddiqui M, Holleran S, Tall AR, Rumsey SC, Deckelbaum RJ, Blaner WS, Ramakrishnan R. A dose-response study of the effects of dietary cholesterol on fasting and postprandial lipid and lipoprotein metabolism in healthy young men. Arteriosclerosis and thrombosis : a journal of vascular biology / American Heart Association. 1994;14:576-86.

161. Herron KL, Vega-Lopez S, Conde K, Ramjiganesh T, Roy S, Shachter NS, Fernandez ML. Premenopausal women, classified as hypo- or hyperresponders, do not alter their LDL/HDL ratio following a high dietary cholesterol challenge. J Am Coll Nutr. 2002;21:250-8.

162. Sacks FM, Salazar J, Miller L, Foster JM, Sutherland M, Samonds KW, Albers JJ, Kass EH. Ingestion of egg raises plasma low density lipoproteins in free-living subjects. Lancet. 1984;1:6479.

163. Baumgartner S, Kelly ER, van der Made S, Berendschot TT, Husche C, Lutjohann D, Plat J. The influence of consuming an egg or an egg-yolk buttermilk drink for 12 wk on serum lipids, inflammation, and liver function markers in human volunteers. Nutrition. 2013.

164. Duane WC. Serum lathosterol levels in human subjects reflect changes in whole body cholesterol synthesis induced by lovastatin but not dietary cholesterol. J Lipid Res. 1995;36:343-8.

165. Zou Z, Xu X, Huang Y, Xiao X, Ma L, Sun T, Dong P, Wang X, Lin X. High serum level of lutein may be protective against early atherosclerosis: the Beijing atherosclerosis study. Atherosclerosis. 2011;219:789-93.

166. Plat J, Mensink RP. Relationship of genetic variation in genes encoding apolipoprotein A-IV, scavenger receptor $\mathrm{BI}, \mathrm{HMG}-\mathrm{COA}$ reductase, CETP and apolipoprotein $\mathrm{E}$ with cholesterol metabolism and the response to plant stanol ester consumption. Eur J Clin Invest. 2002;32:242-50.

167. Lovegrove JA, Griffin BA. The acute and long-term effects of dietary fatty acids on vascular function in health and disease. Curr Opin Clin Nutr Metab Care. 2013;16:162-7.

168. Goldberg S, Gardener H, Tiozzo E, Ying Kuen C, Elkind MS, Sacco RL, Rundek T. Egg consumption and carotid atherosclerosis in the Northern Manhattan Study. Atherosclerosis. 2014;235:273-80.

169. Wang L, Gaziano JM, Norkus EP, Buring JE, Sesso HD. Associations of plasma carotenoids with risk factors and biomarkers related to cardiovascular disease in middle-aged and older women. Am J Clin Nutr. 2008;88:747-54.

170. Ford ES, Mokdad AH, Giles WH, Brown DW. The metabolic syndrome and antioxidant concentrations: findings from the Third National Health and Nutrition Examination Survey. Diabetes. 2003;52:2346-52. 
171. Watarai R, Suzuki K, Ichino N, Osakabe K, Sugimoto K, Yamada H, Hamajima T, Hamajima N, Inoue $T$. Association between serum levels of carotenoids and serum asymmetric dimethylarginine levels in Japanese subjects. J Epidemiol. 2014;24:250-7.

172. Zou ZY, Xu XR, Lin XM, Zhang HB, Xiao X, Ouyang L, Huang YM, Wang X, Liu YQ. Effects of lutein and lycopene on carotid intima-media thickness in Chinese subjects with subclinical atherosclerosis: a randomised, double-blind, placebo-controlled trial. Br J Nutr. 2014;111:474-80.

173. van der Made SM, Kelly ER, Berendschot TT, Kijlstra A, Lutjohann D, Plat J. Consuming a Buttermilk Drink Containing Lutein-Enriched Egg Yolk Daily for 1 Year Increased Plasma Lutein but Did Not Affect Serum Lipid or Lipoprotein Concentrations in Adults with Early Signs of Age-Related Macular Degeneration. J Nutr. 2014.

174. Laplante MA, Charbonneau A, Avramoglu RK, Pelletier P, Fang X, Bachelard H, Yla-Herttuala S, Laakso M, Despres JP, Deshaies $Y$, et al. Distinct metabolic and vascular effects of dietary triglycerides and cholesterol in atherosclerotic and diabetic mouse models. Am J Physiol Endocrinol Metab. 2013;305:E573-84.

175. Cholesterol Treatment Trialists C, Baigent C, Blackwell L, Emberson J, Holland LE, Reith C, Bhala N, Peto R, Barnes EH, Keech A, et al. Efficacy and safety of more intensive lowering of LDL cholesterol: a meta-analysis of data from 170,000 participants in 26 randomised trials. Lancet. 2010;376:1670-81.

176. Hu FB, Stampfer MJ, Rimm EB, Manson JE, Ascherio A, Colditz GA, Rosner BA, Spiegelman D, Speizer FE, Sacks FM, et al. A prospective study of egg consumption and risk of cardiovascular disease in men and women. JAMA. 1999;281:1387-94.

177. Zazpe I, Beunza JJ, Bes-Rastrollo M, Warnberg J, de la Fuente-Arrillaga C, Benito S, Vazquez Z, Martinez-Gonzalez MA, Investigators SUNP. Egg consumption and risk of cardiovascular disease in the SUN Project. Eur J Clin Nutr. 2011;65:676-82.

178. Deanfield JE, Halcox JP, Rabelink TJ. Endothelial function and dysfunction: testing and clinical relevance. Circulation. 2007;115:1285-95.

179. Katz DL, Evans MA, Nawaz H, Njike VY, Chan W, Comerford BP, Hoxley ML. Egg consumption and endothelial function: a randomized controlled crossover trial. Int J Cardiol. 2005;99:65-70.

180. Njike V, Faridi Z, Dutta S, Gonzalez-Simon AL, Katz DL. Daily egg consumption in hyperlipidemic adults--effects on endothelial function and cardiovascular risk. Nutr J. 2010;9:28.

181. Armoza A, Haim Y, Bashiri A, Wolak T, Paran E. Tomato extract and the carotenoids lycopene and lutein improve endothelial function and attenuate inflammatory NF-kappaB signaling in endothelial cells. J Hypertens. 2013;31:521-9; discussion 9.

182. van Herpen-Broekmans WM, Klopping-Ketelaars IA, Bots ML, Kluft C, Princen H, Hendriks HF, Tijburg LB, van Poppel G, Kardinaal AF. Serum carotenoids and vitamins in relation to markers of endothelial function and inflammation. Eur J Epidemiol. 2004;19:915-21.

183. Herron KL, Lofgren IE, Sharman M, Volek JS, Fernandez ML. High intake of cholesterol results in less atherogenic low-density lipoprotein particles in men and women independent of response classification. Metabolism. 2004;53:823-30.

184. Okumura K, Takahashi R, Taguchi N, Suzuki M, Cheng XW, Numaguchi Y, Ikeda N, Murohara T. Small low-density lipoprotein cholesterol concentration is a determinant of endothelial dysfunction by peripheral artery tonometry in men. J Atheroscler Thromb. 2012;19:897-903.

185. Vakkilainen J, Makimattila S, Seppala-Lindroos A, Vehkavaara S, Lahdenpera S, Groop PH, Taskinen MR, Yki-Jarvinen $\mathrm{H}$. Endothelial dysfunction in men with small LDL particles. Circulation. 2000;102:716-21. 
186. Wakatsuki A, Ikenoue N, Shinohara K, Watanabe K, Fukaya T. Small low-density lipoprotein particles and endothelium-dependent vasodilation in postmenopausal women. Atherosclerosis. 2004;177:329-36

187. Andersen CJ, Fernandez ML. Dietary strategies to reduce metabolic syndrome. Rev Endocr Metab Disord. 2013;14:241-54.

188. Konings E, Timmers S, Boekschoten MV, Goossens GH, Jocken JW, Afman LA, Muller M, Schrauwen P, Mariman EC, Blaak EE. The effects of 30 days resveratrol supplementation on adipose tissue morphology and gene expression patterns in obese men. Int J Obes (Lond). 2014;38:470-3.

189. Investigators A-H, Boden WE, Probstfield JL, Anderson T, Chaitman BR, Desvignes-Nickens P, Koprowicz K, McBride R, Teo K, Weintraub W. Niacin in patients with low HDL cholesterol levels receiving intensive statin therapy. N Engl J Med. 2011;365:2255-67.

190. Rohatgi A, Khera A, Berry JD, Givens EG, Ayers CR, Wedin KE, Neeland IJ, Yuhanna IS, Rader DR, de Lemos JA, et al. HDL Cholesterol Efflux Capacity and Incident Cardiovascular Events. N Engl J Med. 2014.

191. Soran H, Hama S, Yadav R, Durrington PN. HDL functionality. Curr Opin Lipidol. 2012;23:35366.

192. Voloshyna I, Hai O, Littlefield MJ, Carsons S, Reiss AB. Resveratrol mediates anti-atherogenic effects on cholesterol flux in human macrophages and endothelium via PPARgamma and adenosine. Eur J Pharmacol. 2013;698:299-309.

193. Allen AM, Graham A. Mitochondrial function is involved in regulation of cholesterol efflux to apolipoprotein (apo)A-I from murine RAW 264.7 macrophages. Lipids Health Dis. 2012;11:169.

194. Prokofyeva E, Zrenner E. Epidemiology of major eye diseases leading to blindness in Europe: a literature review. Ophthalmic Res. 2012;47:171-88.

195. Cotterill OJ, Marion WW, Naber EC. A nutrient re-evaluation of shell eggs. Poult Sci. 1977;56:1927-34.

196. Kaput J. Nutrigenomics research for personalized nutrition and medicine. Curr Opin Biotechnol. 2008;19:110-20.

197. Loane E, Nolan JM, Beatty S. The respective relationships between lipoprotein profile, macular pigment optical density, and serum concentrations of lutein and zeaxanthin. Invest Ophthalmol Vis Sci.51:5897-905.

198. Ras RT, Streppel MT, Draijer R, Zock PL. Flow-mediated dilation and cardiovascular risk prediction: a systematic review with meta-analysis. Int J Cardiol. 2013;168:344-51.

199. Ma L, Yan SF, Huang YM, Lu XR, Qian F, Pang HL, Xu XR, Zou ZY, Dong PC, Xiao X, et al. Effect of lutein and zeaxanthin on macular pigment and visual function in patients with early age-related macular degeneration. Ophthalmology. 2012;119:2290-7.

200. WHO. Constitution of the World Health Organization. Basic Documents. 2006.

201. Huber M, Knottnerus JA, Green L, van der Horst H, Jadad AR, Kromhout D, Leonard B, Lorig K, Loureiro MI, van der Meer JW, et al. How should we define health? BMJ. 2011;343:d4163.

202. van Drooge L, Vandeberg R, Zuijdam F, Mostert B, van der Meulen B, Bruins E. Valuable: Indicators for Valorisation. The Hague: Rathenau Institute; 2013.

203. WHO. The European health report 2012: charting the way to well-being. Copenhagen: World Health Organization Regional Office for Europe; 2014. 
204. Wong WL, Su X, Li X, Cheung CM, Klein R, Cheng CY, Wong TY. Global prevalence of agerelated macular degeneration and disease burden projection for 2020 and 2040: a systematic review and meta-analysis. Lancet Glob Health. 2014;2:e106-16. 

Summary 
The metabolic syndrome (MetS) is a complex of metabolic risk markers for cardiovascular disease (CVD) and type 2 diabetes mellitus (T2DM). These risk markers include an elevated waist circumference, atherogenic dyslipidemia -as evidenced by an increased fasting serum concentration of triacylglycerols and low HDL cholesterol concentrations-, raised blood pressure and dysglycemia. Several other characteristics are associated with the MetS, such as vascular dysfunction and vascular inflammation. A healthy lifestyle consisting of a healthy diet and sufficient physical exercise is associated with a lower incidence of the MetS and improvement in occurrence of related characteristics. Next to this, the use of functional foods and bioactive ingredients that interfere with typical characteristics of the MetS is a widely used approach to lower the risk for CVD. The aim of the research presented in this thesis is to assess the effects of two nutritional compounds or bioactive ingredients on markers for cardiovascular health such as endothelial function, lipid- and lipoprotein metabolism and glucose metabolism. The relation between the two nutritional compounds and the risk for markers related to the MetS and CVD were assessed in both the fasting and postprandial state.

Chapter 2 gives an overview of the literature regarding the effect of polyphenols, a group of metabolites produced by higher plants on cardiovascular risk markers. We particularly focused on flavonoids from cocoa, the stilbene trans-resveratrol and green tea catechins. Beneficial effects of flavanol-rich cocoa products or dark chocolate consumption on LDL cholesterol concentrations were found in short-term studies $(<2$ weeks), whereas these effects were not confirmed in longer-term studies. In addition, a positive effect of cocoa and cocoa-flavanols on flow-mediated dilation was found. Furthermore, green tea catechins improved the serum lipid profile through decreasing total and LDL cholesterol concentrations, whereas no evidence was found for an effect of trans-resveratrol on serum lipid and lipoprotein concentration. However, evidence was found for a positive effect of trans-resveratrol on vascular health. Therefore, it was decided to evaluate in more detail the effects of trans-resveratrol in a well-controlled dietary intervention study (chapter 3 and 4). Forty-five overweight and slightly obese men and women with low HDL cholesterol concentrations participated in a study that evaluated the effect of daily intake of $150 \mathrm{mg}$ trans-resveratrol for 4-weeks on fasting metabolic risk markers, such as lipid, lipoprotein and glucose concentrations, and markers for inflammation and endothelial function. Capsule count and changes in resveratrol and dihydroresveratrol concentrations during the resveratrol-period indicated excellent compliance. However, no difference between resveratrol and placebo was found in apoA-I, apoB100, HDL cholesterol, LDL cholesterol, total cholesterol, triacyglycerol, glucose and insulin concentrations. Furthermore, we did not find effects on plasma markers for inflammation and endothelial function in the fasting state. Besides the effect of trans-resveratrol intake on fasting markers for metabolic 
health, we also assessed its effect on endothelial function through measuring flowmediated vasodilation, and arterial stiffness by pulse-wave velocity both fasted and in the postprandial phase. FMD and PWV did not change after the resveratrol period. Biomarkers for endothelial function and inflammation were measured during the postprandial test, and responses were not different between the resveratrol and placebo period. To conclude, $150 \mathrm{mg}$ of daily trans-resveratrol intake for 4 weeks did not affect metabolic risk markers related to cardiovascular health both in the fasted state and in the postprandial phase in overweight and slightly obese men and women.

Chapters 5 and 6 describe the effects of consuming a buttermilk drink containing lutein-enriched egg-yolk for one year on cardiovascular health parameters. One hundred and one men and women characterized by early signs of age-related macular degeneration (AMD) participated in this study, as the primary objective was to assess the effects of consuming the lutein-enriched egg drink on plasma lutein concentrations and visual function. The secondary aim of this study was to evaluate the effects of long-term daily egg yolk consumption on cardiovascular risk factors. The increase in plasma lutein concentrations was $83 \%$ greater in the group that consumed the lutein-enriched egg yolk drink compared to the control group. However, changes in serum total, HDL and LDL cholesterol concentrations were not significantly different between the groups. Subgroup analyses showed that subjects classified as 'cholesterol absorber' by serum campesterol:lathosterol ratio might particularly benefit from the lutein-enriched buttermilk drink, as serum HDL cholesterol and plasma lutein concentrations in this group were higher compared to the mid- or low-ration groups. Besides this, one-year consumption of the lutein-enriched egg yolk incorporated in a dairy drink had no effect on postprandial glucose and lipid metabolism or endothelial function as measured by FMD.

Taken together, the studies described in this thesis were designed to assess the effect of dietary interventions on cardiovascular risk markers. Trans-resveratrol was hypothesized to have a positive effect on these markers, whereas a lutein-enriched dairy drink was thought to negatively influence cardiovascular risk markers through increased dietary cholesterol intake. However, no evidence was found for a positive effect of transresveratrol on metabolic risk markers, and no effect was observed after one-year consumption of a lutein-enriched egg yolk buttermilk drink on these markers. 



\section{Samenvatting}


Het metabool syndroom (MetS) is een verzameling van metabole risicofactoren, die het risico op het krijgen van hart- en vaatziekten (HVZ) en type 2 diabetes mellitus (T2DM) verhogen. Risicofactoren die tot het MetS behoren zijn een verhoogde taille omvang, atherogene dyslipidemie -hieronder worden hoge gevaste serum triglyceriden en lage serum HDL cholesterol concentraties verstaan-, een verhoogde bloeddruk en een verstoorde plasma glucosespiegel. Verscheidene andere karakteristieken zijn geassocieerd met het MetS, zoals een verminderde vaatwandfunctie en laaggradige ontsteking. Een gezonde leefstijl, bestaande uit een gezonde voeding en voldoende lichamelijke beweging, gaat samen met een lagere incidentie van het MetS. De inname van functionele voedingsmiddelen of bioactieve stoffen die een gunstig effect hebben op de typische karakteristieken van het MetS, is een veelgebruikte aanpak om het risico op HVZ te verlagen. De doelstelling van het onderzoek, zoals beschreven in deze thesis, is om de effecten te bepalen van een bioactieve stof en een functioneel voedingsmiddel op markers voor cardiovasculaire gezondheid, waaronder vaatwandfunctie, en de vetstof- en glucosestofwisseling. De relatie tussen de bioactieve stof en het functioneel voedingsmiddel op risicomarkers voor het MetS en HVZ zijn zowel in de gevaste als in de postprandiale ("na de maaltijd") staat bekeken.

Hoofdstuk 2 geeft een overzicht van de literatuur waarin effecten van polyfenolen, een groep stoffen afkomstig uit bepaalde planten, op risicomarkers voor cardiovasculaire aandoeningen, worden besproken. In dit hoofdstuk ligt de focus op flavonoïden uit cacao, trans-resveratrol, en catechinen uit groene thee. Korte termijn studies lieten gunstige effecten van flavanolrijke cacao producten of consumptie van donkere chocolade op LDL cholesterol concentraties zien (duur $<2$ weken). Deze resultaten werden echter niet gevonden in langere termijn studies. Verder werd een positief effect van cacao en flavanolen uit cacao op flow-gemedieerde vasodilatatie (FMD) gevonden. De FMD is een maat voor de vaatwandfunctie. Ook verbeterden catechinen uit groene thee het serum lipidenprofiel door het verlagen van totaal- en LDL cholesterol concentraties. Er waren geen aanwijzingen voor een effect van transresveratrol op de serum lipiden- en lipoproteïnenconcentraties. Wel werden aanwijzingen gevonden voor een gunstig effect van trans-resveratrol op vaatwandfunctie. Dit deed ons besluiten om effecten van trans-resveratrol op parameters voor cardiovasculaire gezondheid in meer detail te bestuderen in een gecontroleerde voedingsinterventie studie (hoofdstuk 3 en 4). Vijfenveertig mannen en vrouwen met overgewicht of matige obesitas en lage serum HDL cholesterol concentraties participeerden in deze studie. Hierin werden de effecten van een dagelijkse inname van 150 mg trans-resveratrol, gedurende vier weken, op gevaste 
metabole risicomarkers, zoals lipiden-, lipoproteïnen- en glucoseconcentraties en markers voor laaggradige ontsteking en vaatwandfunctie onderzocht. Het tellen van capsules en de veranderingen in plasma resveratrol en dihydroresveratrolconcentraties lieten zien dat de deelnemers zich zeer goed aan het protocol hadden gehouden. Toch werden geen effecten gevonden van trans-resveratrol op serum concentraties van apoAI, apoB100, HDL-, LDL- en totaal cholesterol, triglyceriden en van plasma glucose en insuline concentraties. Verder werden in de gevaste staat geen effecten gevonden op plasma onstekingsmarkers en markers voor vaatwandfunctie. Naast het effect van transresveratrol op gevaste markers voor metabole gezondheid, is ook het effect op vaatwandfunctie gemeten door de FMD en arteriële stijfheid te bepalen. Arteriële stijfheid werd bepaald door het meten van de snelheid waarmee een drukgolf door de arteriën reist (pulse wave velocity, PWV). Zowel FMD als PWV werden in de gevaste en in de postprandiale staat gemeten, maar veranderden niet door het gebruik van transresveratrol. Ook werden markers voor vaatwandfunctie en laaggradige ontsteking gemeten tijdens de postprandiaaltest. Trans-resveratrol had geen effecten op deze postprandiale responsen. Concluderend kan worden gesteld dat de dagelijkse inname van 150 mg trans-resveratrol voor vier weken geen effect heeft op metabole risicomarkers gerelateerd aan cardiovasculaire gezondheid in mannen en vrouwen met overgewicht of matige obesitas, zowel in de gevaste als in de postprandiale fase.

Hoofdstuk 5 en 6 beschrijven de effecten van de inname van een karnemelkdrank waaraan luteïne-verrijkte eidooier werd toegevoegd op cardiovasculaire gezondheidsparameters. Honderd-en-een mannen en vrouwen, die begintekenen van leeftijd gerelateerde maculadegeneratie (LMD) hadden, namen deel aan deze studie. Hen werd gevraagd het drankje, gedurende één jaar, dagelijks in te nemen. Voor deze populatie was gekozen, omdat het primaire doel van de studie was om de effecten van consumptie van de luteïne-verrijkte eidrank op plasma luteïneconcentratie en visuele functie te bepalen. De secundaire doelstelling was het bepalen van de effecten van lange-termijn consumptie van dit drankje, met daarin anderhalve eidooier, op cardiovasculaire risicofactoren. De luteïne-verrijkte eidrank verhoogde de plasma luteïneconcentratie met 83\%. Er werden geen significante effecten op serum totaal-, HDL- en LDL cholesterolconcentraties gevonden. Subgroep analyses lieten zien dat deelnemers die op basis van serum campesterol:lathosterol ratio waren geclassificeerd als 'cholesterol absorber' vooral voordeel zouden kunnen hebben van de luteïneverrijkte karnemelkdrank, omdat HDL cholesterol en luteïneconcentraties in deze groep, waarin de luteïne-verrijkte eidrank werd geconsumeerd, duidelijker stegen dan in de groepen met een gemiddelde of lage ratio. De consumptie van de luteïne-verrijkte ei/karnemelkdrank had net als voor de resveratrol interventie geen effect op het 
postprandiale- glucose en lipidenmetabolisme of vaatwandfunctie gemeten door middel van FMD.

Kortom, de studies die in deze thesis staan beschreven, waren opgezet om het effect van voedingsinterventies op cardiovasculaire risicomarkers te bepalen. De hypothese was dat trans-resveratrol een positief effect zou hebben op deze markers, terwijl werd verwacht dat de inname van de luteïne-verrijkte cholesterol-rijke eidrank voor één jaar een negatieve invloed zou hebben op cardiovasculaire risicomarkers. Er werd echter geen bewijs gevonden voor een positief effect van trans-resveratrol op metabole risicomarkers, terwijl geen negatief effect op deze markers werd gevonden na de één jaar durende consumptie van de luteïne-verrijkte ei/karnemelkdrank. 
Valorisation 


\section{Valorisation}

Besides the scientific value of the research presented in this thesis, the results can also be described in terms of societal and economic value, also known as 'valorisation'. The National Valorisation Commission has defined valorisation as 'the process of creating value from knowledge by making knowledge suitable and/or available for economic and/or societal use and translating that knowledge into competitive products, services, processes and entrepreneurial activity' (202).

Finding effective strategies for preventing cardiovascular disease (CVD) and the metabolic syndrome (MetS) might lead to healthy ageing and an improved quality of life in later stages of life. This thesis studied the effect of trans-resveratrol and luteinenriched eggs on several markers of CVD and the MetS, such as lipid and lipoprotein metabolism, endothelial function and inflammation. This was studied both in the fasted state as well as postprandially. Here, the valorisation potential of these studies in terms of economic and societal relevance, and implication for specific target groups will be described.

\section{Relevance}

The studies described in this thesis contribute to the totality of knowledge evaluating the possible role of lutein and zeaxanthin in the prevention of age-related macular degeneration (AMD) and trans-resveratrol as a compound in the prevention of CVD and the MetS.

The relative risk for developing CVD is doubled in individuals diagnosed with the MetS, compared to subjects without the MetS (7). On itself, CVD is the most common cause of mortality in high-income countries $(10,11)$ and accounts for $35 \%$ of all deaths in Europe (203). Therefore, preventing the development of MetS into CVD or even decreasing its prevalence is an obvious target to control health care costs and improve quality of life. Earlier research indicated that trans-resveratrol could potentially help preventing the MetS.

AMD is the most common cause of blindness in developed countries and accounts for $8.7 \%$ of the blindness worldwide. It is particularly present in individuals $>60$ years of age. Therefore, the number of people affected with AMD is worldwide expected to grow from 196 million people in 2020 to 288 million in 2040 (204). This clearly shows the need for products that prevent or treat AMD. The lutein enriched egg yolk containing dairy drink that was given to subjects in two of the papers in this thesis aimed to prevent AMD progression. However, when consuming the lutein-enriched egg drink would lead to an increased risk for CVD through an increased intake of dietary cholesterol, this would be a major drawback. 


\section{Target groups, activities and products}

Providing increased amounts of the carotenoids lutein and zeaxanthin through the combination of a lutein-enriched egg-yolk incorporated in buttermilk was primarily developed as a tool to deliver the carotenoids within a matrix (egg-yolk) that would enhance the bioavailability of both compounds. By this approach, delivering lutein and zeaxanthin to the macular region in the retina would be enhanced, resulting in increased macular pigment, which in turn could help preventing or slowing down the progression of macular degeneration. This thesis describes that lutein and zeaxanthin concentrations in plasma were significantly increased after 1-y consumption of the lutein-enriched egg drink, whereas no increase was observed in the group that consumed the control drink. An additional paper (van der Made et al., submitted), which was outside the scope of this thesis, describes the positive effects of consuming the egg-drink on parameters for visual function in subjects who had early signs of age-related macular degeneration. The combination of these results show that the use of the lutein-enriched egg drink might be beneficial for individuals that have early signs of macular degeneration, without causing adverse effects on markers for cardiovascular disease such as serum cholesterol concentrations and flow-mediated dilation. However, these findings should be confirmed in additional studies. This additional research, amongst others in combination with the research presented in this thesis, could be used to complete a dossier that could be used to apply for a European Food and Safety (EFSA)-approved health claim regarding the relation between the intake of lutein enriched products and AMD risk. Eventually, when approved, this health claim can be communicated to consumers, for instance via the package of the product, but also through advertisements, articles in news papers and journals that aim to reach the target group of individuals that have an increased risk for AMD.

Results from cell, animal and human studies showed that trans-resveratrol might be a very promising agent in lowering the risk for CVD and MetS-related risk markers. However, in our studies we did not find an effect of this compound on any of the CVD related parameters that were measured. Still, our results increase the knowledge that is already present about the effects of trans-resveratrol in this specific target group. As the study was carried out in a specific target group -subjects had to be overweight or slightly obese and had low serum HDL cholesterol concentrations-, it is hard to extrapolate our results to a less specific target group. Other groups did show an effect of transresveratrol on markers for CVD, such as flow-mediated dilation (98), which indicates that more research is needed to unravel the reason for the discrepancy between the results and establish the potential of this compound in several target groups.

Although our study evaluating the effects of trans-resveratrol did not result in statistically significant changes, it widens the present knowledge regarding the effect of trans-resveratrol on CVD outcomes. Additionally, so-called 'negative' results are often undervalued, while these results are equally important besides all the positive results 
that are presented in high-ranked scientific journals regarding the advancement of scientific knowledge and translating this knowledge into the right advice for specific target groups. Therefore, the social relevance of the research into trans-resveratrol can be found in the publication of these 'negative' results, which help in generating the right advice for a specific target group.

The relevance of functional foods or food supplements in general in promoting health can primarily be found in the prevention or retention of progression of disorders such as CVD and the MetS. When an individual has a healthy lifestyle, is not genetically predisposed to a disease or disorder and follows the general dietary guidelines, such as the Dutch Guidelines for Healthy Nutrition (Richtlijnen Goede Voeding) drawn up by the Dutch Health Council (Gezondheidsraad), there is most likely no additional benefit of using functional foods or food supplements. However, when an individual does not completely succeed in following these dietary guidelines, functional foods can be helpful. Furthermore, it is impossible to gather scientific evidence for an improvement in health through the consumption of functional foods or food supplements in people that are already in perfect health. Therefore, use of these compounds should be promoted in individuals that are at increased risk, such as individuals that have only on or two out of five of the MetS characteristics or individuals that are genetically predisposed, and thus have a higher risk to suffer from CVD or MetS in later stages of life.

Risks that are associated with the use of functional foods and supplements should also be kept in mind. For instance, increased intake of antioxidants such as $\beta$-carotene or vitamin $\mathrm{E}$ or $\mathrm{C}$ can lead to a physiologic imbalance, which might result in pro-oxidant properties of these compounds. Furthermore, advice on the use of specific functional foods or food supplements should be given after extensive evaluation of these products in the context of that particular condition. This evaluation should make clear what the exact benefits in terms of health are when a (specific) population starts using a product. This benefit can be described in terms of incidence reduction of a disease or even reduction of number or deaths. Besides this, when the use of a functional food or food supplement leads to an improved quality of life or an improved performance, the benefit of using this product can be made clear. Only when results are confirmed in several independent scientific studies, these results can be considered univocal. At the moment, consumers are flooded with well-intentioned advice regarding healthy nutrition, which leads to misunderstanding and a negative impact on nutritional science. The credibility of nutritional science is under discussion for a while now; consumers do not know what and who to believe. Therefore, agencies such as the 'Voedingscentrum' in the Netherlands are very valuable in translating nutritional science, as provided through the Guidelines for Healthy Nutrition. In addition, the work done by the European Food and Safety Authority (EFSA) in providing scientific advice and opinions on the use of all kinds 
of foods and food components is highly valuable. Although translation of granted health claims to the consumer can be challenging, EFSA protects consumers from an uncontrolled proliferation of scientifically unfunded health-related slogans on nutritional products.

The future for preventing the MetS or CVD most likely comes from personalized nutrition, which considers each individuals' own response. Within a few years, personalised nutrition will become more and more regular, which will lead to specific supply to and use of functional foods in target groups that will definitely benefit from an intervention.

Finally, interaction and cooperation between academia and industry are crucial for valorisation of results extracted from scientific research. When these groups closely work together, results can easier be translated into products that lead to an improvement in quality of life and thus have societal impact.

\section{References}

1. van Drooge L, Vandeberg R, Zuijdam F, Mostert B, van der Meulen B, Bruins E. Valuable: Indicators for Valorisation. The Hague: Rathenau Institute; 2013.

2. Grundy SM. Drug therapy of the metabolic syndrome: minimizing the emerging crisis in polypharmacy. Nat Rev Drug Discov. 2006;5:295-309.

3. Go AS, Mozaffarian D, Roger VL, Benjamin EJ, Berry JD, Blaha MJ, Dai S, Ford ES, Fox CS, Franco S, et al. Heart disease and stroke statistics--2014 update: a report from the American Heart Association. Circulation. 2014;129:e28-e292.

4. Lozano R, Naghavi M, Foreman K, Lim S, Shibuya K, Aboyans V, Abraham J, Adair T, Aggarwal $R$, Ahn SY, et al. Global and regional mortality from 235 causes of death for 20 age groups in 1990 and 2010: a systematic analysis for the Global Burden of Disease Study 2010. Lancet. 2012;380:2095-128.

5. Organization WH. The European health report 2012: charting the way to well-being. Copenhagen: World Health Organization Regional Office for Europe; 2014.

6. Wong WL, Su X, Li X, Cheung CM, Klein R, Cheng CY, Wong TY. Global prevalence of agerelated macular degeneration and disease burden projection for 2020 and 2040: a systematic review and meta-analysis. Lancet Glob Health. 2014;2:e106-16.

7. Wong RH, Berry NM, Coates AM, Buckley JD, Bryan J, Kunz I, Howe PR. Chronic resveratrol consumption improves brachial flow-mediated dilatation in healthy obese adults. J Hypertens. 2013;31:1819-27. 

Dankwoord 
Daar is ie dan, het 'boekje'! Wat ben ik blij dat ik deze woorden eindelijk mag schrijven! $\mathrm{Nu}$ is de tijd dan ook écht aangebroken om alle mensen te bedanken die me de afgelopen jaren op wat voor manier dan ook hebben geholpen, want alleen had ik dit natuurlijk nooit voor elkaar gekregen. Daar gaat ie; het meest gelezen hoofdstuk van het proefschrift $: ;$

Als eerste wil ik mijn promotoren bedanken. Beste Jogchum, bedankt voor je eindeloze optimisme en je verfrissende inzichten. Als ik even niet meer wist waar ik de motivatie moest vinden, dan had jij altijd de juiste woorden om weer verder te gaan. Ook bedankt voor de leerzame en gezellige momenten tijdens congresbezoeken! Beste Ronald, enorm bedankt voor alles wat ik van je heb mogen leren in de afgelopen jaren, zowel op persoonlijk als op wetenschappelijk vlak. Je snelle reactie op vragen in de 'afrondende fase' van het schrijven van dit proefschrift heb ik erg gewaardeerd. Verder moet ik eerlijk bekennen dat ik de voetbal updates en de interesse in 'de trots van het voetballand' op de maandagmorgen wel mis.

De leden van de beoordelingscommissie, Prof. dr. P. Schrauwen, Prof. dr. A. Bast, Dr. R. Draijer, Dr. J.M. Geleijnse en Prof. dr. C.G. Schalkwijk, wil ik graag bedanken voor de tijd die zij hebben gemaakt voor het lezen van dit manuscript en hun aanwezigheid tijdens de verdediging.

Zonder deelnemers zou er geen (voedings-)onderzoek mogelijk zijn. Mijn grote dank gaat dan ook uit naar de 150 mensen die bereid waren deel te nemen aan het onderzoek dat staat beschreven in dit proefschrift.

De tijd bij Humane Biologie was naast heel leerzaam ook nog eens erg gezellig. Daarvoor wil ik graag alle collega's enorm bedanken! De dagjes uit, weekendjes weg en etentjes waren meer dan de moeite waard. De collega's van de 'middenlob' wil ik graag persoonlijk bedanken. Martine, jij was samen met Pascal mijn eerste kamergenoot (toen we nog écht middenlob waren); bedankt voor het wegwijs maken in het lab en alle analyses die je hebt gedaan voor de studies die hier staan beschreven! Maud, die eer gaat natuurlijk ook naar jou; zonder al jouw werk zou dit boekje er niet zijn. Maurice, bedankt voor je belangstelling in mijn onderzoek en de moeite die je doet om de groep bij elkaar te houden tijdens de lunch (:) Yvonne en Nina, bedankt voor jullie hulp tijdens de testdagen en het bakken van die gigantische lading cakejes. De proefpersonen hebben ervan genoten ;-) En Yvonne, wat gezellig dat we weer bijna-buren zijn! Ook wil ik alle stagiaires die me hebben geholpen tijdens de testdagen bedanken. Verder wil ik Florence, Chris, Sophie, Nadine, Peter, Daisy, Herman, Els, Lotte en Charlotte bedanken voor de interesse, hulp en gezellige koffie-/theebreaks. 
Tineke, wat was het bijzonder om achter je te kunnen staan tijdens jouw verdediging! Ik heb je leren kennen als een enorm betrokken collega en waardeer je vriendschap ook enorm. Bedankt voor de berg positiviteit die je altijd met je meeneemt en voor het feit dat je altijd voor iedereen klaarstaat, ondanks je drukke leventje. Ik ben jaloers op de manier waarop jij alles met elkaar gecombineerd krijgt!

Leonie, we hebben elkaar leren kennen toen jij bijna klaar was met je promotietraject, daarna hebben we nog veel gezellige avondjes in en rond Maastricht gehad. Dat moet snel maar eens een vervolg krijgen in het Brabantse Land :;

Alle collega's bij Newtricious wil ik ontzettend bedanken voor hun interesse in (en vooral ook het geduld bij) het schrijven van dit proefschrift! Resy, Janneke, Elle, Ruud, Marieëtte, Nathalie, Dennis, Wim, Paul, Hans, Marcel en Willie; wat fijn om met jullie in dit team te mogen werken. Jos, Luc, bedankt voor de mogelijkheden die jullie me bieden om me te blijven ontwikkelen!

Lieve Sabine, bedankt voor alles; van de gezellige (en leerzame natuurlijk (;) tijd in Spanje tot het bieden van een luisterend oor wanneer nodig. Het was bijzonder om zo'n fijne vriendin als collega te hebben, wat fijn dat je vandaag achter me staat. Veel geluk samen met Ron en de kleine!

Lieve Dorien, ik kan me geen fijner kamergenootje bedenken dan jij! Bedankt voor je hulp tijdens de testdagen en het luisteren als ik weer eens iets te zeuren had. Super dat jij vandaag ook achter me staat!

Natuurlijk wil ik ook al mijn lieve vrienden en (schoon-)familie bedanken voor hun interesse en de 'afschakelmomentjes' in de afgelopen jaren. Selina en Laure, die relaxdagen in de sauna en weekendjes weg houden we erin, want wat kwamen die vaak goed van pas om alles eens even lekker te relativeren. Anouk, bedankt voor de gezellige dates in Maastricht en later in Herten en Den Bosch. Heel veel succes bij jouw verdediging! Caroline, wat fijn om een vriendin als jij te hebben, bedankt voor de lading nuchterheid die je altijd meeneemt! Lieve Alicia en Lars, ze zeggen wel eens 'beter een goede buur dan een verre vriend'; ik ben blij dat jullie beide rollen geweldig vervullen, bedankt daarvoor! Buis B is een speciale plek, Michael, Roel en Yvonne, wat een super-idee om me daar mee naartoe te vragen, bedankt voor de gezellige, relaxte tijd daar. Soms moet je een flinke berg op fietsen om te bedenken dat je met een goede duw in de rug de eindstreep ook haalt. Ook iedereen die ik niet bij naam genoemd heb; bedankt!

Ramon en Thari, jullie zijn de liefste broer en zus die ik me kan bedenken (ik hoor jullie nu denken 'dat kun je zeggen, want je hebt er van allebei maar één', maar ik meen het 
echt hoor (:)). We zijn alle drie een hele andere kant op gegaan, maar alle drie met dezelfde inzet en motivatie. Veel geluk samen met Sigrid en Joey!

Lieve pap en mam, bedankt dat jullie er altijd voor ons zijn en altijd achter al onze keuzes staan, door zo'n stabiele basis zijn we alle drie waar we nu zijn. Ook al zeggen we het niet vaak tegen elkaar: ik ben trots op ons :)

Lieve Roel, ik zei het al; Buis B is een speciale plek, want toen ineens was jij daar. Begonnen als Roel 2, ben jij nu toch echt wel mijn Roel 1. Bedankt voor je eindeloze geduld, want hoe vaak heb jij de afgelopen 1.5 jaar 'sorry, vandaag kan ik niet, ik moet aan mijn proefschrift werken' moeten horen... Om het over de rest nog maar niet te hebben ;-) We gaan er een fijne tijd van maken samen! Het is af! 
List of publications 


\section{List of publications}

van der Made SM, Kelly ER, Berendschot TT, Kijlstra A, Lutjohann D, Plat J: Consuming a buttermilk drink containing lutein-enriched egg yolk daily for 1 year increased plasma lutein but did not affect serum lipid or lipoprotein concentrations in adults with early signs of age-related macular degeneration. J Nutr 2014, 144:1370-1377.

van der Made SM, Plat J, Mensink RP. Resveratrol does not influence metabolic risk markers related to cardiovascular health in overweight and slightly obese subjects: a randomized, placebo-controlled crossover trial. PLoS ONE 2015, 10(3).

van der Made SM, Mensink RP. Dyslipidemias: Pathofysiology, Evaluation and Management - Chapter 20: Polyphenols. In: Garg A, editor. Dyslipidemias: Pathofysiology, Evaluation and Management, Denver: Springer, 2015.

Baumgartner S, Kelly ER, van der Made SM, Berendschot TT, Husche C, Lutjohann D, Plat J: The influence of consuming an egg or an egg-yolk buttermilk drink for 12 wk on serum lipids, inflammation, and liver function markers in human volunteers. Nutrition 2013, 29:1237-1244.

Schrauwen-Hinderling VB, Hesselink MK, Meex R, van der Made SM, Schar M, Lamb H, Wildberger JE, Glatz J, Snoep G, Kooi ME, Schrauwen P: Improved ejection fraction after exercise training in obesity is accompanied by reduced cardiac lipid content. J Clin Endocrinol Metab 2010, 95:1932-1938.

van der Made SM, Baumgartner S, Gonzalez-Muniesa P, Zulet MA, Martinez JA: The Use of Functional Foods in the Metabolic Syndrome. Curr Nutr Food Sci 2012, 8:25-44.

\section{Submitted manuscripts}

van der Made SM, Berendschot TT, Kijlstra A, Plat J. T Daily consumption of a buttermilk drink containing lutein-enriched egg-yolks does not affect postprandial metabolism or endothelial function. Submitted.

van der Made SM, Plat J, Mensink RP. Resveratrol does not affect markers for endothelial function and arterial flexibility in the fasting or postprandial phase: a randomized, placebo-controlled crossover trial. Submitted. 
van der Made SM, Kelly ER, Kijlstra A, Plat J, Berendschot TT. Consuming a dairy drink containing lutein-enriched egg-yolk improves macular pigment optical density and visual acuity in subjects with early AMD. Submitted 

Curriculum vitae 
Sanne Maria van der Made was born in Tilburg, The Netherlands on the 6th of January, 1986. After completing secondary school at the Theresialyceum, Tilburg in 2004, she started her study General Health Sciences, with a specialization in Bioregulation and Health, at Maastricht University, for which she obtained her bachelor's degree in 2007. In January 2009, she obtained the degree of Master of Science Physical Activity and Health after completing the European Master of Metabolism and Nutrition at Maastricht University, which included two six-month internships at the Department of Human Biology in Maastricht and the Universidad the Navarra in Pamplona, Spain. In February 2009, she started working as a research assistant at the Department of Human Biology at Maastricht University. From September 2009 on, she started her PhD research at the same department under supervision of prof. J. Plat and prof. dr. ir. R.P. Mensink. The research performed during this project is described in this thesis, entitled "Diet, postprandial metabolism and endothelial function: effects of lutein-enriched eggs and trans-resveratrol" and was partly supported by the Top Institute Food and Nutrition and "Newtricious R\&D". She was awarded with the 2nd prize in the Foppe ten Hoor Young Investigator award ceremony during the Nutritional Science Days in Deurne, The Netherlands in October 2013.

In September 2013, Sanne started working as R\&D Project Manager at Newtricious R\&D in Oirlo, The Netherlands. 